\title{
WestVirginiaUniversity
}

THE RESEARCH REPOSITORY @ WVU

Graduate Theses, Dissertations, and Problem Reports

2014

\section{Solid state formation of calcium carbide}

Patrick Egbele lyere

Follow this and additional works at: https://researchrepository.wvu.edu/etd

\section{Recommended Citation}

lyere, Patrick Egbele, "Solid state formation of calcium carbide" (2014). Graduate Theses, Dissertations, and Problem Reports. 7316.

https://researchrepository.wvu.edu/etd/7316

This Thesis is protected by copyright and/or related rights. It has been brought to you by the The Research Repository @ WVU with permission from the rights-holder(s). You are free to use this Thesis in any way that is permitted by the copyright and related rights legislation that applies to your use. For other uses you must obtain permission from the rights-holder(s) directly, unless additional rights are indicated by a Creative Commons license in the record and/ or on the work itself. This Thesis has been accepted for inclusion in WVU Graduate Theses, Dissertations, and Problem Reports collection by an authorized administrator of The Research Repository @ WVU. For more information, please contact researchrepository@mail.wvu.edu. 


\section{SOLID STATE FORMATION OF CALCIUM CARBIDE}

Patrick Egbele Iyere

Thesis submitted to the Benjamin M. Statler College of Engineering and Mineral Resources at West Virginia University in partial fulfillment of the requirements for the degree of

Master of Science

in

Mechanical Engineering

Edward M. Sabolsky, Ph.D., Chair

Ismail Celik, Ph.D.

Bruce Kang, Ph.D.

Department of Mechanical and Aerospace Engineering

Morgantown, West Virginia

2013

Keywords: Calcium Carbide, Solid-State Synthesis, Industrial Microwave Furnace Copyright 2013 lyere, Patrick Egbele 
All rights reserved

INFORMATION TO ALL USERS

The quality of this reproduction is dependent upon the quality of the copy submitted.

In the unlikely event that the author did not send a complete manuscript and there are missing pages, these will be noted. Also, if material had to be removed, a note will indicate the deletion.

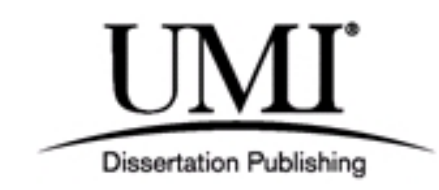

UMI 1555314

Published by ProQuest LLC (2014). Copyright in the Dissertation held by the Author.

Microform Edition () ProQuest LLC.

All rights reserved. This work is protected against unauthorized copying under Title 17, United States Code

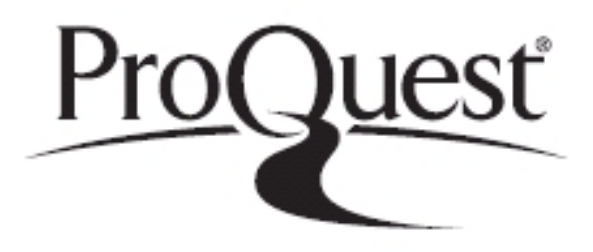

ProQuest LLC.

789 East Eisenhower Parkway

P.O. Box 1346

Ann Arbor, MI 48106 - 1346 


\section{ABSTRACT \\ SOLID-STATE FORMATION OF CALCIUM CARBIDE}

\section{Patrick Egbele Iyere}

Acetylene is known as an essential chemical building block with increasing prominent applications as a raw material in the production of important organic compounds. An indirect method used to synthesize acetylene is through the decomposition of $\mathrm{CaC}_{2}$ in the presence of water. The current work investigated a process for the synthesis of calcium carbide via a solidstate reaction route and the general kinetics of the reaction was characterized. The calcium carbide was synthesized by conventional and microwave heating $(6 \mathrm{~kW}, 2.45 \mathrm{GHz})$ of micronsized calcium oxide and carbon powders in argon. The temperature range for these reactions was between $1000-1800^{\circ} \mathrm{C}$. The work succeeded in preparing $\mathrm{CaC}_{2}$ within several minutes using microwave heating. A well-crystallized product was obtained with purities as high as $90 \% \mathrm{CaC}_{2}$. Three different phase were identified during the reactions depending upon the temperature regime; $\mathrm{CaC}_{2} \mathrm{II}\left(\right.$ at $\left.1600^{\circ} \mathrm{C}\right), \mathrm{CaC}_{2}$ II \& III $\left(\right.$ at $1650^{\circ} \mathrm{C}$ ) and $\mathrm{CaC}_{2}$ I, II \& III (at $1700^{\circ} \mathrm{C}$ ) were identified using a x-ray diffraction phase identification technique. The apparent activation energy calculated was found to be approximately $125.66 \mathrm{~kJ} \cdot \mathrm{mol}^{-1}$.

In addition to variation in reaction temperature, uniaxial pellet compaction load, reaction times, sample mass and heating rates were also varied. The influence of solid particle surface contact, carbon source reactivity and mold configuration were included in this investigation. The effects of the processing techniques and variables on the final density, microstructural properties 
and percent purity of the resulting products were characterized using the gas chromatograph, $\mathrm{X}$ ray photoelectron spectroscopy and X-ray diffraction techniques.

Finally, the calcium carbide synthesized via microwave heating yielded $80 \%$ more carbide formed compared to conventionally processed carbide at $1600^{\circ} \mathrm{C}$. The reaction kinetics was increased for all temperatures using the same feed amount. The density and degree of purity of the products were affected by reaction temperature and exposure time, starting particle size of the reactants and the dimension of the sample container. 
We approve the thesis of Patrick E. Iyere.

Date of Signature

Edward M. Sabolsky, Assistant Professor,

West Virginia University, Morgantown, WV, USA

Committee Chair

Ismail Celik, Professor,

West Virginia University, Morgantown, WV, USA

Bruce Kang, Professor,

West Virginia University, Morgantown, WV, USA 


\section{DEDICATION}

In loving memory of my paternal grandparents, Mr. \& Mrs. Mark Iyere Aisuebeogun 


\section{TABLE OF CONTENTS}

LIST OF TABLES $\quad$ V

LIST OF FIGURES vii

ACKNOWLEDGEMENTS

1.0 Introduction and Statement of the Objective 1

1.1 Goals of Research $\quad 4$

1.2 Thesis Organization $\quad 5$

2.0 BACKGROUND AND LITERATURE REVIEW 6

2.1 Electrical Resistance Furnace $\quad 6$

$\begin{array}{lr}\text { 2.2 Microwave Furnace } & 8\end{array}$

- Microwave Generation

- Microwave Transmission

- Microwave Applicator

2.3 Microwave Irradiation

- Microwave Receptive Materials

2.4 Microwave Applications in Powder Ceramic Synthesis $\quad 18$

$\begin{array}{ll}2.5 \text { Ceramic Synthesis } & 21\end{array}$

- Combustion Synthesis of Ceramics

- Carbothermal Synthesis

2.6 Calcium Carbide Investigation

- Production of calcium carbide

- Kinetics of Calcium Carbide Formation

3.1 General Experimental Description 33 
- Reaction Crucibles

- Reaction Crucibles

3.5 Experimental Procedure

- Weight Loss Measurement

- Density Measurement

- X-ray Diffraction Phase Analysis: Qualitative and Quantitative

- Scanning Electron Microscopy (SEM)

- Gas Analytical Technique

4.0 RESULTS AND DISCUSSION

4.1 Heat Energy Transfer Study

4.2 Reaction Model Discussion $\quad 52$

4.3 Carbo-thermic reduction via conventional heating technique 54

- Weight measurement and analysis

4.4 Chemical Reactions and Microwaves Heaving

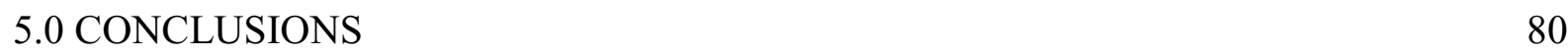

6.0 FUTURE WORK

$\begin{array}{ll}\text { APPENDICES } & 85\end{array}$

$\begin{array}{ll}\text { REFERENCES } & 87\end{array}$ 


\section{LIST OF TABLES}

Table Page

2.1 Heating elements for electrical resistance furnaces [13].

7

2.2 Dielectric constants and loss tangent of typical dielectric materials [14].

2.3: Engineering materials produced by SHS method [27].

2.4 Effect of microwave heating rate as a result of varying incident power level [31].

3.1 Characteristics of experimental starting materials.

3.2 Summary of milling parameters for 3:1 stoichiometric mixture of starting materials.

4.1 Sample pre-treatment program per run in the furnace.

4.2 Influence of particle size and applied pellets $(12.77 \mathrm{~mm}$ diameter, $2.5 \mathrm{~mm}$ thickness, $0.55 \mathrm{~g}$ average mass of powder granules) compaction load on the weight loss at reaction temperature $1500^{\circ} \mathrm{C}$ and 460 minutes reaction time in argon environment.

4.3 Weight loss as a function temperature for various carbon sources used. All experiments done at 60 minute hold time and the reacted samples placed in zirconia combustion crucibles.

4.4 Temperature study results in conventional furnace. measurements. 

completed. 


\section{LIST OF FIGURES}

Figure $\quad$ Page

1.1 Commercially important chemicals and their principal derivatives and applications 2 derived from acetylene [24].

2.1 Top and sectional view of a magnetron heating tube [14]

2.2 Electromagnetic spectrum and frequencies used in microwave processing [16].

The most used frequency for material processing is $2.45 \mathrm{GHz}$.

2.3 Relationship between the dielectric loss factor and ability to absorb microwave power for some common materials [13].

2.4 Temperature profile within the test sample in: (a) conventional heating,

(b) microwave heating and (c) microwave hybrid heating.

3.1 SEM of carbon coal obtained from WVU Coal Research Corporation.

3.2 SEM back-scattered image of 3:1 stoichiometric mixture of graphite and lime powder (Alfa Aesar).

3.3 XRD patterns of reagent grade calcium oxide (lime), graphite and the $3: 1$

stoichiometric mixture of both graphite and lime powder (Alfa Aesar).

3.4 XPS survey scan for 3:1 stoichiometric mixture of both graphite and lime powder (Alfa Aesar supplies); Stable carbon peak formed at $284.5 \mathrm{eV}$ as revealed from the detailed scans for $\mathrm{C} 1 \mathrm{~s}, \mathrm{Ca} 2 \mathrm{p}$ and $\mathrm{O} 1 \mathrm{~s}$. 
3.6 Typical temperature profile of the conventional furnace; this represent a

temperature for experiment ramped up to $1800^{\circ} \mathrm{C}$ with 60 minutes exposure time then allowed to cool down to room temperature.

3.7 Microwave Furnace Heating System; 12kW, 2.45GHz donated by Hadron Technologies Inc.

3.8 Typical temperature profile obtained during microwave heating of $\mathrm{C}-\mathrm{CaO}$ mixture and power level used.

3.9 Insulating package for microwave reaction (Note: Not drawn to scale).

3.10 Gas generation and collection apparatus (Vacuum pump is detached).

3.11 PerkinElmer ${ }^{\circledR}$ Clarus ${ }^{\circledR} 580$ Gas Chromatograph (GC) used to analyze products obtained after hydrolysis in DI water. Sample trapped in an air-tight syringes (Model: 1100 Gastight syringes, Hamilton Company, Reno, NV) and then attached to the gas sampling port on the side of the GC.

4.1 Temperature measurement profile for various sample thickness.

4.2 XRD pattern for sample (pre-treated before ball milling) with $\mathrm{Ca}(\mathrm{OH})_{2}$ impurities. $\quad 55$ Mass compositions of phases present were quantified by Rietveld technique and the calculated amount of moisture picked up is $24.29 \%$ of initial mass. The bottom parts of the graph shows the residue which is used to determine the goodness of the fit. 
furnace.

4.4 Influence of crucible configuration and particle size as a function of temperature on the weight loss of reaction completed. Graphite crucible with and without lids were used in this study.

4.5 XPS surface analysis technique depth profile scan for $1 / 3$ carbon and calcium oxide 60 mixture reacted at $1500^{\circ} \mathrm{C}$ for 60 minutes. The surface was sputtered to make sure that carbon detected is from the sample, not contaminant.

4.6 Weight loss as a function of temperature. All experiments were held at 60 and 120 minutes.

4.7 Relation between weight loss and exposure time. mixture of $\mathrm{C}$ and $\mathrm{CaO}$.

4.10: Relation between logarithm apparent reaction kinetic constants against the reciprocal of the reaction temperatures. Break-point at $1635^{\circ} \mathrm{C}$.

4.11: XRD of ball-milled lime and carbon (graphite) mixture after reaction- $1800^{\circ} \mathrm{C}$. Three $\mathrm{CaC}_{2}$ modifications identified.

4.12: SEM of ball-milled lime and carbon (graphite) mixture after reaction @ $1800^{\circ} \mathrm{C} \quad 70$

4.13: SEM of ball-milled lime and carbon (graphite) mixture after reaction @ $1700^{\circ} \mathrm{C} \quad 70$ 
4.14: SEM of ball-milled lime and carbon (graphite) mixture after reaction @ $1600^{\circ} \mathrm{C} 71$

4.15: SEM of ball-milled lime and carbon (graphite) mixture after reaction @ $1800^{\circ} \mathrm{C} 71$

4.16: Percent weight loss as a function of maximum microwave furnace temperature. $\quad 77$

4.17: Comparison of calcium carbide yields in conventional tube and microwave furnaces.

4.18: Comparison of calcium oxide consumed via microwave (MW) and conventional 78 heating. 


\section{ACKNOWLEDGEMENTS}

I would like to thank Dr. Sabolsky for his guidance, encouragement and patience throughout this research work. I would also extend my appreciation to Dr. Celik and Dr. Kang for their inputs and evaluation of my thesis.

I thank every member of our research group (present and past members) for their helpful discussions about this work, for assistance with equipment and most of all for their friendship. I would especially like to thank Dr. Steve Rowan for several helpful discussions on this work. I also thank Dr. Singh for his assistance in using the GC equipment.

I would also like to thank my family for their love and support through all of my endeavors in my life. I am especially grateful to my dad as well as Mr. Kehinde Phillips and Mrs. Michelle Ukoh, that encouraged me to reevaluate my plans after undergraduate school and attend WVU and for their all-round support.

I would also like to acknowledge LP Amina for supporting this research and Hadron Technologies for the microwave furnace donation for this work.

This technical effort was performed in support of the US-China Clean Energy Research CenterAdvanced Coal Technology Consortium's (CERC-ACTC) ongoing research in coal-to-chemical conversion processes under the DOE contract DE-PI0000017. 


\subsection{Introduction-statement of the objective}

The chemical industry is one of the largest industries in the United States and it is energy driven. The industry heavily relies upon energy inputs for raw materials processing as well as for fuel and power. In a bid to diversify the energy and chemical markets, and subsequently reduce its complete reliance on oil and natural gas, attention is once more been turned to calcium carbide, $\mathrm{CaC}_{2}$ formed from cheap carbon sources (e.g. coal, coal char, etc.), to produce acetylene which will serve as a viable, cheap and clean alternative source of energy and chemical industry feedstock, having been the mainstay in the early 1900s. World production of acetylene peaked between 1960 and 1970 [24].

Calcium carbide is regarded an economic route to acetylene production industrially; combined with water to generate acetylene according to reaction 1 below:

$$
\mathrm{CaC}_{2}(\mathrm{~s})+2 \mathrm{H}_{2} \mathrm{O}(\mathrm{l})<=\mathrm{C}_{2} \mathrm{H}_{2}(\mathrm{~g})+\mathrm{Ca}(\mathrm{OH})_{2}(\mathrm{~s})
$$

An approximately $80 \%$ of the acetylene produced using calcium carbide are used for chemical related synthesis in the United States [1].

China also relies on acetylene to a large extent as an industrial fuel, mainly because it costs less to make and use domestically than to import petroleum products for the same purpose. China's interest is based on the abundance of large deposit of both coal and lime stones resources locally, which can be used to cheaply produce calcium carbide.

Acetylene is known as an essential chemical building block with increasing prominent applications as a raw material in the production of important organic compounds such as vinyl chloride monomer, acetic acid and anhydride and 1,4 butanediol. A list of commercially 
important chemicals and their principal derivatives and applications are shown in Figure 1.1 [24].

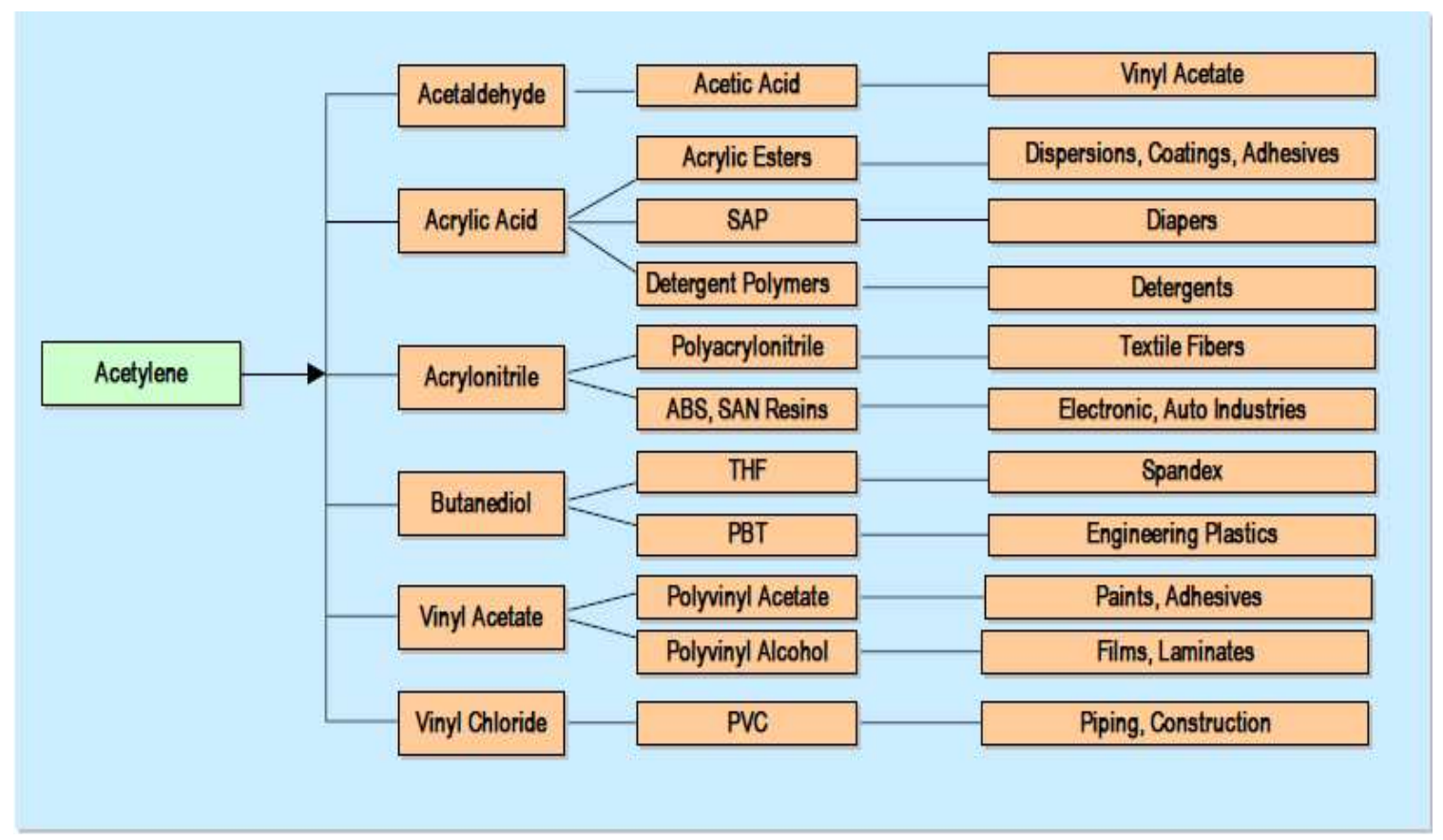

Figure 1.1: Commercially important chemicals and their principal derivatives and applications derived from acetylene [24].

Additionally, non-chemical related industrial usage of acetylene makes up about $20 \%$ in the United States: oxy-acetylene welding and metal cutting, materials heat-treatment and production of carbon for batteries. Iron and steel industry also use calcium carbide in steel desulphurization and as flux agent in steel making [1].

Calcium carbide can be prepared by carbo-thermal reduction of metal oxides, direct reaction between metal elements and carbon, and gas phase reaction of metal halides and appropriate hydrocarbons [2]. For instance, some authors have reported syntheses of $\mathrm{CaC}_{2}$ from coal, coal char, graphite and bio char as their carbon source [2-11]. However, most of the $\mathrm{CaC}_{2}$ produced 
via these processes involved long carbo-thermic reactions (which take several hours) of $\mathrm{CaO}$ and carbon source at temperatures in excess of $2000^{\circ} \mathrm{C}$ in industrial furnaces. Carbo-thermic reduction of lime is known and believed to be a very simple and economical process for the synthesis of $\mathrm{CaC}_{2}$ because of the availability of cheap raw materials used as precursors [11]; however, the high temperatures and long heating times required, mainly due to the inefficient nature of the heat transfer between the heat source and the reaction charge, makes this process a very energy-intensive synthesis route. Therefore, this process is less attractive in commercial terms, considering the power consumption requirements to run the industrial furnaces that provide the heat to cause the reaction. Consequently, there is much interest in the possible formation of calcium carbide at a lower cost of production, with less energy usage and at relatively lower temperature ranges with high yield and purity by other means [58].

Alternative synthesis routes that can provide a wide range of comparative advantages are being explored. Microwave heating seems a suitable option comparatively. The use of microwave energy has attracted significant attention in recent years as an alternative method for processing materials. In the past decades, carbide, oxide, and nitride materials have been synthesized using microwave heating techniques. One of the most important reasons for this is the potential for the reduction in manufacturing costs due to shorter processing times, such as ultra-rapid heating/cooling rates and shorter reaction times.

Microwave heating of materials as an alternative solid-state synthesis route provides faster heating and cooling rates including volumetric heating of large and small samples which in turn eliminate damage usually caused by thermal gradient commonly experienced with conventional 
methods. Microwave synthesis does offer a fast and efficient means of processing certain ceramic carbide powders; however, there is need to carefully select the ceramic system, given the inability to combine microwaves with a vacuum environment.

\subsection{Goals of Research}

The main focus of this research is to obtain experimental data and measure conversion rates for calcium carbide formation at $1000-2200^{\circ} \mathrm{C}$ to compliment and substantiate modeling work. The objectives of these exploratory works are thus:

1) Characterize the reaction rates of carbon and calcium oxide precursors with various size ratios and chemistries,

2) Develop a method to obtain kinetic reaction constants and draw a correlation between the particle characteristics and the kinetic constants, and

3) Investigate the reaction mechanism and effect on kinetic constants by utilizing microwave heating (in comparison to conventional convection heating).

In order to address these set objectives, the following tasks were required to be completed in this research:

1) Directly synthesize calcium carbide in conventional and microwave furnace by reducing effective volume to minimize vaporization.

2) Investigate the reaction and how it may change depending upon precursor characteristics and heating method.

3) Research the influence of solid-solid surface contact and mass fraction.

4) Investigate the role of morphology and microstructural differences. 
5) Investigate an alternative low cost, repeatable fabrication technique for calcium carbide formation.

\subsection{Thesis Organization}

This thesis is composed of six chapters. The first chapter is an overview of the exploratory research work: the essence of this research work; importance to the energy world and what tasks needed to be completed in order to achieve these set objectives. Chapter two provides a detailed background of refractory ceramics, heating techniques and methods, previous and current synthesis approach and materials used. The synthesis, preparation, and characterization methodology of the formed calcium carbide are covered in Chapter three. In chapter four, results obtained in the course of this work are reported and discussed. Chapter five concludes the objectives of the project completed while chapter 6 presents the future areas to continue the work. 


\subsection{Background and Literature Review}

Over the past decades, the ceramic industry has seen a rising interest in the processing and synthesis of fine particles, with high-tech ceramic materials applications been the driving force. Usually high temperatures $\left(>1200^{\circ} \mathrm{C}\right)$ are required for synthesis by both solid-state and liquidphase processes. Most of these reactions are usually completed inside furnaces which rely on heat energy from fuel combustion, electricity, induction heating etc. Laboratory and industrial scale furnaces, irrespective of sizes, are vital machine tools that serve as heat source for material processing, heat treatment or sintering in laboratory, industrial and engineering applications [12, 17]. The needed heat to cause material transformation or refinement is supplied through direct, indirect or hybrid heating of the materials or article inside a furnace compartment.

\subsection{Electrical Resistance Furnace}

Furnaces are devices use to apply heat to a material in order to cause a change in form and/or properties. Electrical resistance furnace uses the principle of direct or indirect heating. An electric resistance furnace is capable of operating at temperatures up to $2075^{\circ} \mathrm{C}$ in vacuum or in hydrogen or noble-gas atmospheres. Problems of outgassing and atmosphere contamination by furnace materials are largely circumvented by eliminating insulation [25].

In direct-heat electric furnaces, materials to be heated are used as the resistor, while the insulated enclosure of the furnace is used to retain the heat. In contrast, the heating method in a resistance furnace that uses indirect heating is such that electric current passes through the heating element, or directly through metallic material and its internal resistance opposes the flow of current to produce the needed heat. Materials with high thermal conductivity and stability, low vapor pressure at elevated temperature are used as heating elements and some examples of the ones in 
use are: silicon carbide, tin oxide, graphite, zirconia, molybdenum disilicide, etc. Table 2.1

contains relevant information on attainable useful temperatures (or temperature range) of some materials; silicon carbide being the most widely used non-oxide ceramic heating elements for high temperatures applications, but decomposes between $1500^{\circ} \mathrm{C}-1600^{\circ} \mathrm{C}$ in ambient environment.

Table 2.1: Heating elements for electrical resistance furnaces [13].

\begin{tabular}{lcl}
\hline Material & Maximum useful temperature $\left({ }^{\circ} \mathrm{C}\right)$ & Usable atmosphere \\
\hline Chromium alloys & & \\
Chromel C, Nichrome, Kanthal DT & 1100 & ONR \\
Kanthal A, Chromel A & 1300 & ONR \\
Metals & 1400 & ONR \\
Pt & $1500-1700$ & ONR \\
Pt-Rh alloys & 1800 & NR \\
Mo & 2800 & NR \\
W & & \\
Ceramics & 1500 & ON \\
SiC & 1700 & ON \\
MoS & 1800 & ONR, shock \\
Lanthanum chromite & 2000 & ONR, shock \\
Thoria, stabilized & 2800 & NR \\
Zirconia, stabilized & 3000 & \\
Graphite & & \\
\hline
\end{tabular}

${ }^{a} \mathrm{O}$, oxidizing; $\mathrm{N}$, neutral; $\mathrm{R}$, reducing. Shock, particularly poor resistance to thermal shock.

Graphite and tungsten heating elements can withstand over $3000^{\circ} \mathrm{C}$ in non-oxide environments; the tendency to contaminate the heated sample at this operating reaction temperature is very high as well. The choice of materials used as heating elements is not determined only by the expected maximum temperature but also the reaction environment the material or article to be heated will be subjected to. For reactions requiring high temperature in an oxidizing environment, elements made of zirconia are preferred. At room temperature zirconia elements do not conduct electricity. They must thus be preheated by secondary heating elements, which are an integral part of the furnace design [26]. In a reducing atmosphere, graphite, tungsten or molybdenum heating elements are much more suitable. 
The mode of heat transfer inside a resistance furnace is by convection, radiation and conduction. Heat energy generated is transferred to the material to be heated from the set of heating elements or a heating mantle of various design configurations. This mode of heat transfer to the article to be heated constitute a major challenge as experienced in heating of larger ceramic samples; it usually takes longer time to reach required temperature in the inner core as heat is transferred from the material surfaces. Thermal stress in the material caused by thermal gradients as a result of this type of heating (convection and conduction) to a ceramic bulk is also of utmost concern to researchers. Other concerns about conventional heating mechanism are the use of expensive heating elements and the high cost of energy needed to run large industrial electric furnaces over a long period of time.

\subsection{Microwave Furnace}

Microwave furnaces are electromagnetic furnaces with the capability to generate indirect heating through higher frequencies. Generally, adopted microwave frequency for large industrial production is $915 \mathrm{MHz}$. For smaller production scale, scientific and domestic purposes, the worldwide adopted microwave frequency is $2450 \mathrm{MHz}(2.45 \mathrm{GHz})$ [35]. The difference between Domestic Microwave Oven, DMO and the industrial ovens is in their power input. The maximum allowable power for domestic applications is $700 \mathrm{~W}$ while about $6 \mathrm{~kW}$ for industrial microwave oven. In contrast to the conventional furnace mode of energy transfer into the experimental sample, microwave energy generated is transferred to the material to be heated through molecular interaction with electro-magnetic field within the material. What is seen in the conventional furnace is that thermal energy is transferred into a reaction system by using electricity, gas or coal combustion to heat up the heating mantle or elements; this in turn heats up the vessel, and the vessel then heats up the reaction mixture until it attain homogenous 
temperature with a possibility of some thermal gradients [48]. This thermal energy will supply the necessary energy to the reaction mixture to cause the product formation. The mechanism of energy transfer in a microwave is however significantly different. Microwave energy heats the sample through direct activation. Instead of heating the microwave, then the vessel, energy is transferred to the reaction components within the solution, providing two different benefits:

- More efficient energy transfers to the reaction mixture, instead of the vessel.

- $\quad$ Reaction components at the center of the reaction are heated at the same rate as reactants near the walls of the vessel [48].

This energy is much more precise and can be controlled to a specific region in a material. Microwave heating can be used to form new materials by initiating chemical reactions through selective heating of materials. The three major components of a microwave furnace are: the source, the transmission lines and the applicator [14].

\subsubsection{Microwave Generation}

Vacuum tubes are mostly used as the electromagnetic source considering the required high power generation and frequencies for microwave heating. The most widely used microwave source for heating is the magnetron because of it cost effectiveness and compactness compared to linear beam tube. The magnetron is basically a high power microwave oscillator which converts high voltage DC electrical power into microwave radiation. The theory of operation is such that potential energy of an electron cloud near the cathode is converted into radiofrequency energy in a series of cavity resonators. Figure 2.1 shows the working parts of a typical domestic microwave oven magnetron. 

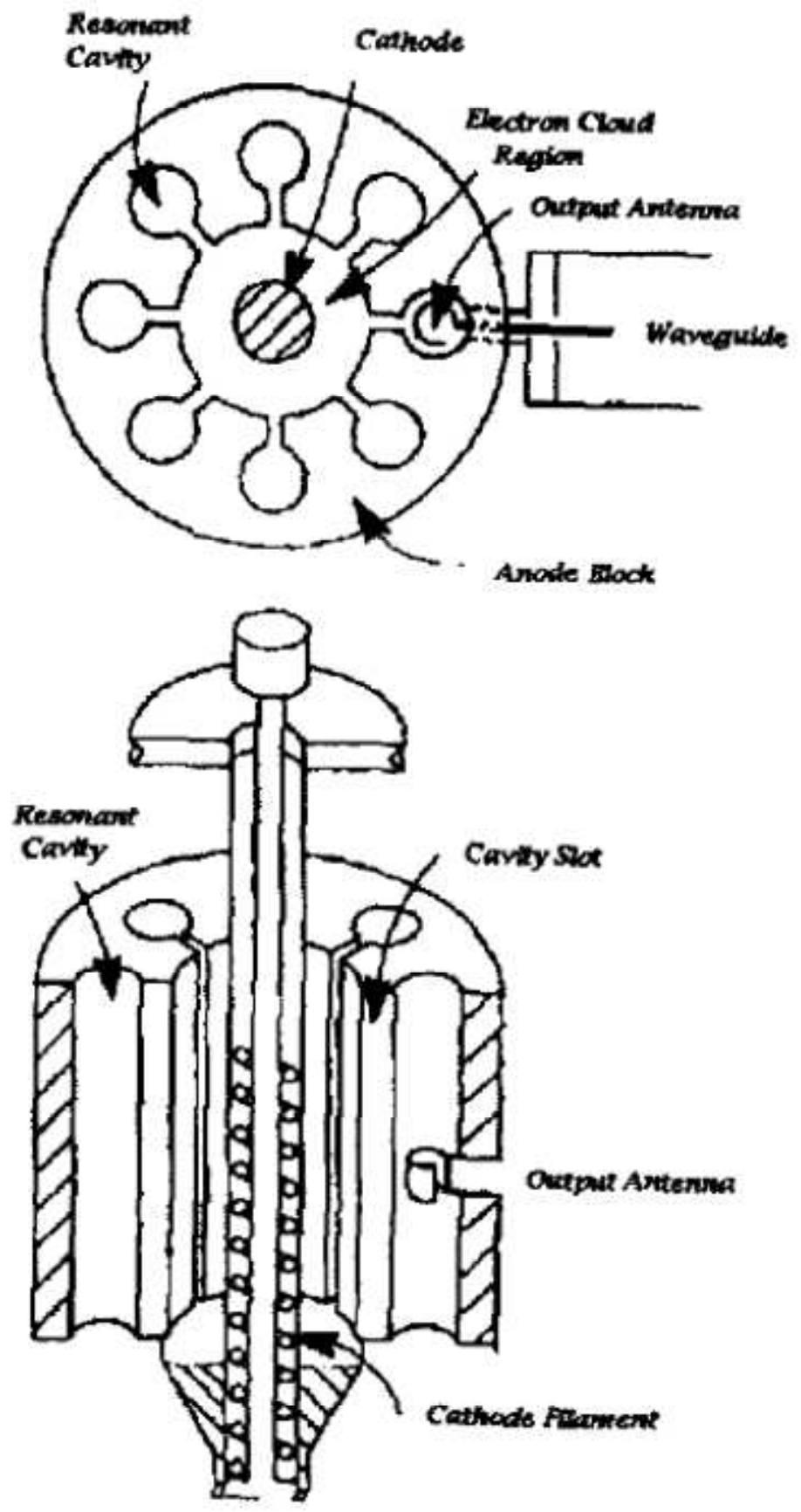

Figure 2.1: Top and sectional view of a magnetron heating tube [14].

Characteristically, magnetrons heating source operates at about 60 to $65 \%$ efficiency. The output power of the magnetron can be controlled by adjusting the period of operation or adjusting the cathode current or magnetic field strength, and is limited by the anode temperature. The excess 
energy generated in heating the anode and cathode is removed by radiative fins (at $2.45 \mathrm{GHz}$, aircooled anodes can be used up to about $1.5 \mathrm{~kW}$ ) or a circulating water jacket (for higher power densities, up to $6 \mathrm{~kW}$ ) $[14,48]$. The other types of common microwave generating sources are Klystrons and Traveling wave tubes (applications are found in variable-frequency microwave furnaces).

\subsubsection{Microwave Transmission}

Generated radiation as a result of accelerated electrons has to be channeled in the direction of the material sample to be heated inside the furnace chamber of the microwave oven. The two transmission devices commonly used are determined by the frequency required. For low power (lower frequencies), coaxial cable is used extensively; for communication, whereas high power operating devices require hollow metal tubes, because of the high current density. Two modes of microwave propagation are possible in waveguides: transverse electric (TE) and transverse magnetic (TM). Other components used for protection purposes are the circulators, directional couplers and turners $[14,48]$.

\subsubsection{Microwave Applicator}

Microwave energy from the waveguide is transferred to the material sample with the aid of the applicator. The temperature fields within the material undergoing microwave heating are inherently linked to the distribution of the electric fields within the applicator. The cavity in which the material sample is laced is considered an applicator as well. There are several types of applicators, with the common ones including waveguides, traveling wave applicators, single mode cavities, and multi-mode cavities [14]. 
For processing materials, resonant applicators, such as single mode and multi-mode applicators, are the most common because of their high field strengths. The type of applicator used in a microwave processing system often depends on the materials to be processed. Commercially available single mode, multi-mode, and variable frequency multi-mode processing systems are all used for microwave processing research, and each of these systems has advantages and disadvantages. Single-mode applicators are ideal for heating small volumes; multimode applicators are used for processing larger samples, where more uniform heating is desirable. The way a sample is placed within the cavity also affects the heating efficiency and uniformity; therefore turntables are used to move the material sample through the field thereby lessen hot and cold spot effects. In addition, mode stirrers (rotating metallic blades) are also employed to obtain more uniform heating by perturbing the electrical field within the cavity [14].

\subsection{Microwave Irradiation}

A non-ionizing electromagnetic radiation with $1 \mathrm{~m}$ to $1 \mathrm{~mm}$ in wavelength corresponding to a frequency of 300 to $300 \mathrm{GHz}$ in the electromagnetic spectrum (as shown in figure 2.2) is known as microwave radiation. Although microwaves can travel at the speed of light, microwave however varies in length, based on frequency. There are four frequencies that are allowed by the government for commercial microwave use: $915 \mathrm{MHz}, 2450 \mathrm{MHz}, 5800 \mathrm{MHz}$, and 22,125 MHz. The frequency $2450 \mathrm{MHz}$ has a length of $12.2 \mathrm{~cm}$, which has an appropriate penetration depth (the distance a microwave can travel into a standard sample), for use with small samples [48]. It was the work of Professor Richard Gedye, Professor Raymond J. Giguere and George Majetich in 1986 that first detailed its application in reactions, in contrast to available conventional heating techniques $[48,49]$. 


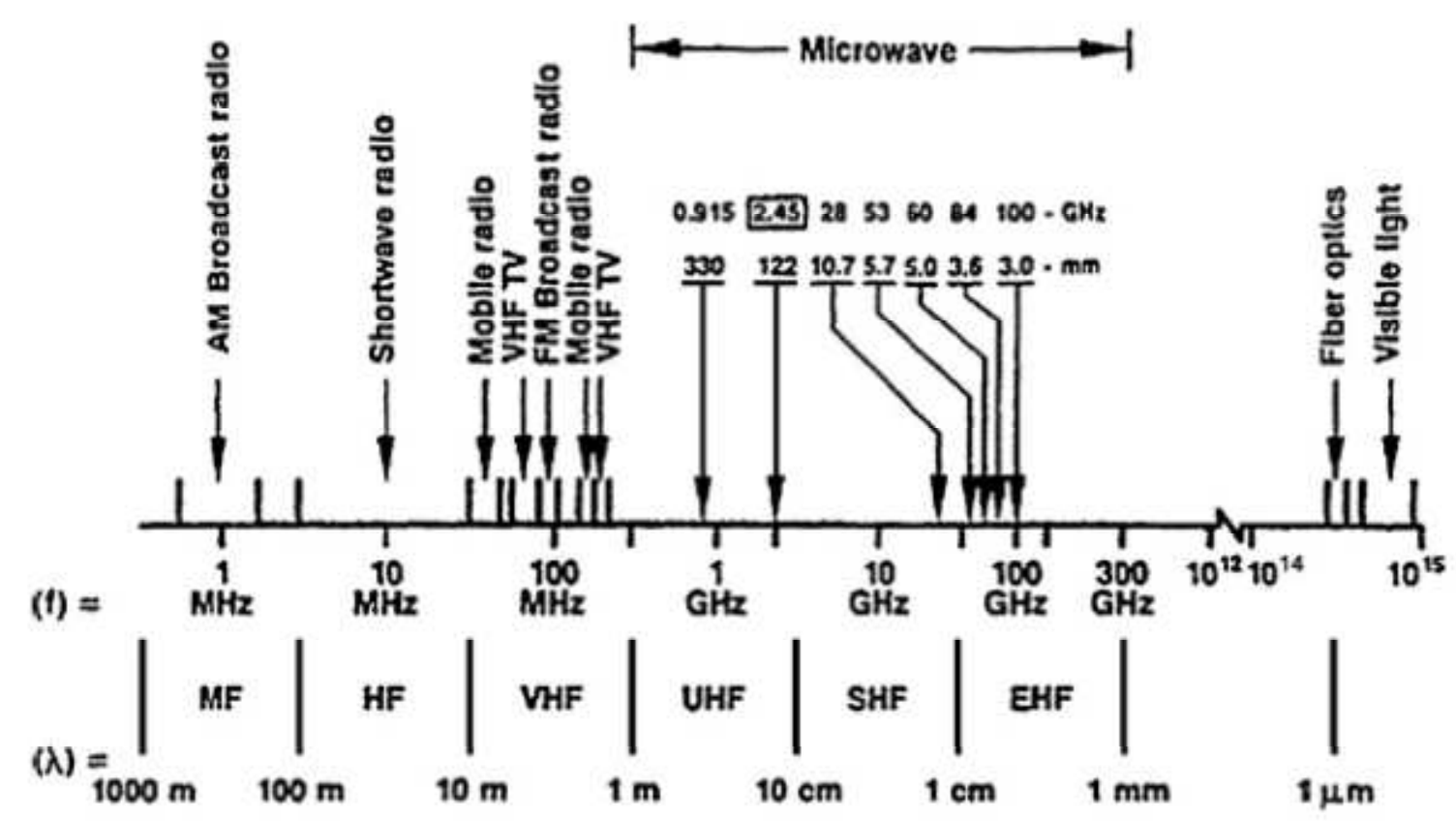

Figure 2.2: Electromagnetic spectrum and frequencies used in microwave processing [16]. The most used frequency for material processing is $2.45 \mathrm{GHz}$.

Microwave energy is a form of electromagnetic energy deposited in materials that couple with microwaves, absorb the electromagnetic energy volumetrically, and transform this energy into heat. This heating technique is not the same with the conventional heating methods whereby heat is transferred between objects by the mechanisms of conduction, radiation and conduction, from the material's surfaces, then transferred to the core of the material. In contrast, microwave heating generates heat within the material first and then heats the entire volume of the material with the elimination of temperature gradient that generally exists with the conventional approach. In comparison to x-rays, ultra violet and infrared, this form of energy does not break atomic bonding; instead it causes the bonds to rotate in a material or causes dipoles end/or ions to change their orientation/movement in phase with the electromagnetic field because of it low frequency. 


\subsubsection{Microwave Receptive Materials}

In the microwave field, materials are classified as transparent, opaque, absorbing and/or mixedabsorber based on their ability to transmit or allow an electric field through them. Both electric and magnetic properties of any material determine whether microwave radiation will be reflected, absorbed or transmitted. In figure 2.3 , the degree of susceptibility to microwave energy for various materials is shown. Microwave receptive materials experience rapid and volumetric internal heating, with reduced thermal gradient, lower processing time and relatively low energy usage due to the penetrating capabilities of the energy through the materials. This explains why the reaction kinetics and sintering of materials susceptive to microwave energy are relatively faster when compared to conventional process [15].

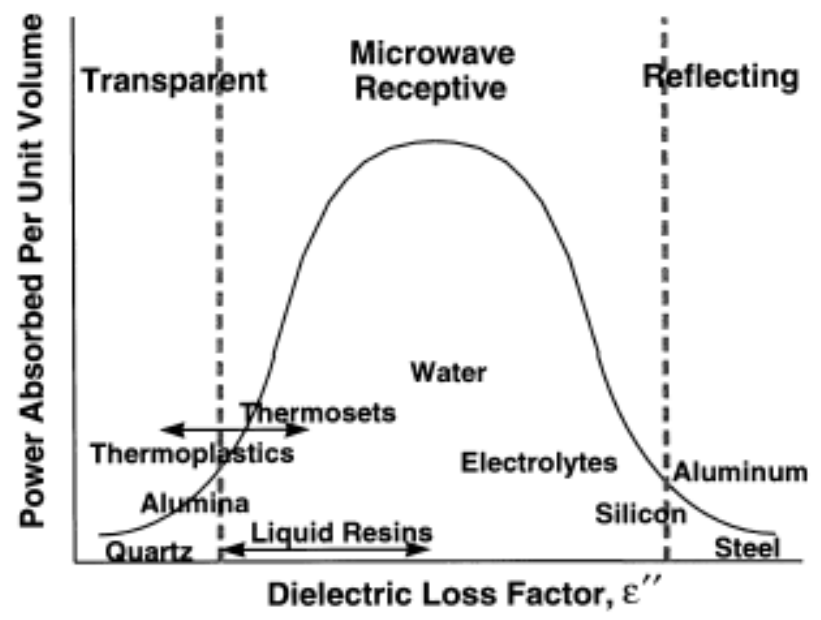

Figure 2.3: Relationship between the dielectric loss factor and ability to absorb microwave power for some common materials [13].

Other materials like many ceramics that are transparent or poor absorbers of microwave energy can be heated to a critical temperature to make them good microwave absorbers. This can be 
achieved through the addition of microwave absorbing additives or second phases in these types of ceramics.

To a large degree, the absorption capabilities of every material depend on its compositional make-up (including structure defects inherent in the material) and temperature. The absorption behavior of a material also depends on the dielectric constant and loss tangent properties of the materials (dielectric properties); these properties are related to temperature, moisture content, density and material geometry, and are useful in predicting power absorption and depth of microwave penetration characteristics of any material in a microwave energy field. The loss tangent $(\tan \delta)$ indicates the ability of a material to be polarized and heated.

The complex permittivity, $\square *$, of a dielectric determines the amount of interaction with microwave energy, through the real part $\left(\square_{\mathrm{r}}{ }^{\prime}\right)$ and the imaginary part ( $\square$ eff' $)$. The relationship between permittivity and the heating of the dielectric can be related to the power absorbed per unit volume $\left(\mathrm{W} / \mathrm{m}^{3}\right)$ through the following equations [18]:

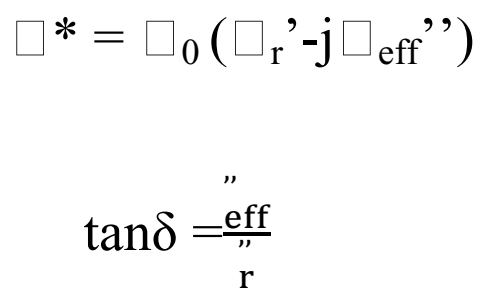

P, power absorbed per unit volume $\left(\mathrm{W} / \mathrm{m}^{3}\right)=\sigma / E /^{2}=2 \pi f_{0 \mathrm{r}}{ }^{\prime} \tan \delta / E /^{2}$

Where $\mathrm{E}$ is the electric field, $\sigma$ is the electrical conductivity, $\mathrm{f}$ is the microwave frequency,

$\square_{0}$ is the permittivity of free space, $\square_{\mathrm{r}}$ ' is the relative dielectric constant, $\square_{\text {eff }}$ ' is the dielectric loss, $\mathrm{j}=(-1)^{1 / 2}$ and $\tan \delta$ is the loss tangent. 
The penetration depth at one-half power, $\mathrm{D}$, is a useful parameter to determine how microwaves penetrate and propagate through an absorbing material (18):

$$
D=\frac{3 \lambda_{0}}{8.686 \pi(\tan \delta)\left(\varepsilon_{\mathrm{r}}^{\prime} / \varepsilon_{0}\right)^{1 / 2}}
$$

Where $\lambda_{0}$ is the incident wavelength, $\varepsilon_{0}$ is the permittivity of free space, $\varepsilon_{\mathrm{r}}{ }^{\prime}$ is the relative dielectric constant and $\tan \delta$ is the loss tangent.

When microwave energy heats a material, the rate of temperature increase in the material can be derived as follows [51]:

$$
\frac{D T}{d t}=\frac{P}{\rho C_{p}}
$$

Where $\mathrm{C}_{\mathrm{p}}$ is the specific heat of the material and $\rho$ is the density of the material.

In table 2.2, the dielectric properties (dielectric constant and loss tangent values) for some materials (highly absorptive materials (with $\tan \delta \geq 10^{-1}$ )); dielectric constant, $\dot{\varepsilon}_{\mathrm{r}}$ that measures the ability of the material to be electrically polarized by an applied electric field, are presented.

Other vital influences that control diffusion behavior in microwave receptive materials are the synthesis environment and additives inclusion. Materials that show high conduction and no dielectric properties are regarded as reflectors since they inhibit the propagation of electromagnetic waves by reflection. Transparent materials are low dielectric loss materials allowing for the passage of microwaves without any losses. Microwave absorbing materials have high loss and absorb microwaves energy depending on dielectric loss factor. Fine ceramic powder such as silicon carbide, zirconia carbide, aluminum nitride, magnesia, carbon and 
vanadium oxide, have high loss tangent and as such, have the potential to couple with microwaves at room temperature. While, low-loss materials such as alumina, titanium oxide,

Table 2.2: Dielectric constants and loss tangent of typical dielectric materials [14]

\begin{tabular}{lcc}
\hline \multicolumn{1}{c}{ Material } & \multicolumn{1}{c}{$\varepsilon_{r}^{\prime}$} & $\tan \delta$ \\
\hline Air & 1.0006 & \\
Ethyl alcohol & 25 & 0.1 \\
Aluminum oxide & 8.8 & $6 \times 10^{-4}$ \\
Bakelite & 4.74 & $22 \times 10^{-3}$ \\
Carbon dioxide & 1.001 & \\
Germanium & 16 & \\
Glass & $4-7$ & $1 \times 10^{-3}$ \\
Ice & 4.2 & 0.1 \\
Mica & 5.4 & $6 \times 10^{-4}$ \\
& & \\
Quartz (fused) & 3.8 & $7.5 \times 10^{-4}$ \\
Rubber & $2.5-3$ & $2 \times 10^{-3}$ \\
Silica (fused) & 3.8 & $7.5 \times 10^{-4}$ \\
Silicon & 11.8 & \\
Snow & 3.3 & 0.5 \\
Sodium chloride & 5.9 & $1 \times 10^{-4}$ \\
Soil (dry) & 2.8 & $7 \times 10^{-2}$ \\
Styrofoam & 1.03 & $1 \times 10^{-4}$ \\
Teflon & 2.1 & $3 \times 10^{-4}$ \\
Titanium dioxide & 100 & $15 \times 10^{-4}$ \\
Water (distilled) & 80 & $4 \times 10^{-2}$ \\
Water (sea) & 81 & 4.64 \\
Wood (dry) & $1.5-4$ & $1 \times 10^{-2}$ \\
\hline
\end{tabular}

silicon nitride and zirconia require preheating beyond a critical temperature before coupling with microwave energy. 
Above a critical temperature, many materials experience an increase in their susceptibility as the dielectric loss factor increases, microwave couple well with the material and this simply implies that the heated materials also contribute additional heat during synthesis process [11, 18].

The use of some materials as subsectors (preheaters) during microwave heating depends on the response rate of the material in absorbing microwave energy; this is especially useful to aid reduction of heating rate and uniformity of sintering for ceramic insulators in most instances. These susceptor materials are usually in coarse powder sand but they could actually be in solid block as well, for example a rod like shape, for strategic placement in preferred position inside the heating package. High dielectric loss materials such as silicon carbide with a high response rate are most times used as sintering bed or packaging material for a ceramic insulator that only absorbs microwave energy at elevated temperature. The configuration and arrangement of these suceptor to be used per specific ceramic powder are important factors. For instance, a density as high as $99 \%$ can be achieved for a zirconia specimen at $1100^{\circ} \mathrm{C}$ sintering temperature $[19,20]$.

\subsection{Microwave Applications in Powder Ceramic Synthesis}

Sutton [18] first gave an insight into microwave applicability in ceramics processing in 1975, but experimental research on processing ceramics using microwave heating actually commenced in mid-1960. Ever since, researchers' interests especially in high temperature applications have been on the rise. Development of microwave heating technology thus commenced in the middle to late period of the 1980 's, its advantage over existing conventional heating methods which is characterized by fast densification and processing time for ceramic materials such as oxides, mixed oxides, non-oxides, composite ceramics, etc. [30] continued to draw researchers' interests. The heating mechanism fundamentally distinguished both the microwave and available 
conventional methods. Figure 2.4 compares the temperature profile found in three different modification of heating mechanism. In figure $2.4 \mathrm{a}$, heat transfer mode is by radiation, conduction and convection, typical conventional heating technique; in figure $2.4 \mathrm{~b}$, the sample absorbs electromagnetic energy and generates heat from inside, and in figure $2.4 \mathrm{c}$, a hybrid heating mechanism that combines both microwave heating coupled with an external heating source.
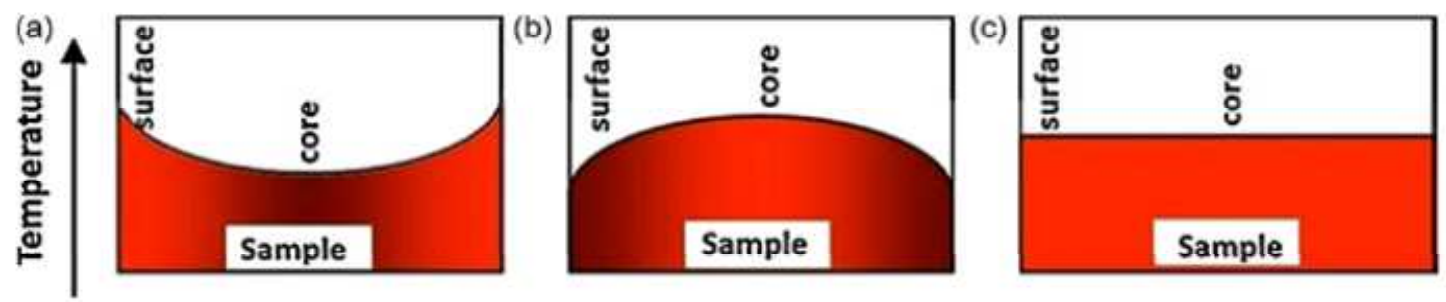

Figure 2.4 Temperature profile within the test sample in: (a) conventional heating, (b) microwave heating and (c) microwave hybrid heating [16].

Microwave processing of materials consume much lower energy to reach a higher heating rate thus reduce synthesis duration. One of the earliest data collections geared towards process parameter predictability was carried out by investigating the effect of power level on microwave heating characteristic on several materials [31] and tabulated as seen in table 2.4. Heating rate was reported to increase as the power level varied $(500-2000 \mathrm{~W})$ for high lossy materials such as $\mathrm{PbS}$ and $\mathrm{Fe}_{3} \mathrm{O}_{4}$, but did not heat very well for very low-loss materials such as $\mathrm{SiO}_{2}$ and $\mathrm{CaCO}_{3}$. In addition, the thermal runaway effect was reported for the first time. The test was run in a controlled environment and test samples used were in powder forms. The maximum attainable temperature and the time required were reported as well. Special cases observed during these tests where the maximum temperature were obtained indicated time interval, termination of experiment when sample melted, termination of the experiment when sample arced, also when 
temperature exceeded thermocouple limits, when sample not run in rapid heating rate at lower temperature, when sample not run as a result of violent boiling, and at sample boiling were represented as a, b, c, d, e, f, g and h (alphabets in superscript) as used in the table [31].

Table 2.4: Effect of microwave heating rate as a result of varying incident power level [31].

\begin{tabular}{|c|c|c|c|c|c|c|c|c|c|c|}
\hline \multirow[b]{2}{*}{ Chemical } & \multirow{2}{*}{$\begin{array}{c}\text { Wt, } \\
g\end{array}$} & \multirow{2}{*}{$\begin{array}{c}\text { Vol, } \\
\text { mLl }\end{array}$} & \multicolumn{2}{|c|}{$500 \mathrm{H}$} & \multicolumn{2}{|c|}{$1,000 \mathrm{H}$} & \multicolumn{2}{|c|}{$1,500 \mathrm{~W}$} & \multicolumn{2}{|c|}{$2,000 \mathrm{~K}$} \\
\hline & & & $\begin{array}{l}\text { Temp, } \\
{ }^{\circ} \mathrm{C}\end{array}$ & $\begin{array}{c}\text { Time, } \\
\text { min }\end{array}$ & $\begin{array}{l}\text { Terap, } \\
{ }^{\circ} \mathrm{C}\end{array}$ & $\begin{array}{l}\text { Time, } \\
\text { min }\end{array}$ & $\begin{array}{l}\text { Temp, } \\
{ }^{\circ} \mathrm{C}\end{array}$ & $\begin{array}{c}\text { Time, } \\
\text { min }\end{array}$ & $\begin{array}{l}\text { Temp, } \\
{ }^{\circ} \mathrm{C}\end{array}$ & $\begin{array}{c}\text { Pime, } \\
\text { min }\end{array}$ \\
\hline$\alpha-\mathrm{A}_{2} \mathrm{O}_{3}$ & 15.0 & 14 & 385 & 7 & 463 & 7 & 498 & 7 & 547 & 7 \\
\hline $\mathrm{CaCl}_{2}$ & 17.0 & 14 & 35 & 7 & 43 & 7 & 53 & 7 & 58 & 7 \\
\hline $\mathrm{CaCO}_{3}$ & 10.0 & 14 & 32 & 7 & 42 & 7 & 51 & 7 & 65 & 7 \\
\hline $\mathrm{CuCl}^{\circ}$ & 25,0 & 12 & 425 & $b_{3}$ & 400 & $b_{1}$ & 400 & b. 75 & 462 & b. 5 \\
\hline CuFes $_{2}$ & 25.0 & 13 & 908 & $c .75$ & 930 & d.333 & $>1,150$ & e. 25 & $>1,200$ & e. 133 \\
\hline Cuo & 25.0 & 8 & 1,083 & $b_{1} .5$ & 1,088 & b. 25 & 1,088 & b. 5 & $>1,200$ & e.25 \\
\hline $\mathrm{Cr}_{2} \mathrm{O}_{3}$ & 20.0 & 13 & 84 & 7 & $>1,200$ & $b_{4}$ & $>1,250$ & $b_{2} .5$ & $>1,200$ & $b_{2}$ \\
\hline $\mathrm{FeC} \times{ }_{2} \mathrm{O}_{4}$ & 30.0 & 12 & 110 & 7 & $>1,200$ & $e 6.25$ & $>1,200$ & e3.25 & $>1,200$ & $\mathbf{e}_{2}$ \\
\hline $\mathrm{Fe}_{2} \mathrm{O}_{3}$ & 12.0 & 14 & 60 & 7 & 87 & 7 & 101 & 7 & 130 & 7 \\
\hline $\mathrm{Fe}_{3} \mathrm{O}_{4}$ & 25.0 & 9 & 1,118 & 7 & 1,144 & $e_{3.5}$ & 1,123 & $d_{3}$ & 905 & $d_{1}$ \\
\hline $\mathrm{PeS}_{2}$ & 20.0 & 12 & 698 & 7 & 808 & 7 & 700 & d. 75 & 375 & d.17 \\
\hline $\mathrm{FeSO}_{4} \cdot 7 \mathrm{H}_{2} \mathrm{O}$ & 12.0 & 13 & 109 & 7 & 198 & $c_{5} .5$ & 118 & $c_{3}$ & 123 & $c_{1.75}$ \\
\hline $\mathrm{PbS}$ & 50.0 & 11 & 984 & d. 5 & 992 & d. 4 & $\mathbf{f}_{-}$ & & $f_{-}$ & \\
\hline $\mathrm{SiO}_{2}$ & 25.0 & 14 & 33 & 7 & 44 & 7 & 55 & 7 & 73 & 7 \\
\hline $\mathrm{TiO}_{2}$ & 10.0 & 13 & 67 & 7 & 109 & 7 & 150 & 7 & 228 & 7 \\
\hline $\mathrm{ZnCl}_{2}$ & 14.0 & $9_{9}$ & 730 & $h_{5}$ & 723 & $h_{1} .25$ & 730 & h. .25 & 593 & d. 5 \\
\hline ano & 9.5 & 14 & 87 & 7 & 128 & 7 & 184 & 7 & 301 & $c_{5} .75$ \\
\hline ZnS & 30.0 & 12 & 139 & 7 & 236 & 7 & $>1,200$ & $e_{6}$ & $>1,200$ & $e 3,75$ \\
\hline
\end{tabular}

Microwave synthesis also intensifies diffusion process leading to better grain distribution which is responsible for higher density and other improved mechanical properties observed in most high-lossy materials processed.

When processing ceramics, it is important to regulate the heating rate of the impacting energy in order to achieve desire properties. This will help prevent in-built thermal stresses that may lead to an undesired sample failure or crack during or after processing. For ceramic materials that required preheating because of their low microwave energy absorbability, the practice from literature is to control the uniformity of the ceramic microstructure which depends on the rate of 
heating, as non-uniform microwave heating could result in thermal runaway effect which could fracture the material as well. This behavior is seen in ceramics that require preheating to a critical temperature before microwave coupling due to their low loss factors. What is experienced is that a local volume reaches the critical temperature before the rest of the material bulk; that area tends to exhibit an increase in temperature as a result of this localized rapid heating and possible thermal runaway. One means of avoiding this behavior is to heat up the ceramic just before the critical temperature through a conventional heat transfer process.

Microwave processed samples are expected to yield higher densification, finer grains, phase formation, uniform microstructure, better aspect ratio and improve hardness. High heating temperature influenced the attainment of these properties and significant effort to reducing heat loss through proper insulation and better configuration is required.

\subsection{Ceramic Synthesis}

Ceramics are classified as oxide and non-oxide based engineering materials. Several technologies have been developed over the past decades for making non-agglomerated, small particle sized ceramic powders. Ceramics materials have solid, liquid and vapor phase synthetic approaches. The targeted crystal structure, material properties, process parameters and conditions influence the choice to adopt. Researchers in recent times have developed novel techniques for small sized-ceramic powder synthesis, which can be used directly to form compact bodies for variety of industrial and technological applications.

The properties of small-size structures of a material differ from that of the conventional bulk materials due to their large surface-to-volume ratios [12]. However, the unusual mechanical and electrical properties behavior experienced in some small sized-ceramics are as a result of the 
combination of a relatively grain boundary arrangement along with the interactions of electrons or dislocations. Some of the successful synthesis routes used thus far to process ceramics include combustion, mechanochemical, carbothermal and Pechini’s methods.

\subsubsection{Combustion Synthesis of Ceramics}

Combustion synthesis methods are self-sustaining highly exothermic chemical reactions that complete in minutes or seconds, for synthesizing a variety of oxide ceramics. Combustion synthesis methods are very versatile for making all sorts of advanced materials, including ceramics powder with various grain morphologies (lamellar, rod-like, etc.) [21] And several types exist: Self-propagating high temperature synthesis (SHS), low-temperature combustion synthesis (LCS), gel combustion, emulsion combustion, solution combustion synthesis (SCS), etc.

The self-propagating high-temperature synthesis (SHS) has been applied to make varieties of materials as enumerated in table 2.3. It has high rate of production, low energy usage and simple processing steps. Depending upon the expected product, small-particle sized ceramic powder can be prepared either in air or in a high pressure nitrogen environment. Two steps are involved: thorough blending of starting materials and auto-ignition. When heat is applied to a mixture of reactant powders, the activation energy for combustion is overcome, triggering a chemical reaction [50]. Powders are milled to the desired particles sizes before synthesis; their characteristics are primarily governed by heat of combustion and gas evolution. In this solid state reaction technique, the reaction is activated by heat energy [18] and this heat energy is released to cause reaction sequence among layers of reactant mixture. Desired end products can be obtained by simply manipulating the chemistry and reaction conditions. 
An example application is the improved quality obtained when aluminum nitride (AlN) ceramic powder was processed via this technique. The purity level, as reported for AlN, was raised by approximately $25 \%$, using $1-2 \mathrm{wt} \%$ urea and $\mathrm{N}_{2}$ pressure of $0.3-0.5 \mathrm{MPa}$ as optimum conditions to achieve this high product yields by the synthesis of aluminum nitride powder using aluminum containers [23].

Table 2.3: Engineering materials produced by SHS method [27].

\begin{tabular}{|c|c|}
\hline Borides & $\mathrm{CrB}, \mathrm{HfB}_{2}, \mathrm{NbB}_{2}, \mathrm{TaB}_{2}, \mathrm{TiB}_{2}, \mathrm{LaB}_{6}, \mathrm{MoB}_{2}, \mathrm{WB}_{2}, \mathrm{ZrB}_{2}, \mathrm{VB}, \mathrm{VB}_{2}, \mathrm{NiB}$ \\
\hline Carbides & $\mathrm{TiC}, \mathrm{ZrC}, \mathrm{HfC}, \mathrm{NbC}, \mathrm{SiC}, \mathrm{Cr}_{3} \mathrm{C}_{2}, \mathrm{~B}_{4} \mathrm{C}, \mathrm{WC}, \mathrm{TaC}, \mathrm{VC}, \mathrm{Mo}_{2} \mathrm{C}$ \\
\hline Carbonitides & TiC-TiN, NbC-NbN, TaC-TaN \\
\hline Cemented carbides & TiC-Ni, TiC-Mo, WC-Co, $\mathrm{Cr}_{3} \mathrm{C}_{2}$-(Ni,Mo) \\
\hline Chalcogenides & $\mathrm{MoS}_{2}, \mathrm{TaSe}_{2}, \mathrm{NbS}_{2}, \mathrm{WSe}_{2}, \mathrm{MoSe}_{2}, \mathrm{MgS}$ \\
\hline Composites & $\mathrm{TiC}-\mathrm{TiB}_{2}, \mathrm{TiB}_{2}-\mathrm{Al}_{2} \mathrm{O}_{3}, \mathrm{~B}_{4} \mathrm{C}-\mathrm{Al}_{2} \mathrm{O}_{3}, \mathrm{TiN}-\mathrm{Al}_{2} \mathrm{O}_{3}, \mathrm{TiC}-\mathrm{Al}_{2} \mathrm{O}_{3}, \mathrm{MoSi}_{2}-\mathrm{Al}_{2} \mathrm{O}_{3}$ \\
\hline Hydrides & $\mathrm{TiH}_{2}, \mathrm{ZrH}_{2}, \mathrm{NbH}_{2}$ \\
\hline Intermetallics & $\mathrm{NiAl}, \mathrm{NiAl}_{3}, \mathrm{FeAl}, \mathrm{NbGe}, \mathrm{TiNi}, \mathrm{CoTi}, \mathrm{CuAl}$ \\
\hline Nitrides & $\mathrm{TiN}, \mathrm{ZrN}, \mathrm{BN}, \mathrm{AIN}, \mathrm{Si}_{3} \mathrm{~N}_{4}, \mathrm{TaN}, \mathrm{NiN}$ \\
\hline Silicides & $\mathrm{MoSi}_{2}, \mathrm{TaSi}_{2}, \mathrm{Ti}_{3} \mathrm{Si}_{3}, \mathrm{TiSi}_{2}, \mathrm{ZrSi}_{2}, \mathrm{WSi}_{2}, \mathrm{NbSi}_{2}, \mathrm{~V}_{3} \mathrm{Si}$ \\
\hline Oxides & $\mathrm{ZrO}_{2}, \mathrm{YSZ}, \mathrm{MgAl}_{2} \mathrm{O}_{4}, \mathrm{LaCrO}_{3}, \mathrm{Bi}_{4} \mathrm{~V}_{4} \mathrm{O}_{11}, \mathrm{YBa}_{2} \mathrm{Cu}_{3} \mathrm{O}_{7}$ \\
\hline
\end{tabular}

\subsubsection{Carbothermal Synthesis}

Carbothermal processing involves the reduction of metal oxides using carbon. Its wide industrial applicability to the synthesis of carbides is owed to the utilization of low cost oxide raw materials for the process. The production of metal carbides from metal oxide reduction typically requires high temperature and energy input. The technique is primarily used to synthesize metal carbide powders, such as $\mathrm{TiC}$ using $\mathrm{TiO}_{2}$ as the precursor. This carbothermal process typically requires high temperature $\left(1700-2100^{\circ} \mathrm{C}\right)$ and long reaction times $(10-24 \mathrm{~h})[22,28]$. Woo et al [28] was the first to report the commercial production of homogenous and ultra-fine TiC via a carbothermal synthesis route. The mixture with $\mathrm{TiO}_{2}$ and carbon resin was reacted at $1500^{\circ} \mathrm{C}$ for 0-45 min under flowing Argon atmosphere. 
The consumption of carbon in carbothermal reactions can be monitored theoretically by measuring the weight loss, which defines the conversion degree. The amount of gasses generated using reaction II is proportional to the carbon having reacted. It is calculated by the ratio of

the measured weight loss to its theoretical maximum value using the following equation:

$$
\propto=\frac{\Delta m}{a m}
$$

Where $(\Delta \mathrm{m})$ is the mass loss measured, (m) the mass of carbon introduced and (a), a coefficient characteristic of the reaction. The value of (a) is determined stoichiometrically [7].

\subsection{Calcium Carbide Investigation}

Calcium carbide is an important compound with a molecular formula $\mathrm{CaC}_{2}$. Pure form of calcium carbide is colorless. It physical appearance depends on the level of purity and the manufacturing technique used. Its properties include a high melting point $\left(2160^{\circ} \mathrm{C}\right)$ and high boiling point $\left(2300^{\circ} \mathrm{C}\right)$. It has a density of $2.22 \mathrm{~g} / \mathrm{cm}^{3}$ at $180^{\circ} \mathrm{C}$. Four phases exist in the calcium carbide (Ca-C) system. Three structurally ordered modifications are known at room temperature and the disordered form IV at high temperature [34]. The crystal structure of $\mathrm{CaC}_{2}$-I is tetragonal while $\mathrm{CaC}_{2}$-II is monoclinic. $\mathrm{CaC}_{2}$-II is considered the low temperature ground state of $\mathrm{CaC}_{2}$ [34]. The common crystalline form I at room temperature is a distorted rock-salt structure.

\subsubsection{Production of Calcium Carbide}

Literature review reveals a number of synthesis routes for making polycrystalline carbides. But the first means and method for the production in an electric arc furnace was discovered and demonstrated through the independent effort of T. L Willson and H. Moissan in 1888 and 1892. 
Although $\mathrm{CaC}_{2}$ was formed as a side reaction product in various industrial processes, it was neither isolated nor recognized. The generally accepted reaction for the formation is represented as reaction (II) below:

$$
3 \mathrm{C}(\mathrm{s})+\mathrm{CaO}(\mathrm{s})<=>\mathrm{CaC}_{2}(\mathrm{~s})+\mathrm{CO}(\mathrm{g})
$$

It was made from lime and coke in the electric furnace at temperature in excess of $2200^{\circ} \mathrm{C}$, using large amount of electric power. Since that time, the basic chemical method used to make the material has not changed and has a heat of reaction of about $445.6 \mathrm{~kJ} / \mathrm{mole}$ at an atmospheric pressure but typically requires $4000 \mathrm{kWh} / \mathrm{t}$ of $\mathrm{CaC}_{2}=923 \mathrm{~kJ} / \mathrm{mole}$ in the prior art (39).

Although calcium carbide has many industrial applications as mentioned before, it is predominantly mass produced for the production of the flammable gas acetylene. It has also recently found new applications as a binder used on mechanical properties of concrete [37].

Unlike all powder ceramics highlighted thus far, most metal carbide synthesis and processing methods reported till date are still completed at high temperature over a longer period of reaction time. An example is seen in the solid state formation of calcium carbide from calcium oxide and carbon precursors found in literature, which involved pressing powder mixture into cylindrical pellets of a $3 / 1$ mole mixture of carbon and lime between $1650^{\circ} \mathrm{C}$ and $1720^{\circ} \mathrm{C}$ at $50 \mathrm{~mm} \mathrm{Hg} \mathrm{CO}$ pressure [7]. The difficulties in this conventional synthesis method are as mentioned before, high temperatures and longer duration of the experiment.

There have been a number of patents granted in a direct effort to develop more efficient techniques and synthesis routes for the production of calcium carbide using different furnace designs and heating mechanisms. One is the preparation of calcium carbide in a shaft furnace by 
W. Van Loon et al. granted in the year 1956. In their work, a mixture of fuel, which the main constituent was carbon such coal, anthracite or charcoal with unslaked lime, dolomite and limestone were fed into the shaft from the top of the furnace with the heat supply by burning part of the fuel present by means of oxygen-containing blast injected into the furnace. The products formed were collected at the lower part of the furnace. This process is complex and not ecofriendly coupled with huge power consumption considering the complexity of their furnace design and the energy sources.

In 1986, Sune Eriksson patented a method of manufacturing calcium carbide from powdered lime and/or limestone. He injected powdered lime material together with a reducing agent, which is optional, with the aid of a carrier gas into a plasma gas generated by a plasma generator. $\mathrm{CO}$ and $\mathrm{H}_{2}$ where used as carrier gas and plasma gas respectively. Coke, charcoal, petroleum coke and/or carbon black are suitable as reducing agent in piece form and the plasma gas used in the process suitably consists of process gas recirculated from the reaction zone. The electric power supplied was $1000 \mathrm{~kW} .4 \mathrm{~kg} \mathrm{CaO} / \mathrm{min}$ was fed in as raw material and as reducing agent $3 \mathrm{~kg}$ coal powder per minute and $0.4 \mathrm{~kg}$ coke per minute. For every $500 \mathrm{~kg}$ technical carbide produced during the experiment, $78 \% \mathrm{CaC}_{2}$ content was realized. The mean electricity consumption was 4 $\mathrm{kWh}$ per kg carbide produced.

El-Naas et al. also reported a 30\% calcium carbide yield due to melting and agglomeration of particles in the plasma zone [3]. In their work, they utilized a semi-batch fluid bed reactor with a DC plasma torch. The precursors used were calcium oxide powder with a mean particle size of about $170 \mu \mathrm{m}$ which was reacted with two types of graphite, coke and methane. Argon gas was used to initiate the plasma and hydrogen gas was then added to increase the power and raise the plasma jet enthalpy. One of the best yield methods reported is by auto-thermal heating process 
through the combustion of chars, with a considerably reduced temperature of about $500^{\circ} \mathrm{C} .97 \%$ $\mathrm{CaC}_{2}$ purity reported. The shortened reaction time and increase thermal efficiency in the conversion of the fine biochar was achieved compared to a $2200^{\circ} \mathrm{C}$ reaction temperature for $1-2$ $\mathrm{h}$ used in a conventional electric arc furnace [36]. These constraints inevitably resulted in high energy cost and therefore make this route less attractive in commercial terms. Another drawback is that the raw material must be in piece form, thus limiting the materials available because these materials are cheaper in fine grained forms.

In addition to high power consumption and production cost usually associated with these conventional techniques: auto-thermal, induction, plasma, electric and rotary arc found in prior arts, impurities such as $\mathrm{Ca}, \mathrm{CaO}, \mathrm{CaCO}_{3}, \mathrm{Ca}(\mathrm{OH})_{2}$, amorphous carbon, graphite, etc are still significantly present in the final product of some the works carried out, with some of these impurities unavoidable considering the use of additive to aid some of these reactions and process. If we consider electrodes in electric furnace that experienced partial worn out, these take part in the process as contributing element in the form of carbon.

Another patented technology developed to address most of the challenges associated with conventional was microwave heating. Prior arts used microwave heating for drying or sintering until Lewis R. Finell and Tom E. Fuller (1985) patented a process for the production of a carbide by-product with microwave energy and aluminum by electrolysis. Their effort at that time was geared towards the principle that materials which are not susceptible to microwave heating will be heated by placing it close enough to another material susceptible to microwave radiation. Their art revealed that when there are two more reactants provided, where one is susceptible to microwave radiation; microwave energy can be employed to bring about a chemical reaction. 
Although there was no mention of the specific energy consumption in the patent abstract, one would expect high energy consumption due to the complexity of their design. Other lacking information in their work included: reaction mechanism, experimental observation, quantification, reducing environment, starting materials form, specific temperature, reaction rate and time.

Fine carbon materials in powder form are very good absorber of microwave (easily heated by microwave radiation), and can heat other materials indirectly, or act as a catalyst and microwave receptor in different heterogeneous reaction $(33,40)$. Chen et al. (1984) reported carbon to heat in $0.2 \mathrm{~min}$ to $1000^{\circ} \mathrm{C}$ temperature. Reagent grades calcium oxide and calcium carbonate are classified as inactive because of their absorbability of microwave irradiation as both reached $200^{\circ} \mathrm{C}$ and $130^{\circ} \mathrm{C}$, respectively, at the rate of $5^{\circ} \mathrm{C} / \min (41)$.

\subsubsection{Kinetics of Calcium Carbide Formation}

Overall reaction representation for the formation of calcium carbide has been given above as reaction II. This reaction falls in the category of non-catalytic gas-solid and solid-solid reactions whereby solid reactants lead to gas and solid products. It is expected that this solid-solid reaction should proceed through gaseous intermediates as in the case of the carbon reduction of metal oxides.

Kemeyama (1956) proposed a two-step reaction mechanism (42):

$$
\begin{aligned}
& \mathrm{CaO}+\mathrm{C} \Leftrightarrow \mathrm{Ca}(\mathrm{g})+\mathrm{CO} \\
& \mathrm{Ca}(\mathrm{g})+2 \mathrm{C} \Rightarrow \mathrm{CaC}_{2}
\end{aligned}
$$


These reactions are considered to proceed in two main steps: initiation and gas-solid reaction. The first step is based on the ionization degree of carbon and subsequent diffusion into the lime particles and forms various calcium vapor sites. Accessible carbon surface area was found to be the main parameter in the stage. Studies show that step three and four reaction rates depend on the area of contact between $\mathrm{CaO}$ and $\mathrm{C}$ (43), which implies that percent yield is proportional to the rate of calcium vaporization. When Inou and Kameyama applied this technique to produce $\mathrm{CaC}_{2}$, percent purity was 97 at $1700^{\circ} \mathrm{C}$ at $100 \mathrm{~mm}-\mathrm{Hg}$ pressure in $1 \mathrm{~h}$ reaction time. Further investigations from past work revealed that in addition to $\mathrm{CO}$ evolution, loss of vaporized calcium also contributed to weight loss. The relationship is approximately linear with time, with the velocity of the reaction doubled for every $32-34^{\circ} \mathrm{C}$ rise in temperature.

The second step of the reaction is diffusion-controlled; vaporized calcium migrates through the layers of formed $\mathrm{CaC}_{2}$. Since this reaction is reversible, the chances of $\mathrm{CaC}_{2}$ decomposition into its constituent's elements increases as temperature increases, with increase and rapid decomposition at $2200^{\circ} \mathrm{C}$.

Tagawa and Sugawara (1962) work on the formation of calcium carbide in a solid state reaction of calcium oxide with carbon in pellet forms, establishing that the reaction followed parabolic kinetics model and that the rate of the reaction was governed by the diffusion of gas through the product layer, as well as the diffusion of solids into the solid bulk and product layers [8].

Brookes, et al (1975) used a zero dimensional model for the formation of calcium carbide in solid pellets based on the argument that the rate of movement of a reaction front through the solid pellet is controlled by the heat transfer across a growing product layer [7]. 
[58] Mu and Hard (1987) examined the process of making acetylene from calcium carbide created using a rotary kiln process, finding that it required less than 30 minutes to achieve lime conversion rates of 50-80\% [6]. Muller (1990) presented a detailed study of the properties and reactions of calcium oxide $(\mathrm{CaO})$ in burnt lime, including the diffusion of carbon into solid lime and presented composite reactions of $\mathrm{CaO}$ and $\mathrm{C}$ in solid and liquid states $[29,58]$. Most recently, $\mathrm{Li}$ et al., (2012-13) explored the reaction mechanisms of $\mathrm{CaC}_{2}$ production from pulverized coke and $\mathrm{CaO}$ at low temperatures, finding that there appeared to be either 2 or 3 distinct reaction mechanisms taking place, depending upon the initial ratio of carbon to $\mathrm{CaO}$. They also studied the influence of the major minerals present in coal-derived coke upon the reaction of $\mathrm{CaO}$ with coke. In this second study, they found that, in general, the presence of minerals in coal-derived coke reduced the formation of the desired $\mathrm{CaC}_{2}[52,53,58]$. In general, the previously-mentioned authors agree that the formation of calcium carbide from carbon and lime is governed by the reactions II, III, IV and V as described in this write-up.

It is also widely accepted that once formed, $\mathrm{CaC}_{2}$ can either dissociate into calcium and carbon via reaction (IV), or react with $\mathrm{CaO}$ per reaction (V).

$$
\mathrm{CaC}_{2}+2 \mathrm{CaO} \rightarrow 3 \mathrm{Ca}+2 \mathrm{CO}
$$

Inability by some researchers to account for massive circulation of vaporized calcium as often been pointed out in literature, usually brings conflict between research and production outcomes as we have seen in all examples mentioned thus far.

Most, if not all, of the previous authors have studied the reactions listed in an effort to determine the reaction rates of the chemical kinetics involved in the formation of $\mathrm{CaC}_{2}$. In these cases, 
these previous authors have attempted to fit traditional reaction rate models to experimental data with varying levels of success. However, most of these rate models are limited to a single global reaction, and it is becoming widely accepted that the formation of $\mathrm{CaC}_{2}$ involves a multi-step reaction mechanism [58].

Part of the work completed in collaboration with this work is to present two new methods for modeling the chemical reaction mechanisms associated with the formation of Calcium Carbide from Coke and Lime. The first of these new models is referred to as the progress variable model. This model utilizes equilibrium mass fraction data to determine the direction that the reaction must proceed in order to reach a state of chemical equilibrium [58]. The second model, known as the multi-step reaction model, is based upon modeling the kinetic rates of the calcium carbide formation and disassociation reactions [58].

In the current study, a general modeling paradigm is retained such that once validated it can be implemented into readily available multi-dimensional computer codes to simulate $\mathrm{CaC}_{2}$ formation in complex reactors [58].

The constituents starting materials, synthesis technique used as well as the reaction temperature also influence the activation energy of the reactions. To obtain pure $\mathrm{CaC}_{2}$ free of $\mathrm{CaO}$ impurities, elemental calcium with graphite at $1070^{\circ} \mathrm{K}$ can be reacted in inert environment using a conventional electric resistance furnace for 16hrs (44).

Ahlers and Ruschewit repeated the same experiment mentioned above in a modified domestic microwave oven using a tablet pressed from $0.04 \mathrm{~g} \mathrm{Ca}(1 \mathrm{mmol})$ and $0.036 \mathrm{~g}$ graphite $(3 \mathrm{mmol})$ (Mfg.: Alfa, 99.6\%, 100-200 mesh; Ca: Aldrich, 99.99\%) stirred for 15 minutes at maximum 
microwave power $(800 \mathrm{~W})(45) . \mathrm{CaC}_{2}(90 \%$ by weight), $\mathrm{CaO}(5 \%)$ and unreacted graphite were obtained [45].

Although the studies mentioned above may give sufficient explanation of the kinetics of $\mathrm{CaC}_{2}$ formation, there is still need to accurately account for experimental conditions such as solid-solid surface contact, pellet size and compaction and morphological and microstructural differences. The reaction taking place using microwave energy may not follow the reaction paths as in conventional methods, as this fundamentally rely on the dielectric properties of the compounds present to drive the reaction and where this is not initially possible, a susceptor is used. It may be possible that the reactions proceed through different routes to obtain the final product due to the rapid reaction times, lower temperatures and localized heating, without an intermediate step.

It is the primary aim of the present study to investigate the feasibility of the solid phase formation of calcium carbide, characterize the reaction rates of carbon and calcium oxide precursors with various size ratios and chemistries, develop a method to obtain kinetic reaction constants and draw a correlation between the particle characteristics and the kinetic constants. This investigation includes obtaining reaction kinetics data to assist in defining the reaction mechanism and difference in kinetic constants of the reaction through the use of microwave heating compared to conventional convection heating. 


\subsection{EXPERIMENTAL DETAILS}

\subsection{General Experimental Description}

The overall details of the experimental technique and procedure are described. Microwave and conventional furnaces were used to prepare calcium carbide in a direct reaction of graphite; coal and coal char as the carbon source with calcium oxide (lime) as the calcium source. These experiments were carried out at different reaction temperatures, rates and hold times, weight (molar) fraction and reaction location (within the furnace). For experimental works carried out in the microwave, after mounting and aligning the sample for top surface temperature measurement, the chamber was evacuated and backfilled with argon gas three times prior to any run and a argon positive pressure was maintained throughout the run.

The products obtained were characterized using the X-ray Photoelectron Spectrometer (XPS) and X-Ray Diffraction (XRD). The phase content from the XRD patterns was qualitatively analyzed using Rietveld technique. The phase content was also characterized using a water emersion process, where the gas produced through hydrolysis of final product from the furnace reaction in distilled water was collected and analyzed using a Gas Chromatograph (GC). The Scanning Electron Microscope (SEM) and Energy dispersive X-Ray Diffractometry (EDX) were also used.

\subsection{Raw Materials and their Characterization}

The starting materials (powders) were characterized using X-Ray Diffraction (XRD), Scanning Electron Microscope (SEM), Thermogravimetric Analysis (TGA) and Malvern Mastersizer 2000 particle size analyzer. The results of their particle size distribution measurements are recorded in 
Table 3.1. Media to charge ratio used is 10:1. To obtain significant decrease in powder particle size distribution, the media to charge ratio needs to be doubled not the duration.

Table 3.1 Characteristics of experimental starting materials.

\begin{tabular}{|c|c|c|c|c|c|}
\hline \multirow{2}{*}{$\begin{array}{l}\text { Powder mix: } \\
(\mathrm{CC} 2 \mathrm{M}) \\
\text { Ball milling duration: }\end{array}$} & \multicolumn{3}{|c|}{ CaO-Graphite (CGM) } & \multirow{2}{*}{$\begin{array}{l}\mathrm{CaO}-\mathrm{Coal}(\mathrm{CCM}) \\
\begin{array}{c}24 \\
\text { (hr.) }\end{array}\end{array}$} & \multirow{2}{*}{$\begin{array}{l}\mathrm{CaO}-\mathrm{CoalChar} \\
24 \\
\text { (hr.) }\end{array}$} \\
\hline & 24 & $\begin{array}{c}96 \\
\text { (hr.) }\end{array}$ & 504 & & \\
\hline \multicolumn{6}{|l|}{$\begin{array}{l}\text { Particle Size } \\
\text { Distribution, PSD }\end{array}$} \\
\hline $\begin{array}{ll}(\mu \mathrm{m}) & \mathrm{D}_{10} \\
& \mathrm{D}_{50} \\
& \mathrm{D}_{90}\end{array}$ & $\begin{array}{l}3.39 \\
13.72 \\
37.36\end{array}$ & $\begin{array}{l}1.72 \\
11.77 \\
32.86\end{array}$ & $\begin{array}{l}1.83 \\
8.50 \\
23.1\end{array}$ & $\begin{array}{l}1.25 \\
2.75 \\
7.05\end{array}$ & $\begin{array}{c}2.26 \\
10.56 \\
59.27\end{array}$ \\
\hline $\begin{array}{l}\text { Theoretical Density } \\
\qquad\left(\mathrm{g} / \mathrm{cm}^{3}\right)\end{array}$ & & 2.41 & & 1.84 & 1.59 \\
\hline
\end{tabular}

Reagent grade calcium oxide and graphite powders used were all purchased from Alfa Aesar, (Johnson Matthey Company). The lignite coal used in this work was obtained from WVU Coal Research Corporation and it contained about 5\% ash. The SEM image of this coal is presented in Figure 3.1 .

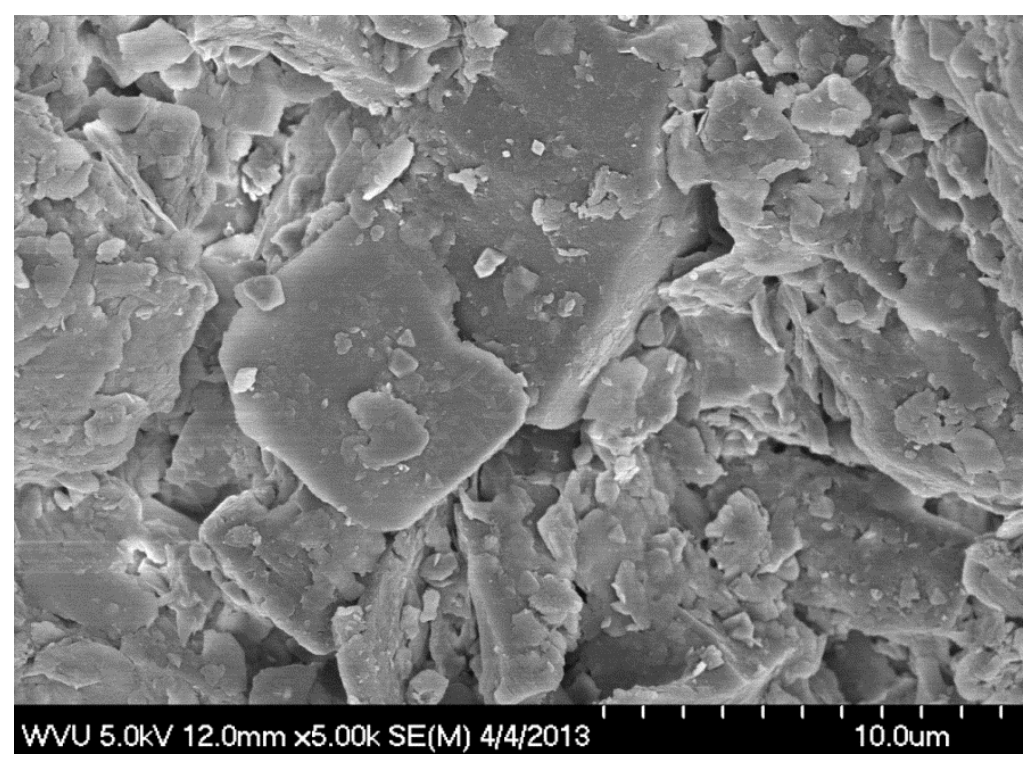

Figure 3.1 SEM of carbon coal obtained from WVU Coal Research Corporation. 
The essence of this is to distinguish the surface area of both coal and graphite carbons as the accessible surface area influence the reaction rate.

An SEM image, XRD patterns and XPS survey and detailed scans of the $3 / 1$ stoichiometry mixture of the starting powders according to the generally accepted reaction are shown in Figure 3.2, 3.3 and 3.4 respectively. The mixture of these powder particles of the constituents' materials are well mixed, with composition identified and the expected phases defined as well. XRD Rietveld refinement quantification technique used revealed the mixture consisted of $62.50 \pm 1.5$ mass $\%$ of lime and $37.50 \pm 1.5$ mass $\%$ of graphite.

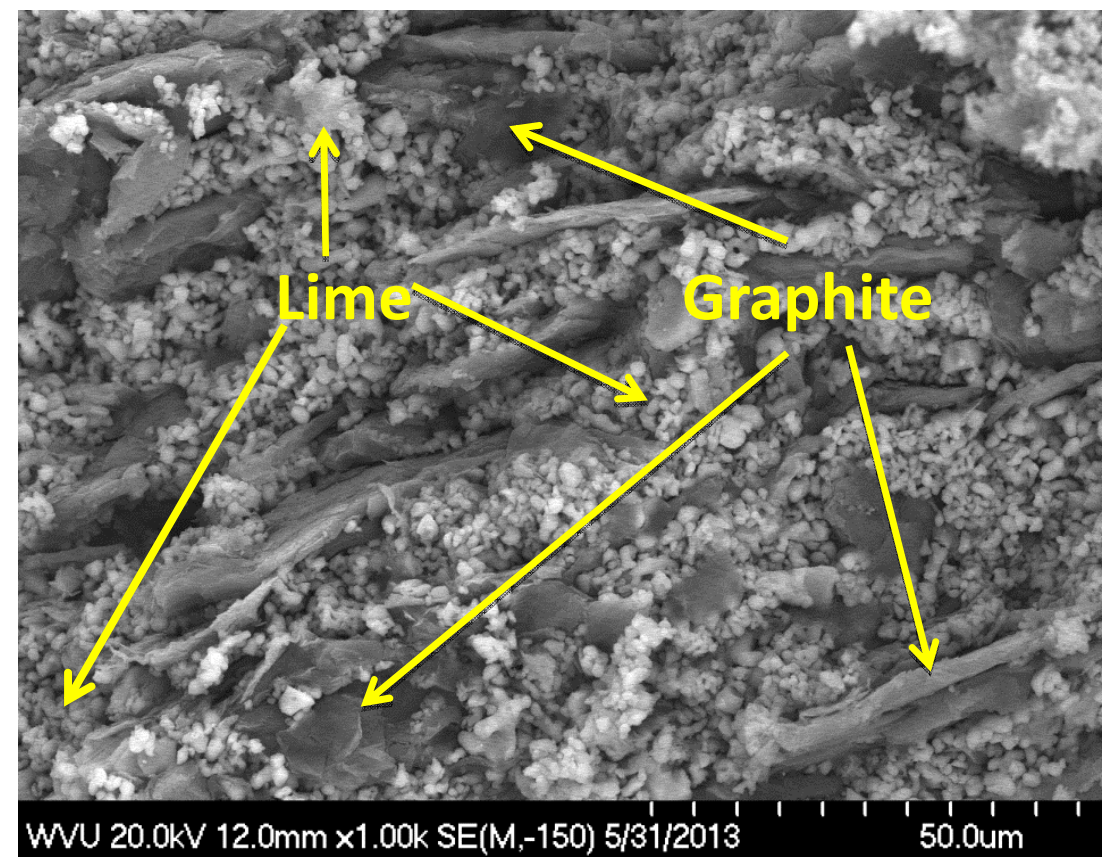

Figure 3.2 SEM back-scattered image of 3:1 stoichiometric mixture of graphite and lime powder (Alfa Aesar). 


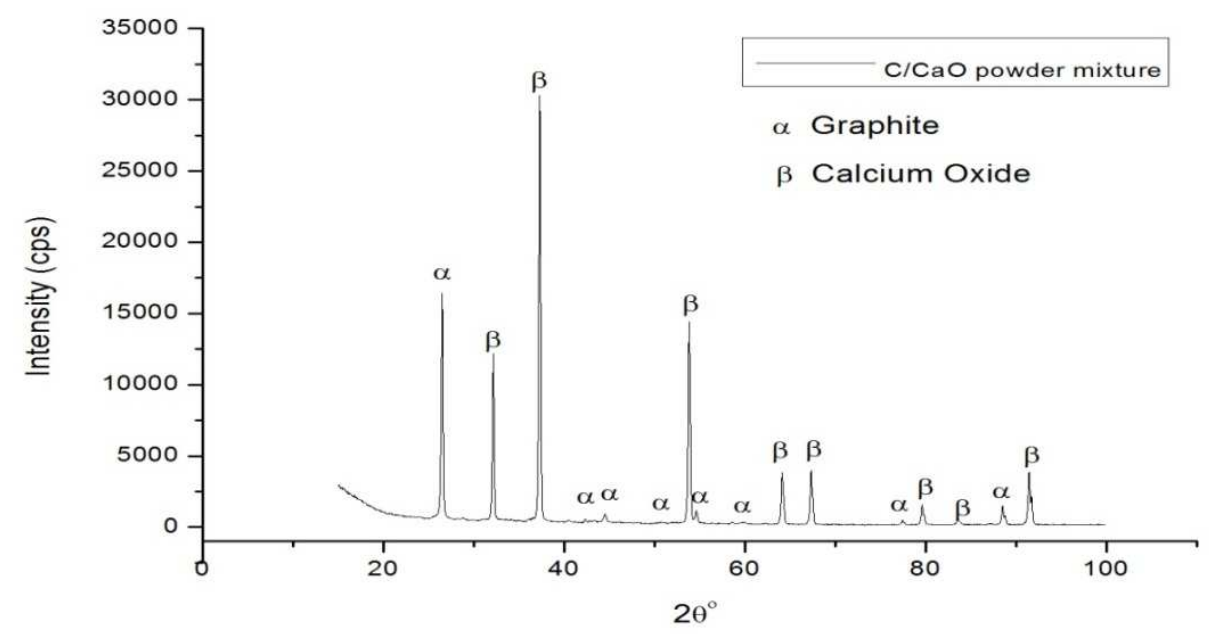

Figure 3.3 XRD patterns of reagent grade calcium oxide (lime), graphite and the 3:1 stoichiometric mixture of both graphite and lime powder (Alfa Aesar).

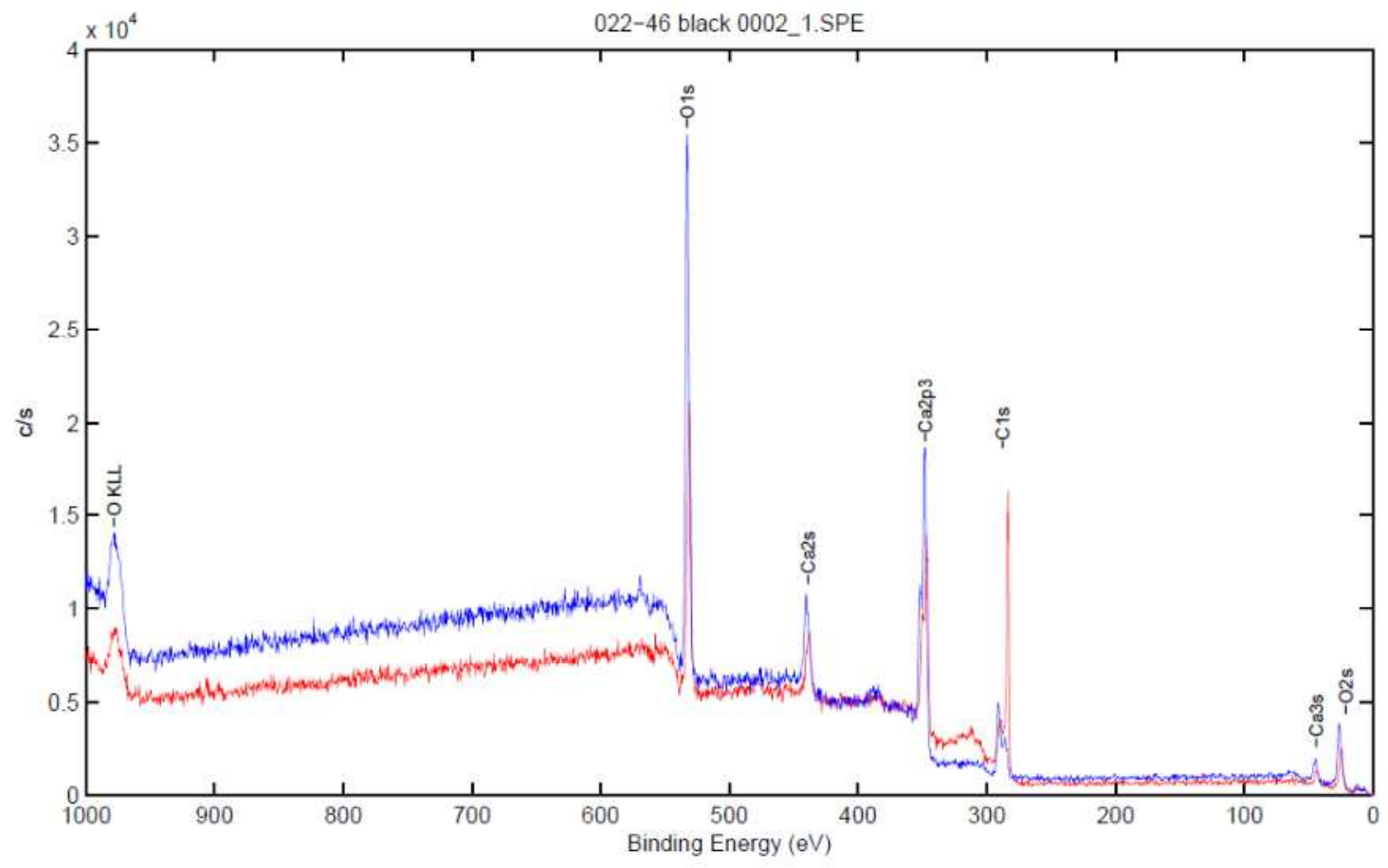

Figure 3.4: XPS survey scan for 3:1 stoichiometric mixture of both graphite and lime powder (Alfa Aesar supplies); Stable carbon peak formed at $284.5 \mathrm{eV}$ as revealed from the detailed scans for $\mathrm{C} 1 \mathrm{~s}, \mathrm{Ca} 2 \mathrm{p}$ and $\mathrm{O} 1 \mathrm{~s}$. 


\subsection{Powder Processing}

As-received reagent grade calcium oxide (average particle size of $4.62 \mu \mathrm{m}$ ) and graphite (average particle size of $19.28 \mu \mathrm{m}$ ) powders were ball-milled for 24 hours, 96 hours and 504 hours in a suspension of isopropanol (1-Propanol), contained in a plastic bottle with zirconia media charge to make three batches with different particle sizes. The charge to mass ratio used was 10:1. The milling of the powder for furnace reactions was performed with the parameters summarized in the Table 3.2 below. After milling, the suspension was dried using a "Rotavap" evaporator (in vacuum at about $60^{\circ} \mathrm{C}$ ). The powder was left inside a vacuum oven at $90^{\circ} \mathrm{C}$ for 2 hours to ensure that it was completely dry. The dried powder was sieved using a $250 \mu \mathrm{m}$ sieve and only the material which passed through the sieve was used for further processing.

Table 3.2: Summary of milling parameters for 3:1 stoichiometric mixture of starting materials.

\begin{tabular}{|l|l|}
\hline Milling apparatus & Horizontal rotary ball mills \\
\hline Milling speed & $180 \mathrm{rpm}$ \\
\hline Milling time & $24,96,504$ hours (per batch) \\
\hline Total mass of powder per batch & Total mass of powder 50g \\
\hline
\end{tabular}

To improve surface contacts of the mixture to be reacted, cylindrical pellet discs of $12.77 \mathrm{~mm}$ in diameter and varied thickness (approximately $1 \mathrm{~mm}-2.5 \mathrm{~mm}$ ) were made by using an 11-ton uniaxial press (1000 psi- $7000 \mathrm{psi})$. Approximately, 0.2-0.6 grams of powder was pressed in the $10 \mathrm{~mm}$ hardened tool steel die/punch set to obtain various green samples used for this work. The calculations for the theoretical density (see equation 3.1) were carried out based on the rule of mixtures formula: 


$$
\rho_{\text {theoretical }}=\frac{\sum m i}{\sum \frac{m i}{\rho i}} \times 100
$$

The densities ( $\rho$ ) used for this calculation were $2.09-2.23$ and $3.35 \mathrm{~g} / \mathrm{cm}^{3}$ for graphite and lime, respectively. The fractional mass is represented as $\mathrm{m}_{\mathrm{i}}$, while $\rho_{\text {theoretical }}$ represented the theoretical density. The prepared samples were placed inside a crucible, with and without a lid, and the sample was introduced into the furnace and positioned in the hot zone. After each run, the reacted samples are pulled out of the furnace at a temperature below $300^{\circ} \mathrm{C}$ but above $100^{\circ} \mathrm{C}$ to prevent interaction with air moisture and placed in a transfer vacuum container. Weight loss and densification values are recorded before the samples are reacted and vacuum sealed in a plastic bag (and stored in a $0 \%$ humidity glove box). Further processing was carried out within the glove box.

The dimensions and weight of samples were averaged to measure the green density of the pellets at $1.70 \mathrm{~g} / \mathrm{cc} \pm 0.02$ at a $95 \%$ confidence level. Isothermal reaction kinetics were studied from $1000^{\circ} \mathrm{C}$ to $1600^{\circ} \mathrm{C}$ for microwave heating and $1500^{\circ} \mathrm{C}$ to $1800^{\circ} \mathrm{C}$ for conventional heating.

\subsection{Reaction Apparatus Used for Study}

\subsubsection{Reaction Crucibles}

Round alumina (20 ml x $15 \mathrm{ml})$, square graphite $(70 \mathrm{ml} \times 30 \mathrm{ml}$ x $15 \mathrm{ml})$ and zirconia combustible boat ( $100 \mathrm{ml} \times 20 \mathrm{ml} \times 15 \mathrm{ml})$ crucibles were used. The boat and square crucibles can contain four pellet samples in one batch run. 


\subsubsection{Reaction Furnace}

Both conventional and microwave furnaces were used for this study. Figure 3.5 shows a picture of the Nabertherm high-temperature tube furnace with low-voltage transformer and thermistor (model series RHTH with a C799 standard vacuum tube of $40 \mathrm{~cm}$ inside diameter, $50 \mathrm{~cm}$ outer diameter and $99 \mathrm{~cm}$ long) used in this work. The $\mathrm{MoSi}_{2}$ heating elements are in an easy to replace hanging arrangement; can attain furnace chamber temperatures of maximum $1800{ }^{\circ} \mathrm{C}$ $\left(3272{ }^{\circ} \mathrm{F}\right)$. Its insulation materials are made of vacuum-molded fiberboards, allowing for energy-saving operation and fast warm-up times because of low heat storage and thermal conductivity. Experimental work can be done in a protective gas atmosphere, vacuum or even in combustible gases. Temperature measurement is read by thermocouple type B and the switchgear with controller separate from the furnace with a detach floor cabinet. The temperature profile of a typical run at $1800^{\circ} \mathrm{C}$ and $60 \mathrm{~min}$ hold time is shown in figure 3.6.

Additionally, an MTI GSL-1500X-50 CE certified compact high temperature 9.84" O.D tube furnace using $99.8 \%$ high purity processing alumina tube and $\mathrm{SiC}$ heating elements was also used as back up furnace to complete reactions only at $1500^{\circ} \mathrm{C}$. It has a vacuum sealing assembly which connects with 1/4" gas hose to supply gas. The temperature of the MTI GSL1500X tubing furnace is controlled by high precision SCR (Silicon Controlled Rectifier) digital controller with accuracy $+/-1^{\circ} \mathrm{C}$ and 30 segments programmable up to $1500^{\circ} \mathrm{C}$. Both furnaces heating and cooling rates were within the range of $3-5^{\circ} \mathrm{C} /$ minute. 


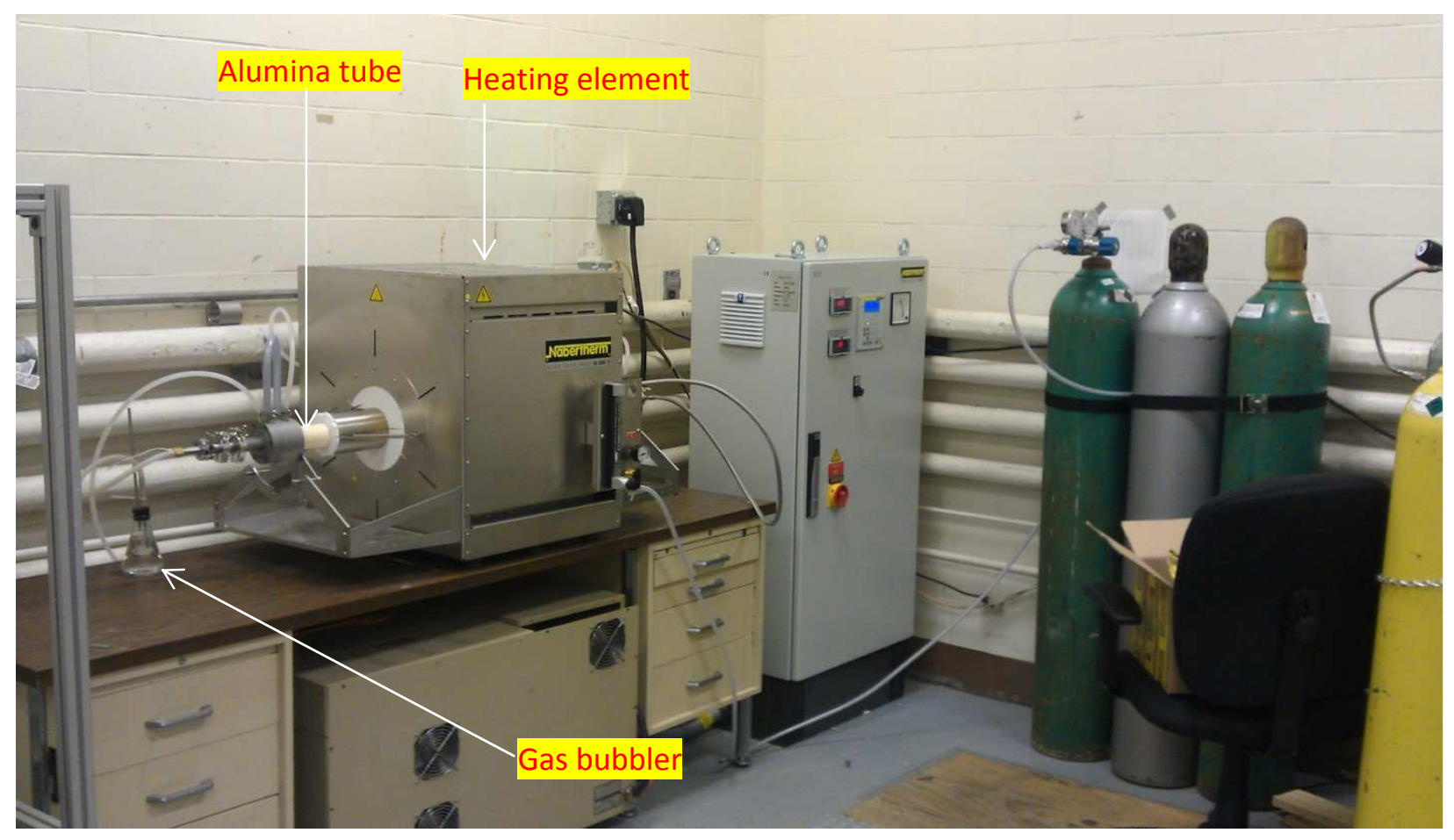

Figure 3.5: Low Voltage Nabertherm High-Temperature tube furnace model series RHTH.

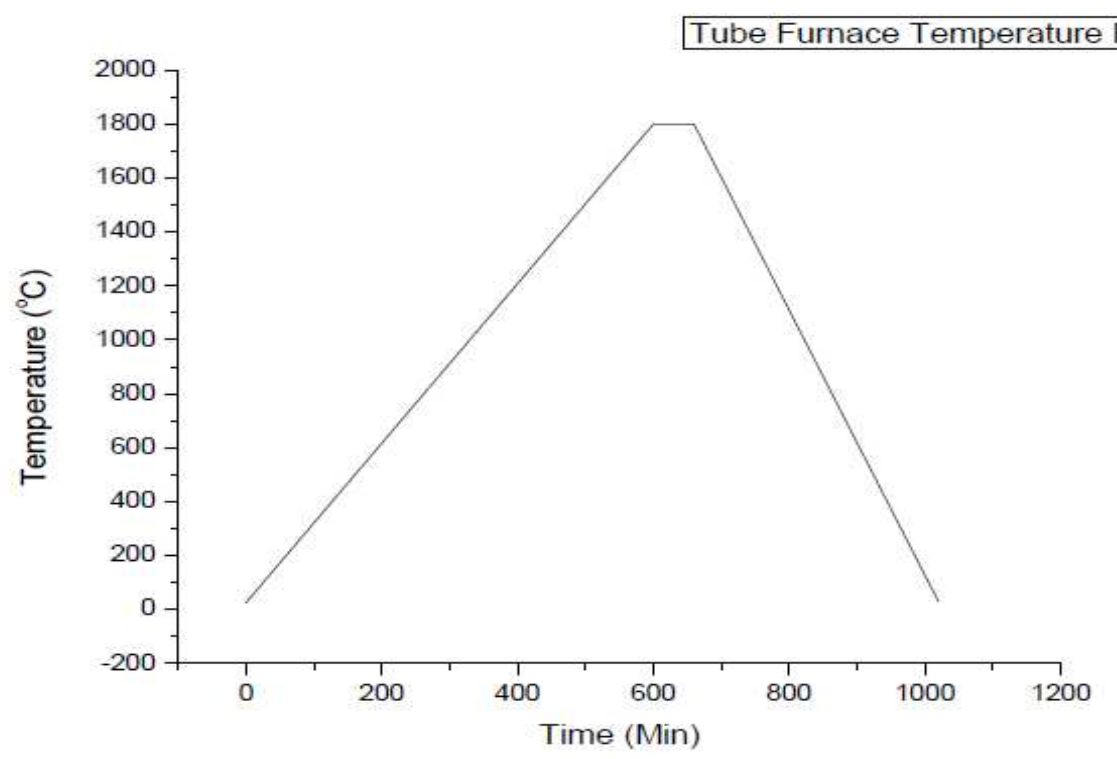

Figure 3.6: Typical temperature profile of the conventional furnace; this represent a temperature for experiment ramped up to $1800^{\circ} \mathrm{C}$ with 60 minutes exposure time then allowed to cool down to room temperature. 
Figure 3.7 and 3.8 show the microwave furnace system used for this study and a heating profile respectively. Figure 3.9 shows the design of the insulating casket for microwave sintering. It is very vital to select the right ceramic package to prevent loss of heat, arcing, unwanted reactions etc. The microwave furnace used for this study is an industrial multi-mode microwave system that has the capacity to run at $12 \mathrm{~kW}, 2.45 \mathrm{GHz}$ with two adjacent microwave windows, three microwave generation units, a turn table and vacuum and gas system. The chamber volume is about 441.150 liters. The input power was adjusted manually to control the sintering temperature and heating rate. The samples were placed in a high alumina crucible surrounded by a cylindrically-shaped insulating casket made from alumina silicates refractory materials. A small hole of $1.3 \mathrm{~cm}$ in diameter on the top cover of the casket was made for non-contact temperature measurement. The operating reaction temperatures in the microwave furnace were measured with a digital infrared pyrometer. The pyrometer requires the user to manually set an emissivity value for the sample being measured as well as manual focusing on the sample through the hole on the lid of the insulation package. It was required to calibrate the pyrometer using a conventional furnace to ensure accurate temperature measurements. 


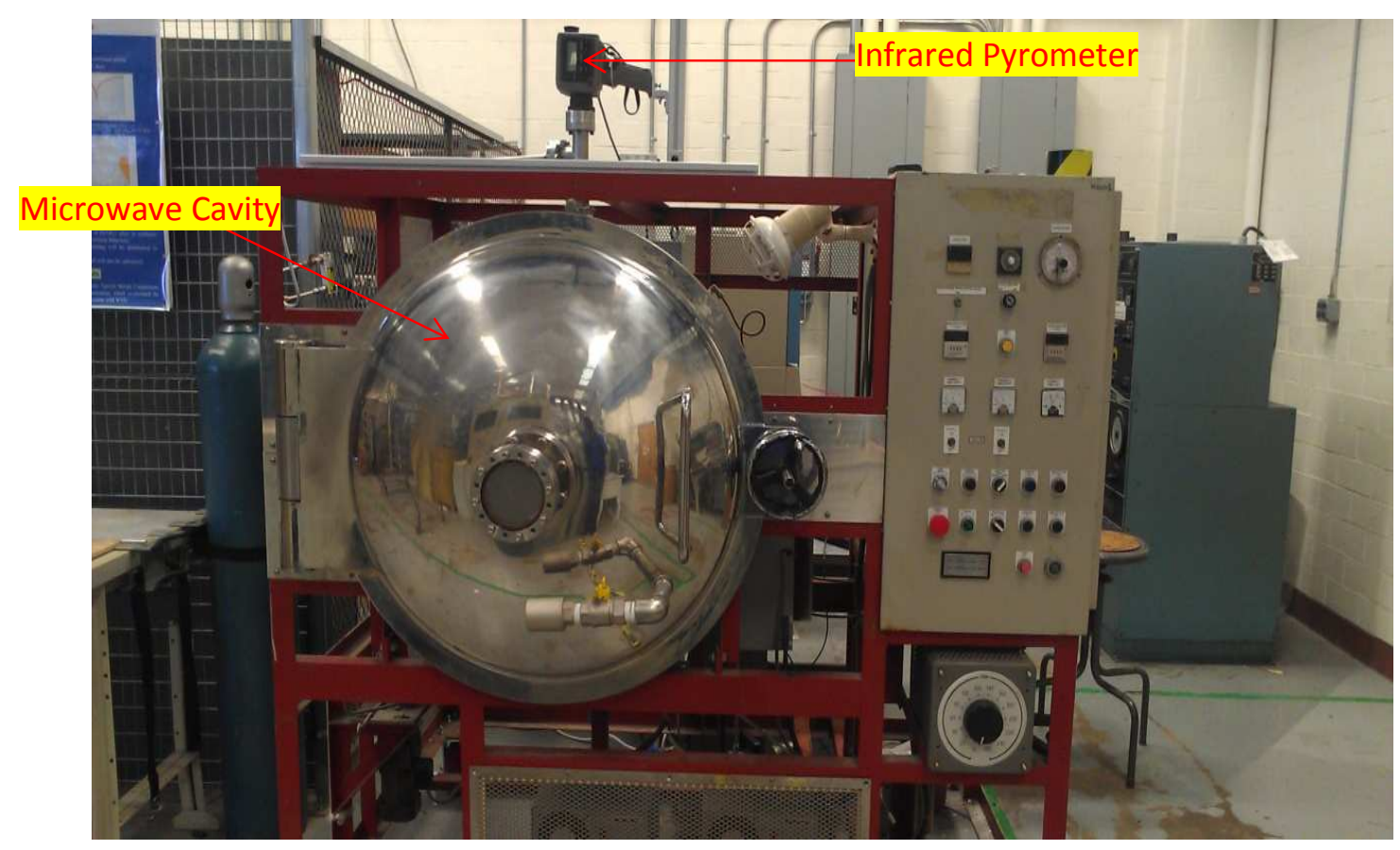

Figure 3.7: Microwave Furnace Heating System; 12kW, 2.45GHz donated by Hadron Technologies Inc.

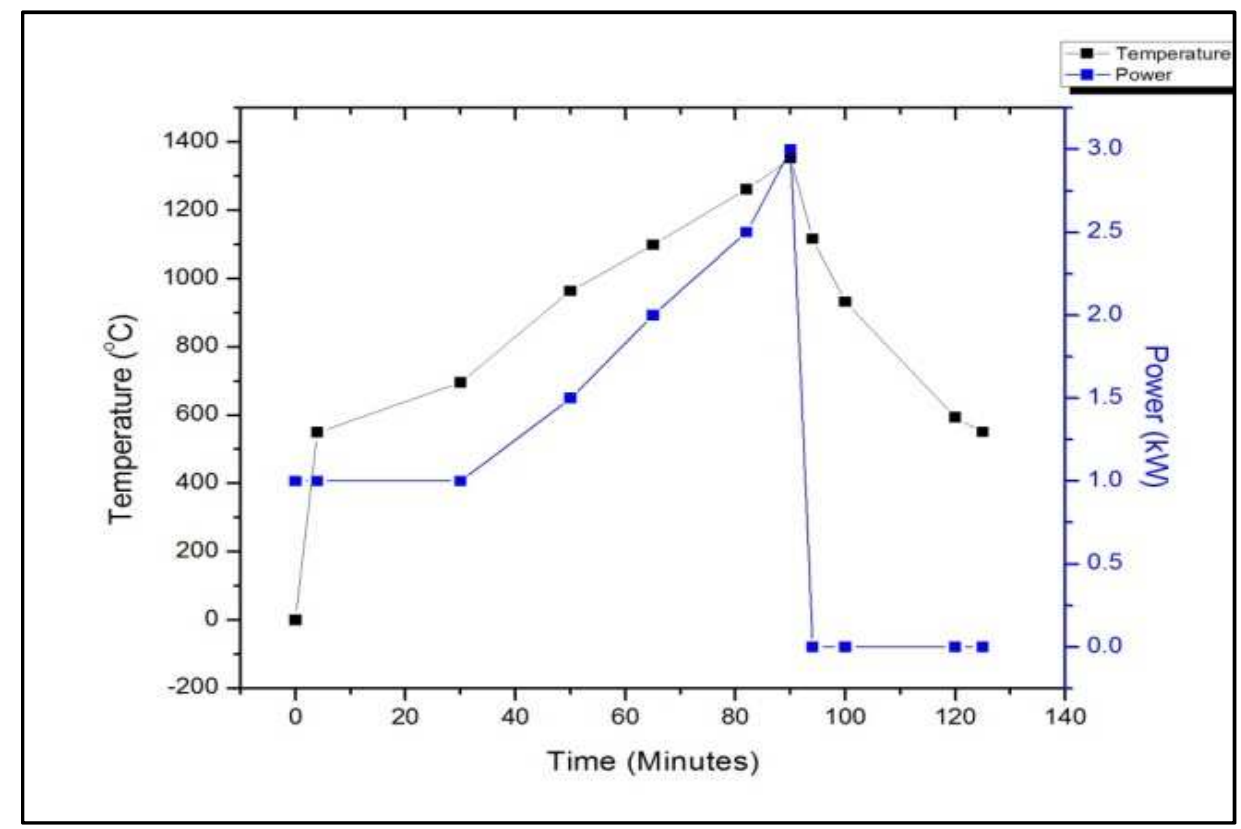

Figure 3.8: Typical temperature profile obtained during microwave heating of $\mathrm{C}-\mathrm{CaO}$ mixture and power level used. 


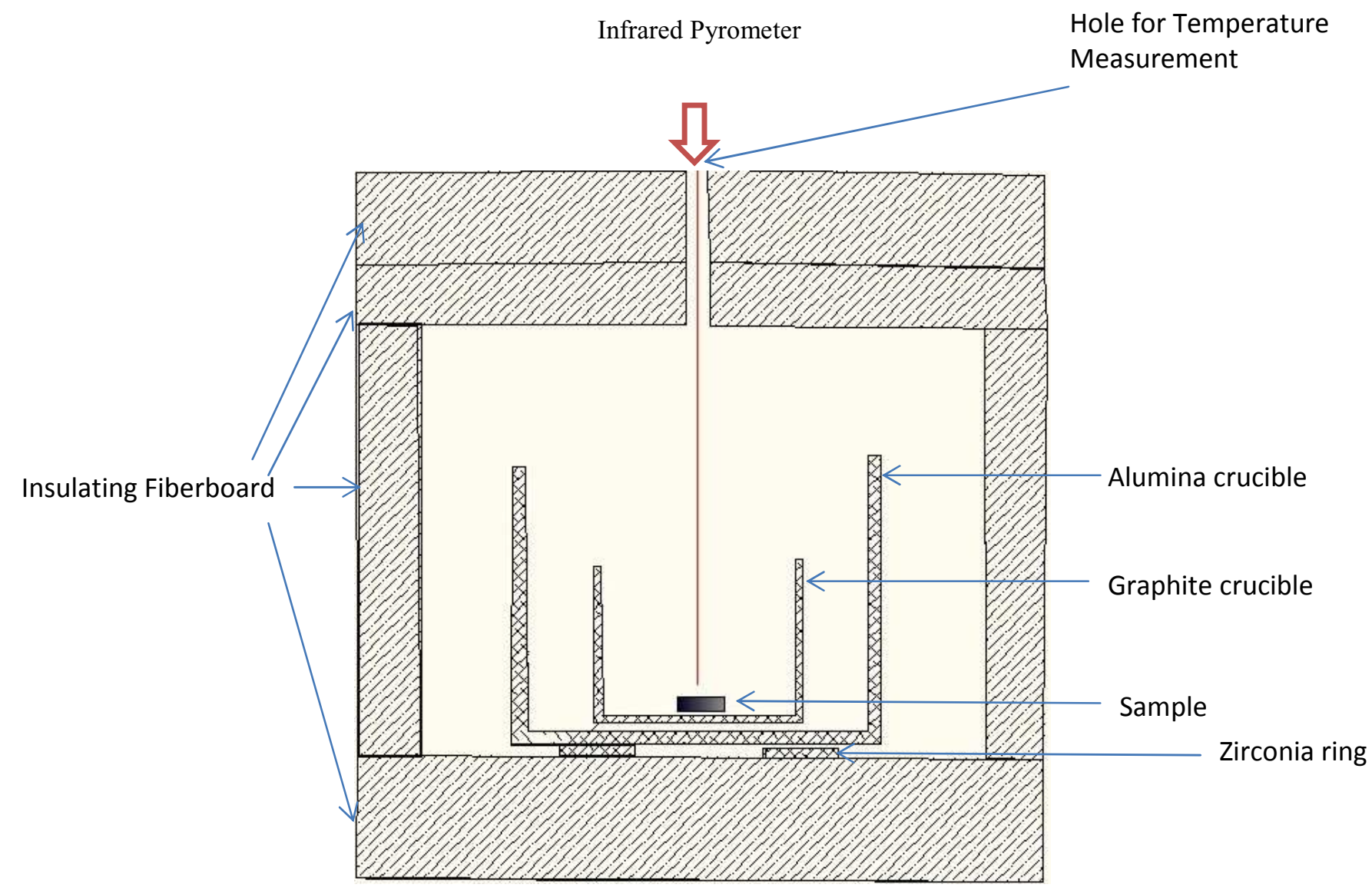

Figure 3.9: Insulating package for microwave reaction (Note: Not drawn to scale).

\subsection{Experimental Procedure}

Heating was conducted in both conventional furnace (electric resistance furnace with $\mathrm{MoSi}_{2}$ heating elements) and multimode microwave furnace in argon environment at temperatures ranging from $1000^{\circ} \mathrm{C}$ to $1800^{\circ} \mathrm{C}$. The heating and cooling rates for conventional sintering were maintained at $3^{\circ} \mathrm{C} / \mathrm{min}$. The heating rate for the microwave furnace is unknown below $550^{\circ} \mathrm{C}$ due to the limitations of the infrared pyrometer, but approximately varied between 45 and $1{ }^{\circ} \mathrm{C} / \mathrm{min}$ above $550{ }^{\circ} \mathrm{C}$. The hot zone was determined to be $47.5 \mathrm{~cm}$ from either end of the alumina tube in the tube furnace. Various types of reaction crucibles were used in this study as mentioned before, 
to contain the samples. Initial experiments completed in the crucibles without lids inside the tube furnace led to degradation and subsequent loss of tubes as a result of calcium vaporization coupled with repeated heating. The reduction of the effective volume and use of lid has however eliminated this challenge. The small closed graphite pots minimized diffusion through them.

In the case of microwave runs, melting of insulating package and reaction crucibles occurred due to random hot-spots inside the microwave cavity. To curb this unwanted situation, a 1 inch offset of zirconia ring pedestal and graphite-alumina ceramic system combination was incorporated into the insulating package design.

For a regular run in the microwave, the furnace cavity was evacuated to 1 bar pressure level and backfilled with a research grade argon gas three times and then the flow rate of the gas into the microwave furnace throughout each run was maintained at $2 \mathrm{~L} / \mathrm{min}$. A bubbler was installed below the furnace chamber for leak testing and ensuring positive pressure is maintained. Upon conclusion of each test, the resulting samples were removed and stored within a vacuum container. The samples were retrieved from the container to measure density and densification and final weight (for weight loss information) and transferred to the glove box for further storage before microscopy and phase characterization.

\subsection{Analysis of Samples}

\subsubsection{Weight Loss Measurement}

Weight loss characterization involved recording the mass of sample in both pellet and powder forms before introducing it into the furnace for every run and also after taking it out of the furnace. The same information is retrieved by weighing the container vessel, including ceramic setter and graphite foil, before and after every run to determine the percent weight loss. 


\subsubsection{Density Measurement}

The density of all of the reacted materials was measured using the Archimedes method and also taking thickness and width dimensional information to determine Geometrical Density. The first step in Archimedes was to measure the dry mass of the sample. The sample was then dropped in a plastic cup half-filled with 2-Isopropanol, placed under vacuum at room temperature for 15 minutes. Then the mass of the samples suspended in 2-Isopropanol was measured. The sample was then taken out of the 2-Isopropanol filled cup and excess 2-Isopropanol was removed from the sample by dabbing it with a cloth. The mass of this "2-Isopropanol-impregnated" sample was then measured. The calculations for this method are briefly outlined in equations (3.2) and

$$
\begin{aligned}
& \text { Bulk density }(\mathrm{g} / \mathrm{cm} 3): \rho=\frac{(d)\left(\rho_{\text {ipa }}\right)}{\left(w_{e}-w\right)} \\
& \text { Open porosity (\%): } P_{o}=\left[\frac{w_{e}-d}{\left(w_{e}-w\right)}\right] 100 \%
\end{aligned}
$$

Where,

$\rho_{\text {IPA }}(\rho$ Isopropan-2-ol $)=$ density of Isopropan-2-ol at room temperature $=0.786 \mathrm{~g} / \mathrm{cm} 3$, $\mathrm{d}=$ dry mass of the sample, $\mathrm{w}=$ mass of IPA-impregnated sample in IPA, $\mathrm{w}_{\mathrm{e}}=$ mass of IPA-impregnated sample. 


\subsubsection{X-ray Diffraction Phase Analysis: Qualitative and Quantitative}

Samples in the form of powder, solid and crushed pellet, before and after reaction, were scanned in a PANalytical XRD using $\mathrm{Cu}-\mathrm{K} \alpha$ radiation. For the Rietveld calculations, it was necessary to scan over a wide range from $15^{\circ}$ through $110^{\circ}$ for all measurements using a step-size of $0.05^{\circ}$, and holding for 25 seconds per step. The quantitative phase analysis was made with the X'pert Highscore Plus software using the structural data given in the ICDD database.

The weighted profile $\mathrm{R}$-value, $\mathrm{R}_{\mathrm{wp}}$ can be defined as the mean weighted difference between the calculated diffraction pattern (fitted by a least squares approximation up to a best fit) and the measured XRD pattern. This parameter is calculated as shown in equation 3.4.

$$
R_{w p}=\left[\frac{\sum w_{i}\left(y_{i o}-y_{i c}\right)^{2}}{\sum w_{i} y_{i o}^{2}}\right]^{1 / 2}
$$

Where,

$\mathrm{W}_{\mathrm{i}}=$ mass of component phase $\mathrm{I}$,

$\mathrm{y}_{\mathrm{io}}=$ intensity of experimentally observed XRD peaks for phase I,

$\mathrm{y}_{\mathrm{ic}}=$ intensity of Rietveld calculated XRD peak for phase $\mathrm{i}$.

Thus, $R_{w p}$ is representative of the error in the result of the Rietveld calculation. An $R_{w p}$ value of less than or equal to $10 \%$ was considered an acceptable agreement between experimental spectra and calculated spectra. However, it is recommended that the visual examination of calculated profile and experimental profile match be done. Results were accepted even if the $R_{w p}$ value was still more than $10 \%$ provided that all peaks identified are accounted for and the $\mathrm{R}_{\mathrm{Bragg}}$ value, which does modify the R for a specific phase, which should be less than $<50$. 


\subsubsection{Scanning Electron Microscopy (SEM)}

Scanning electron microscopy (SEM) was used to analyze the microstructural development, grain size and the different phases presence in the sample mix before and after reactions. In this work, reacted samples are usually broken into two pieces before sputtering deposition of a thin layer of gold or platinum to increase sample conductivity and also protect the integrity of the surface.

\subsubsection{Gas Analytical Technique}

Hydrolysis testing was performed to quantify yield percent of calcium carbide synthesized. In this test, acetylene or/and hydrogen is generated by placing the reactant on a hanging pan inside a $125 \mathrm{~cm}^{3}$ flask containing $100 \mathrm{~cm}^{3}$ of water, attached to a pressurized $500 \mathrm{~cm}^{3}$ capacity steel cylinder with a vacuum gauge and valves at both ends (as shown in figure 3.10). When the sample is dropped, the valve end attached to the flask is opened to collect the generated gas. Pressure and temperature before, during and after the reaction were recorded, and the collected gas sample was analyzed with a gas chromatograph (GC) to determine the gas composition.

The GC technique adopted can detect gaseous impurities and also quantitatively determine the mixture constituents. For GC experiments, gas samples were taken by attaching an air-tight syringes (Model: 1100 Gastight syringes, Hamilton Company, Reno, NV) to the gas sampling port. Three gas samples were taken at 20 minute intervals after each run and then tested in a gas chromatograph (Model: Clarus GC 580, Perk Elmer Waltham, MA), shown in figure 3.11, to determine the gas composition. A gas standard (Catalog: 501662 Gas standard, Supelco, PA, USA) was used for calibration of the gas chromatograph for detecting carbon monoxide, carbon dioxide, methane, ethane, ethylene $\&$ acetylene each at $1 \%$ in nitrogen. Another gas standard 
(Product: N6107201 Gas standard, Perkin Elmer, Shelton CT, USA) was used to calibrate the gas chromatograph for hydrogen.

Argon was used as the carrier gas for this system since the value of the thermal conductivity of helium gas is closed to that of hydrogen gas. 


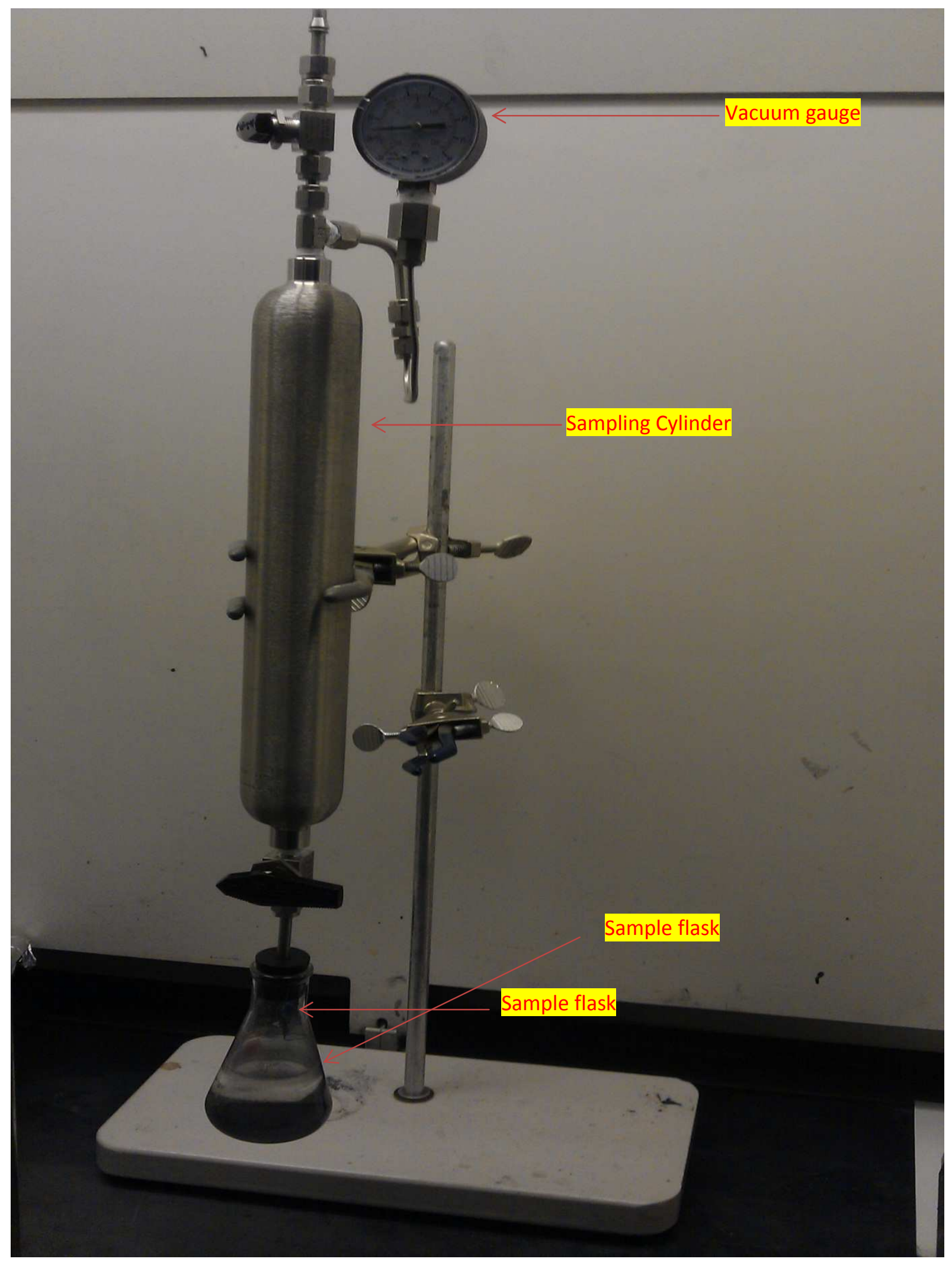

Figure 3.10: Gas generation and collection apparatus (Vacuum pump is detached). 


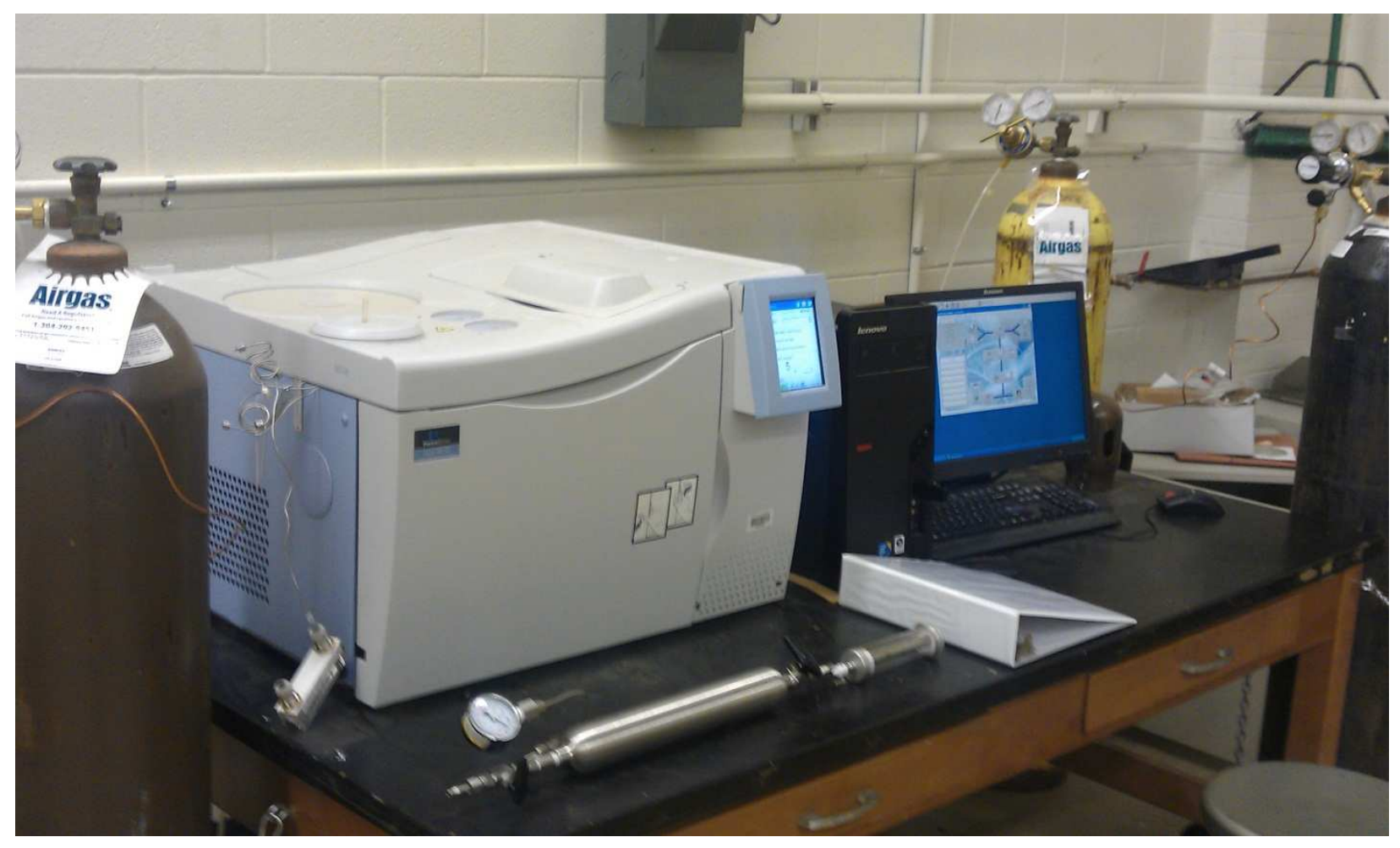

Figure 3.11: PerkinElmer ${ }^{\circledR}$ Clarus ${ }^{\circledR} 580$ Gas Chromatograph (GC) used to analyzed products obtained after hydrolysis in DI water. Sample trapped in an air-tight syringes (Model: 1100 Gastight syringes, Hamilton Company, Reno, NV) and then attached to the gas sampling port on the side of the GC. 


\subsection{Results and Discussion}

The experimental conditions and results obtained from experiments completed on the carbothermic synthesis of calcium carbide for this exploratory work are presented in this chapter. The results discussed are for the reactions completed in both the microwave and conventional furnaces. The lime and carbonaceous material (coal, graphite and coal char) precursors used in these experiments were varied as explained in chapter 3 and the effect of the heating method, material chemistries and morphologies on the reaction were investigated. As outlined in the objective statement, the principal parameters influencing the reaction mechanism were determined from experimental observations of weight loss and carbide formed analysis after the reaction was completed. Some of the formulas used in obtaining these quantities from the experimental data, as well as detailed sample calculations, for results obtained by the GC are presented in Appendix I.

\subsection{Heat Energy Transfer Study}

With reference to the generally accepted reaction for the formation of calcium carbide, represented here as reaction II, to form one mole of calcium carbide, the energy required is approximately $464.8 \mathrm{~kJ}$. The reaction rate for endothermic gas-solid reactions is affected by heat transfer from the furnace to the reactants [2]. To determine whether the total energy transferred to the sample particles measure up with the heat absorbed by the samples placed at the furnace hot-zone region during a run, a separate study was conducted in which the effects of pellet thickness (for a given pellet diameter) on the heating rate of the lime and carbon mixture was examined. Pellet thickness from $1 \mathrm{~mm}$ to $4 \mathrm{~mm}$ where exposed to heat inside the furnace. The data shown in figure 4.1 was generated with a 2D conduction heat transfer code in Matlab. It was 
determined that for a furnace heating rate of $3{ }^{\circ} \mathrm{C}$ per minute in a conventional furnace, the actual temperature profiles of the pellets followed closely the temperature profile (as shown in figure 4.1) of the furnace with negligible variation due to differences in pellet thickness. This study was however not necessary for reactions completed using microwave heating because the temperature were taken from the sample surface with the pyrometer. The emissivity was calibrated and manually adjusted for accurate off-surface temperature measurements.

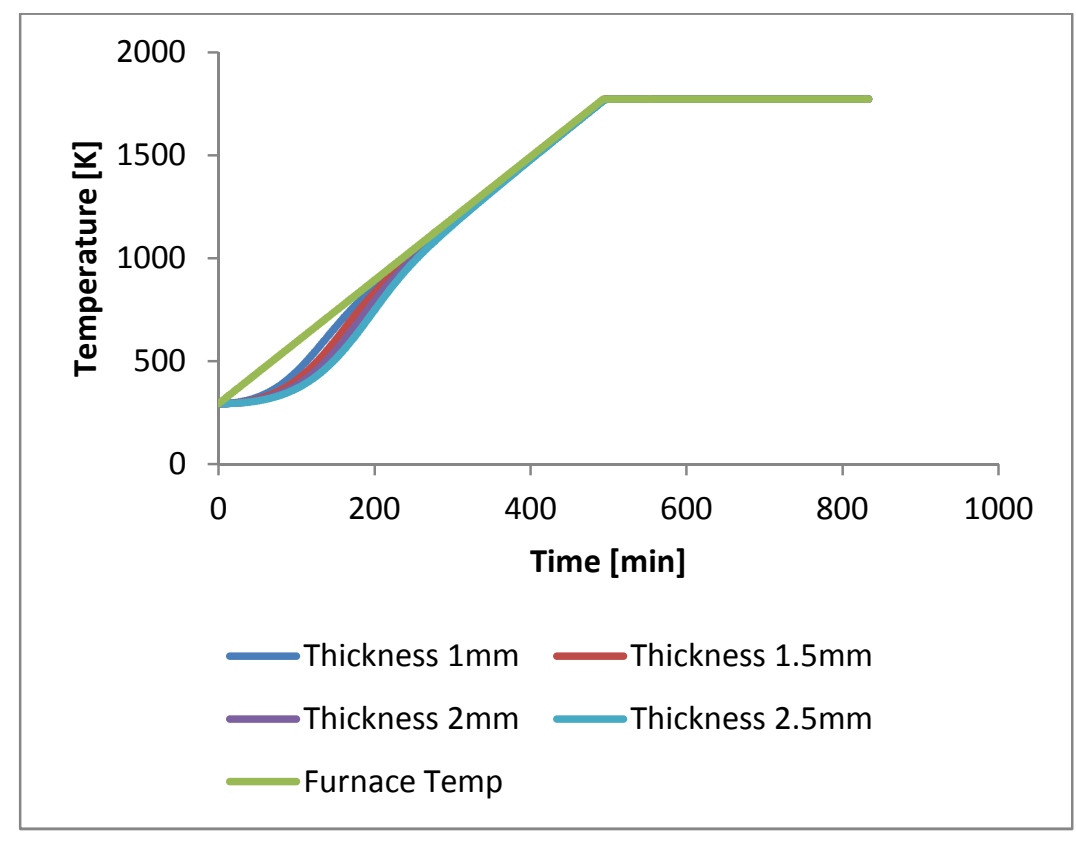

Figure 4.1: Temperature measurement profile for various sample thickness.

\subsection{Reaction Kinetics, Model Parameters and Gravimetric Measurement}

Experimental studies are described theoretically and mathematically by a model and translated into rate equation. The mechanistic bases for modeling solid-state kinetic studies are nucleation, geometrical contraction, diffusion, and reaction order [56]. Table 4.1 summarizes existing models from literatures. 
The general representation of rate equation is:

$$
\frac{\mathrm{d} \alpha}{\mathrm{dt}}=\mathrm{Ae}^{-\left(\mathrm{E}_{\mathrm{a}} / \mathrm{RT}\right)} \mathrm{f}(\alpha)
$$

Where, $\mathrm{A}$ is the pre-exponential (frequency) factor, $\mathrm{E}_{\mathrm{a}}$ is the activation energy, $\mathrm{T}$ is absolute temperature, $\mathrm{R}$ is the gas constant, $\mathrm{f}(\alpha)$ is the differential form of the reaction model, and $\alpha$ is the conversion [56].

Table 4.1: Solid-State Rate and Integral Expressions for Different Reaction Models [56]

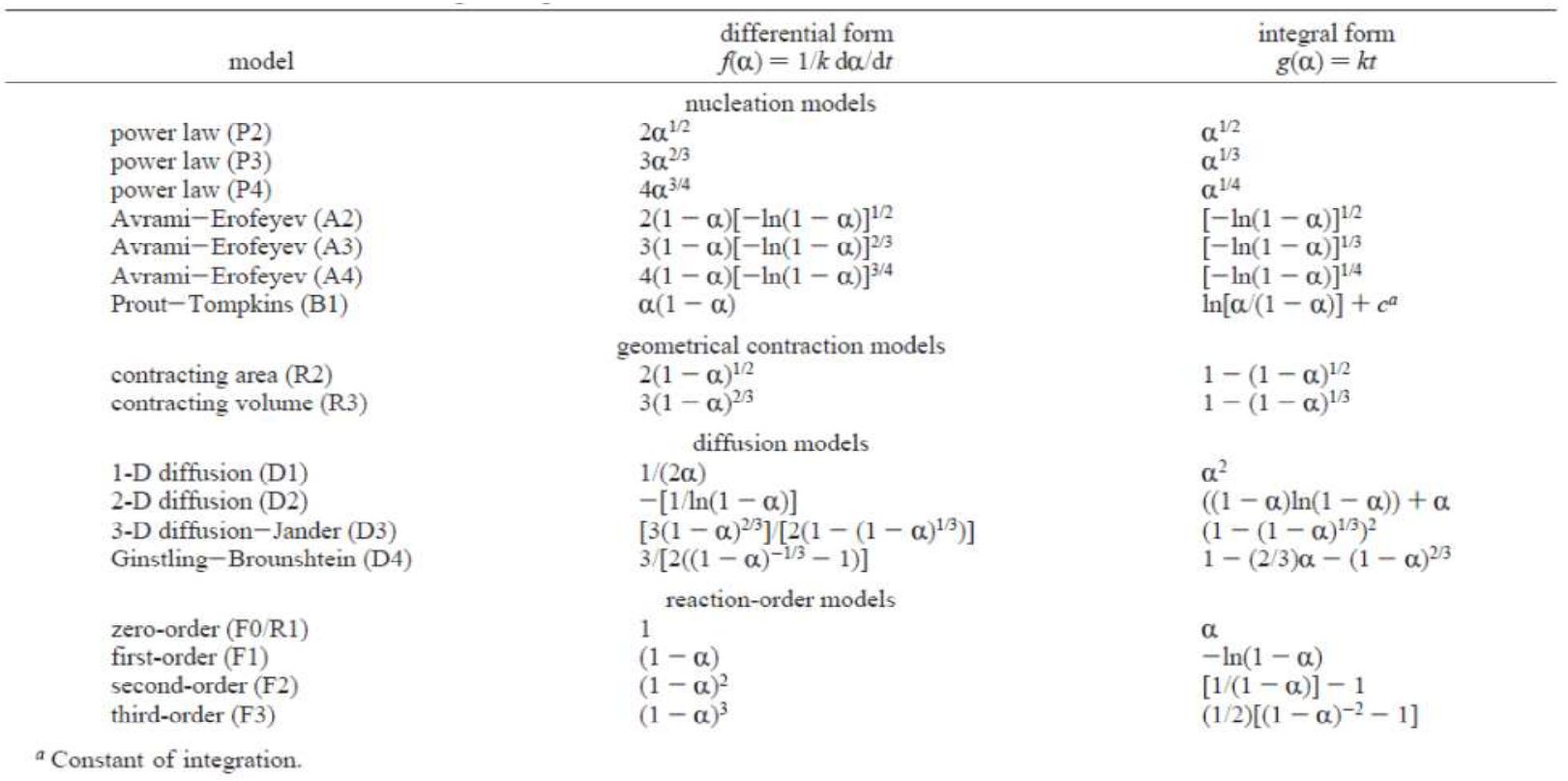

In this work, reaction kinetics in the solid-state was studied by powder X-ray diffraction (PXRD) and gas chromatographic analytical methods. The measured parameter was transformed into a conversion fraction that was used in the kinetic equations. The apparent conversion (AC) or degree of reaction at various reaction exposure times obtained at different temperature levels were calculated using equation 4.2.

$$
\mathrm{AC}=\frac{W_{i i}-W_{i}}{W_{0}}
$$


Where,

$\mathrm{W}_{\mathrm{i}}=$ Sample weight loss before exposure time

$\mathrm{W}_{\mathrm{ii}}=$ Sample weight loss after exposure time

$\mathrm{W}_{0}=$ Maximum theoretical weight loss calculated from the generally accepted reaction II

The percent calcium oxide used to form the carbide was therefore estimated using the relationship:

$$
\% \mathrm{CaO} \text { Conversion }=\left(\frac{N_{\mathrm{CaC}_{2}}}{N_{C a}}\right) \times 100
$$

Where,

$\mathrm{N}_{\mathrm{CaC}_{2}}=$ number of moles of $\mathrm{CaC}_{2} ; \mathrm{N}_{\mathrm{CaC}_{2}} \equiv \mathrm{N}_{\mathrm{C}_{2} \mathrm{H}_{2}}$

$\mathrm{N}_{\mathrm{Ca}}=$ number of moles of $\mathrm{Ca}$; equivalent to that of $\mathrm{CaO}$ before reaction.

It is assumed that calcium found in the final product existed as $\mathrm{CaO}$ before the commencement of the reaction, and the amount in percent of the total $\mathrm{Ca}$ that existed as $\mathrm{CaC}_{2}$ in the sample was represented at the percent conversion to $\mathrm{CaC}_{2}$. .

\subsection{Carbo-thermic Reduction Via Conventional Heating Technique}

\subsection{1 $\mathrm{Ca}(\mathrm{OH})_{2}$ Impurities}

Initial exploratory results obtained from reactions completed between $1500-1800^{\circ} \mathrm{C}$ recorded high weight losses. Preliminary investigations from literature suggest that the calcium carbide decomposition at higher temperature and calcium vaporization might be contributing to the weight loss. However, it is also possible the specimen would either gain weight or transfer mass 
to another form during the sample preparation stages before reaction even takes place despite the pre-treatments carried out on the starting materials before ball milling. If this is the case, calcium oxide might have picked up moisture and transformed into calcium hydroxide at room temperature at the ball-milling, sieving or pellet pressing stages.

To verify this, two sets of experiments were conducted with and without known $\mathrm{Ca}(\mathrm{OH})_{2}$ impurities, to determine the contribution of this inclusion in the reactants mixture. Figure 4.2 is an XRD confirmation of about 24\% moisture picked up and present in the mixture before reaction take place.

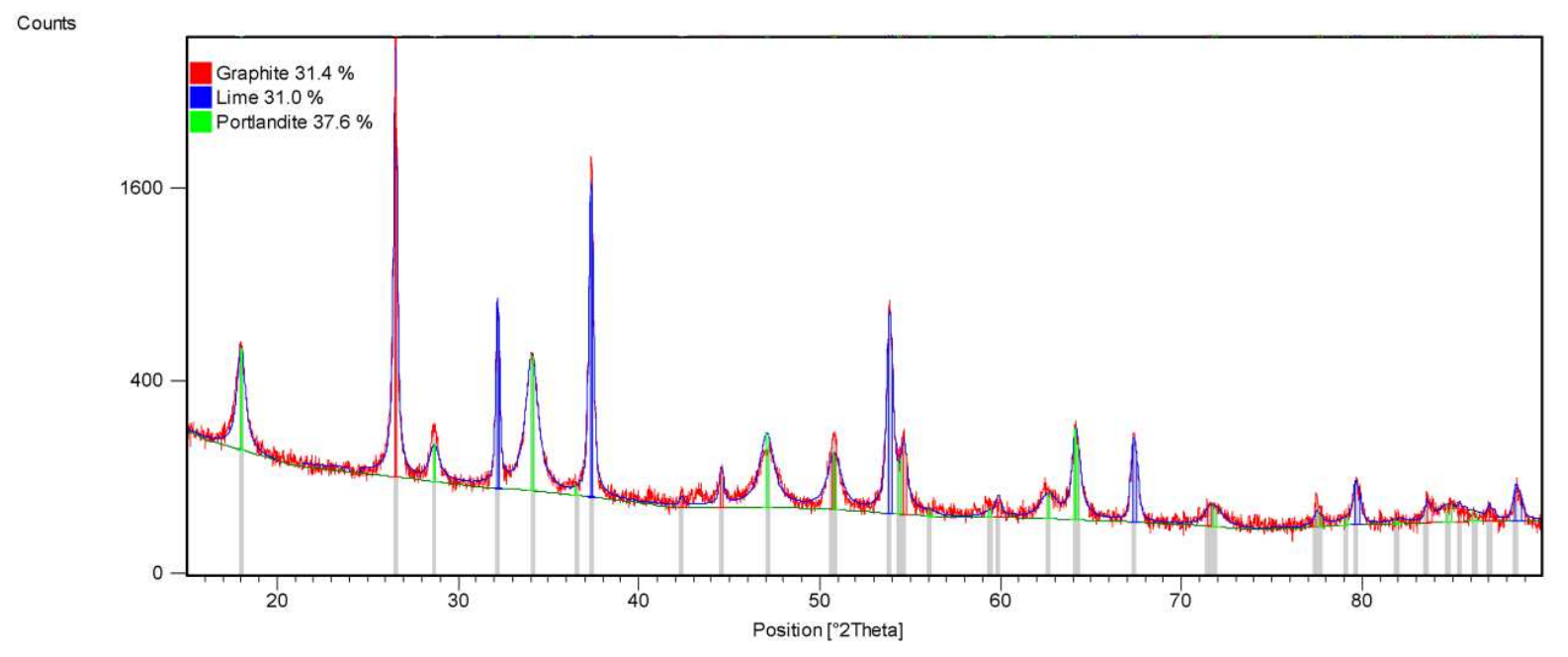

Figure 4.2: XRD pattern for sample (pre-treated before ball milling) with $\mathrm{Ca}(\mathrm{OH})_{2}$ impurities. Mass compositions of phases present were quantified by Rietveld technique and the calculated amount of moisture picked up is $24.29 \%$ of initial mass. The bottom parts of the graph shows the residue which is used to determine the goodness of the fit.

After a couple of runs, about $20 \%$ additional weight loss were recorded due to this inclusion. To address this new development, an additional heat treatment step was introduced before pellet pressing. Heat treatment here is not the same as that carried out before ball-milling the starting material mixture; it is usually completed before introducing the sample into the furnace. The heat treatment program used is provided in table 4.2 and was henceforth used for subsequent 
reactions. The pre-sample introduction into the furnace additional heat treatment step became necessary when $\mathrm{Ca}(\mathrm{OH})_{2}$ presence was found in the already heat-treated powder mixture ball milled and kept inside the glove box.

Table 4.2: Sample pre-treatment program per run in the furnace.

\begin{tabular}{|c|c|c|}
\hline Heating & Holding time & Cooling \\
\hline $900\left({ }^{\circ} \mathrm{C}\right), 3^{\circ} \mathrm{C} / \mathrm{min}$ & $4 \mathrm{hrs}$ & $900\left({ }^{\circ} \mathrm{C}\right), 3^{\circ} \mathrm{C} / \mathrm{min}$ \\
\hline
\end{tabular}

As seen in figure 4.3, calcium hydroxide impurities were completely eliminated and the Rietveld quantification of the mixture before run, matches with the charge composition of the $1 / 3$ mole ratio of calcium oxide and carbon mixture according to reaction II.

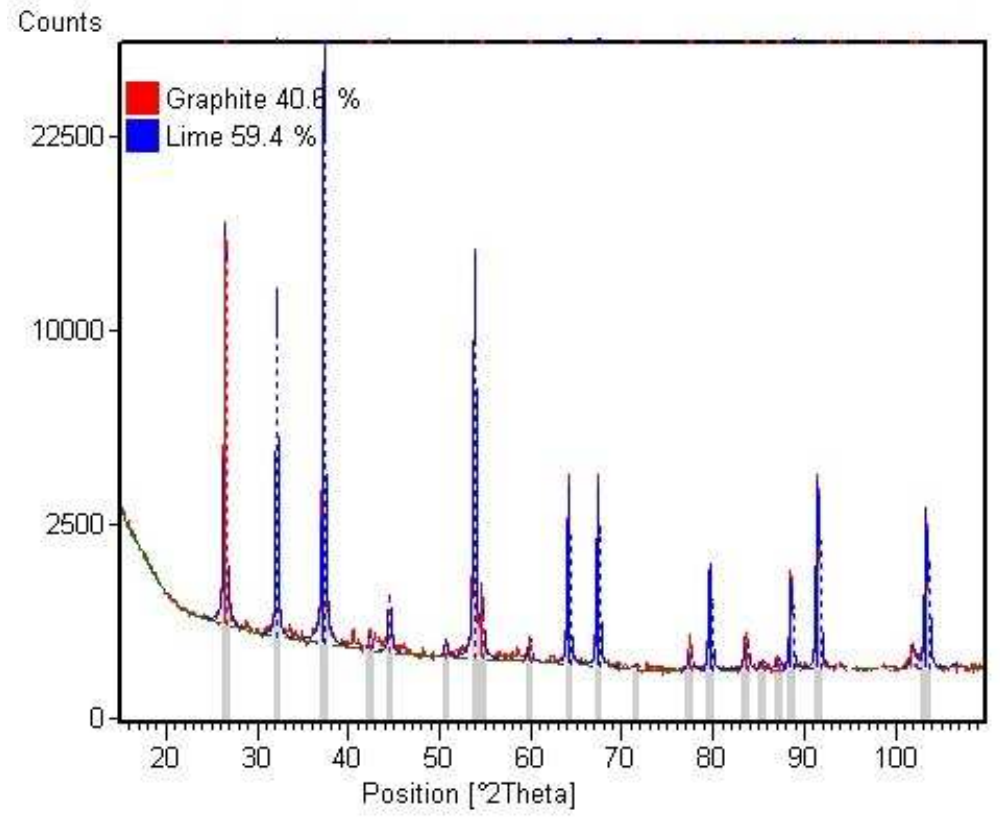

Figure 4.3: Rietveld quantification of heat-treated sample mixture before introduction into the furnace. 


\subsubsection{Influence of Mold and Tube Materials}

Preliminary experiments were conducted using alumina and zirconia as the materials for the boat crucibles and an alumina tube material described in chapter 3. However, it was unsatisfactory because of the reaction of the mold and tube materials with escaping calcium vapor at higher reaction temperatures. This is evident due also to the difficulty encountered in removing samples from the crucible and the crucible from the tube due to adhesion caused by the extensive deposition of the calcium deposits onto the wall of the crucible and tube. These reactions are possible thermodynamically within a temperature range of 900 to $1200^{\circ} \mathrm{C}$ (46). $\mathrm{Ca}_{12} \mathrm{Al}_{14} \mathrm{~F}_{2} \mathrm{O}_{32}$ and $\mathrm{Ca}_{5} \mathrm{Al}_{6} \mathrm{O}_{14}$ compounds were identified as contaminants in reaction completed using alumina crucible. To show that the vaporizing calcium escape, the exit carrier gas (in this work argon) that is usually bubbled through D.I. water at the exit end of the tube was collected and analyzed. This gas is collected over some of the experiments conducted at $1500^{\circ} \mathrm{C}$ for 60 minute isothermal hold and then analyzed. Results obtained were compared to pure distilled water and a significant amount of $\mathrm{Ca}$ was found present. In distilled water, less than $0.1 \mathrm{mg} / \mathrm{L}$ of calcium was found present while $1.92 \mathrm{mg} / \mathrm{L}$ of Calcium detected in the collected exit water analyzed. The detection limit for the method used is $0.1 \mathrm{mg} / \mathrm{L}$.

However, the use of a graphite crucible in this kind of environment yielded positive results. So far, it was possible to continue a series of experiments using one graphite crucible with no loss recorded due to chemical interaction. All subsequent experiments were therefore completed in the graphite mold. It is not the same with the tube as the methods introduced to trap some of the escaping vaporized calcium inside the crucible; in order to extend the service life of the tube, seem not to have worked out as expected, it only managed to reduce this effect minimally. 


\subsubsection{Influence of Mold Configuration}

The reaction container configuration (mold with lid and without lid) effect on the weight loss was investigated inside the tube furnace at $1500^{\circ} \mathrm{C}$ with a 60 minute isothermal hold. Figure 4.4

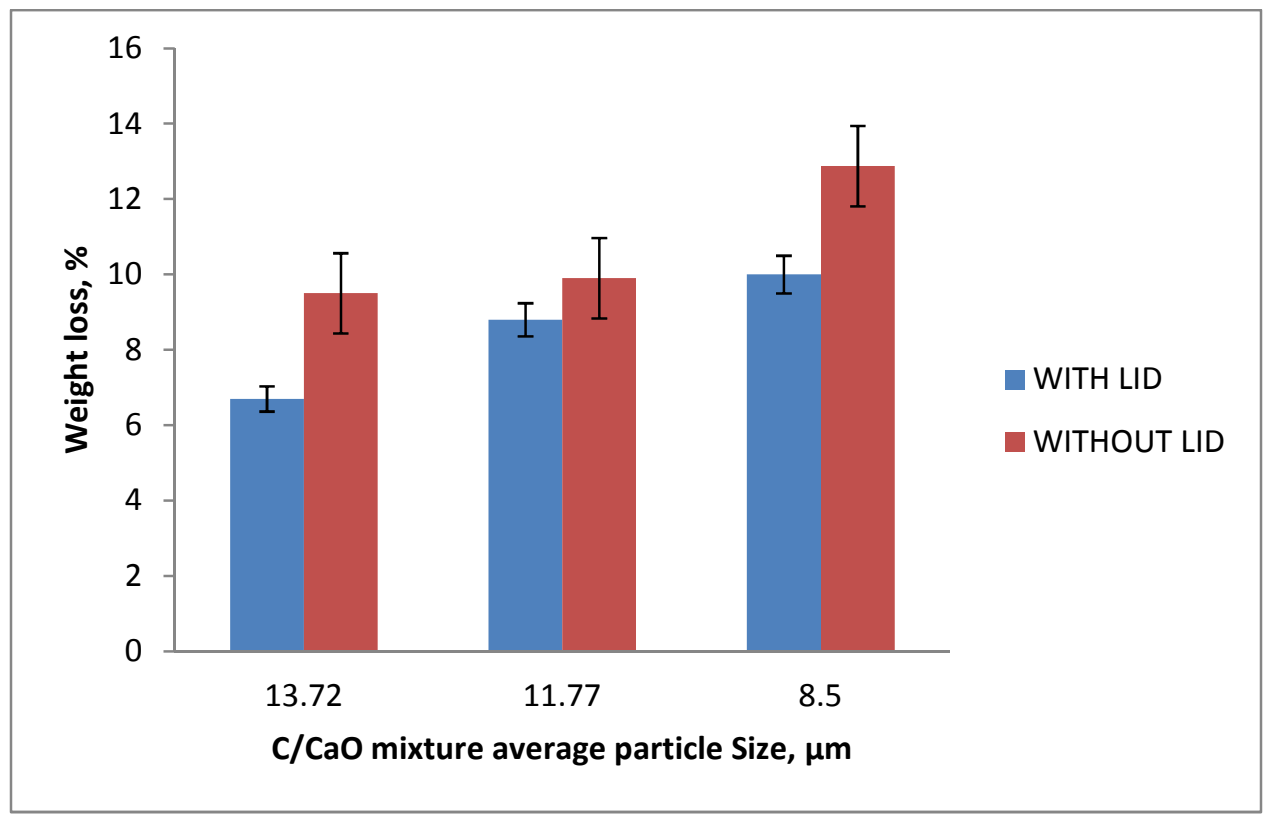

Figure 4.4: Influence of crucible configuration and particle size as a function of temperature on the weight loss of reaction completed. Graphite crucible with and without lids were used in this study.

shows a table for the various average particle sizes of starting materials used. The data shows that the use of a lid with the crucible resulted in lower weight losses. One possible explanation for this is that the lid may have increased the partial pressure of $\mathrm{CO}$ within the crucible, and as a result, the reactivity of the sample decreased. Clearly, there is a significant effect in the degree of reaction. As the $\mathrm{CO}$ concentration increases within the crucible, the rate of formation is limited. This trend is seen across the various particle sizes of $\mathrm{C} / \mathrm{CaO}$ mixture investigated. 


\subsubsection{Investigation of Reaction Variables' Influence}

Experimental results obtained from reacting a 1/3 mixture (on a mole basis) of lime and carbon was used to study the kinetics of the formation of calcium carbide.

The formation of calcium carbide from carbon and lime is governed by multi-step reactions II, III, IV and $\mathrm{V}$ as agreed by most previous works. It is also widely accepted that once $\mathrm{CaC}_{2}$ is formed, $\mathrm{CaC}_{2}$ can either dissociate into calcium and carbon via reaction (IV), $\mathrm{Ca}(\mathrm{g})+2 \mathrm{C} \rightarrow$ $\mathrm{CaC}_{2}(\mathrm{~s})$ or react with $\mathrm{CaO}(\mathrm{s})$ per reaction $(\mathrm{V}), \mathrm{CaC}_{2}(\mathrm{~s})+2 \mathrm{CaO}(\mathrm{s}) \rightarrow 3 \mathrm{Ca}(\mathrm{g})+2 \mathrm{CO}(\mathrm{g})$.

The reaction path in this work followed a multi-step mechanism. Mukaibo et al. [43] found the procession of reaction $\mathrm{III}, \mathrm{CaO}+\mathrm{C} \rightarrow \mathrm{Ca}(\mathrm{g})+\mathrm{CO}$ above $1150{ }^{\circ} \mathrm{C}$, while reaction $\mathrm{IV}, \mathrm{Ca}(\mathrm{g})+$ $2 \mathrm{C} \rightarrow \mathrm{CaC}_{2}$ did not take place below $1300^{\circ} \mathrm{C}$. The Tammann temperature (approximately half the melting point in kelvin) for $\mathrm{CaO}$ is $1158^{\circ} \mathrm{C}$. At this temperature, the mobility and reactivity of the molecules in the bulk of $\mathrm{CaO}$ become appreciable. In our experiment at $1500^{\circ} \mathrm{C}$, we carried out the heating of a $\mathrm{C} / \mathrm{CaO}$ mixture, calcium metal evaporated from the sample were found from the analysis of DI water collected at the exit end of the furnace after the run-the carrier gas is usually bubbled through this DI water at the exit end of the furnace. As explained before, $1.92 \mathrm{mg} / \mathrm{L}$ of Calcium was detected in the sample exit water analyzed as against $>0.1 \mathrm{mg} / \mathrm{L}$ present in the control sample D.I. water. The detection limit for the method used was $0.1 \mathrm{mg} / \mathrm{L}$.

Also, XPS surface analysis instrument which can provide elemental and chemical information about the surface region (first 1- 30 monolayers) of nearly any solid material (see figure 4.5) found an appreciable presence of calcium and calcium oxide as carbon decreases across the depth probed. The essence of this analysis is to monitor the distribution of the constituent 
materials and quantify phases present after the reaction of the pressed pellet of $\mathrm{C} / \mathrm{CaO}$ mixture at $1500^{\circ} \mathrm{C}$ for 60 minutes. Figure 4.5 shows the depth profile analysis data collected for the reacted sample $\left(1 / 3\right.$ carbon and calcium oxide mixture reacted at $1500^{\circ} \mathrm{C}$ for 60 minutes $)$.

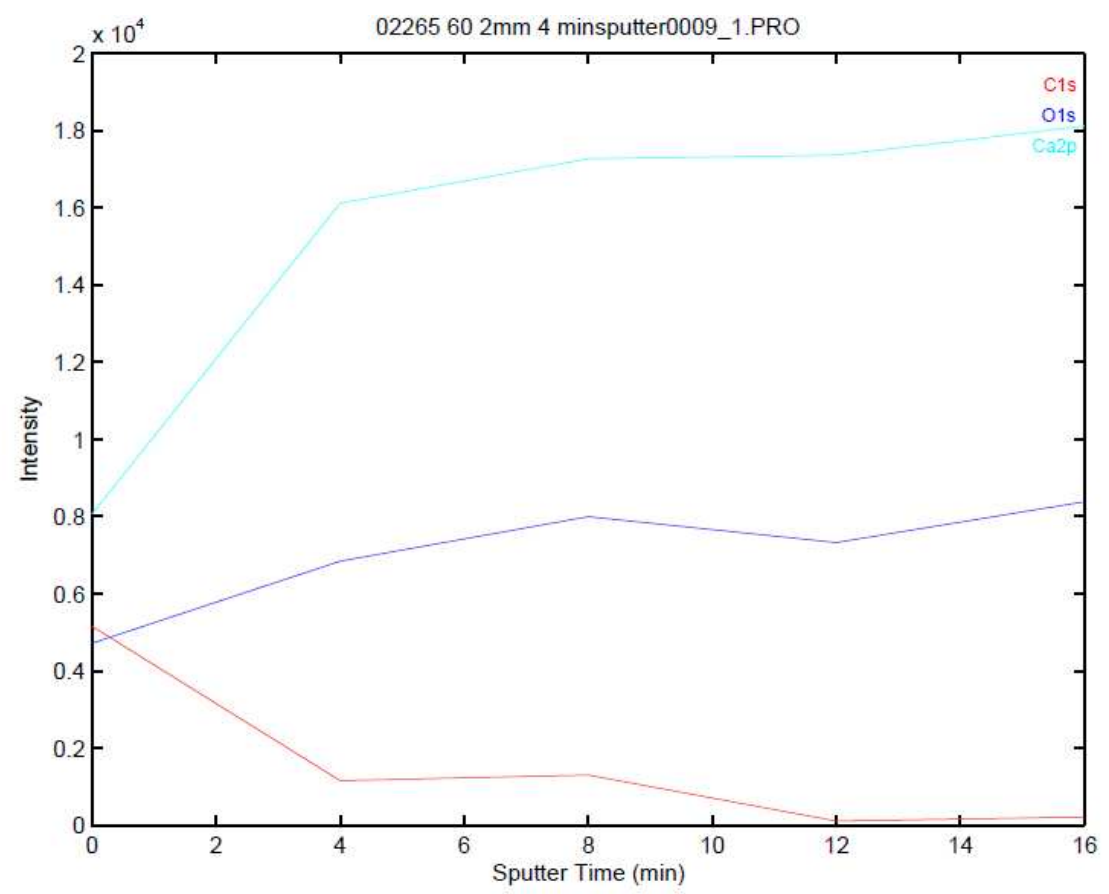

Figure 4.5: XPS surface analysis technique depth profile scan for $1 / 3$ carbon and calcium oxide mixture reacted at $1500^{\circ} \mathrm{C}$ for 60 minutes. The surface was sputtered to make sure that carbon detected is from the sample, not contaminant. Specifications:

- Detection limits (all elements except $\mathrm{H}$ ): $\sim 0.01$ monolayer, or $\sim 0.1 \%$ bulk

- Measurement depth: 10 - $50 \AA$; area: 0.2 - 1 mm dia.

- Sample size: $0.1 \mathrm{~mm}$ to $76.2 \mathrm{~mm}$ dia. $\mathrm{x} 25.4 \mathrm{~mm}$ thick

- Spectrometer: Monochromatized Al (ka) Source; Vacuum 5 x 10E(-10) torr

The surface was sputtered to make sure that carbon detected is not surface contaminant. It can be seen that the concentration of calcium element close to the surface decreases; some of these are the ones displaced towards the exit end of the tube as detected by the water analysis test. An estimated 6\% calcium carbide was formed using the XPS quantification technique. Meanwhile, 
GC analysis estimation of calcium carbide at this temperature and exposure time was $10 \%$.

Reaction III, $\mathrm{CaO}+\mathrm{C} \rightarrow \mathrm{Ca}(\mathrm{g})+\mathrm{CO}$ seems to be dominant at this temperature.

Table 4.3 shows the extent of reaction over time for three different carbon types reacted separately with $\mathrm{CaO}$. The presence of volatile materials seems to play a vital role according to the data shown in table 4.3; graphite has the least amount. Coal is more volatile and as such, has the highest weight loss both in powder and pellet forms. Coal is filled with other volatile materials that decompose at high temperature and this might have contributed to the weight loss reported here.

Table 4.3: Weight loss as a function temperature for various carbon sources used. All experiments done at 60 minute hold time and the reacted samples placed in zirconia combustion crucibles.

\begin{tabular}{lllllll}
\hline & \multicolumn{3}{c}{ Pellet } & \multicolumn{2}{c}{ Powder } \\
\cline { 2 - 6 } & Graphite & Coal & Coal-Char & Graphite & Coal & Coal-Char \\
\hline $1500^{\circ} \mathrm{C}$ & 10.49 & 39.3 & 35.36 & 17.00 & 46.2 & 43.94 \\
\hline
\end{tabular}

In the work of Mukaibo and Yamanaka, the rate of reaction for reactions III and IV is directly proportional to the area of contact between $\mathrm{CaO}$ and $\mathrm{C}$ [43]. However, as seen in table 4.4, the degree of reaction (in terms of weight loss) was reduced as the surface contacts between reactants were increased. Influence of particle size and applied compaction load on the weight loss at $1500^{\circ} \mathrm{C}$ and $860 \mathrm{~min}$ reaction time (for a $12.77 \mathrm{~mm}$ diameter die; $0.55 \mathrm{~g}$ average mass; 1 ksi-7 ksi compaction pressure) showed that weight loss decreased slightly with increased compaction load (green density) and inversely with particle size of the starting materials. As the temperature increases, these effects become much more significant. 
Table 4.4: Influence of particle size and applied pellets $(12.77 \mathrm{~mm}$ diameter, $2.5 \mathrm{~mm}$ thickness, $0.55 \mathrm{~g}$ average mass of powder granules) compaction load on the weight loss at reaction temperature $1500^{\circ} \mathrm{C}$ and 460 minutes reaction time in argon environment.

\begin{tabular}{cccccc} 
Particle Size Distribution, & \multicolumn{5}{c}{ \% Weight Loss } \\
${ }(\mu \mathrm{m}) }$ & 1 & 2 & 3 & 5 & 7 \\
\cline { 2 - 6 } & 12.88 & 12.58 & 12.20 & 12.14 \\
\cline { 2 - 6 } & 7.5 & 7.1 & 6.28 & 5.9 & 5.87 \\
\hline 13.5 & & & \multicolumn{5}{c}{ Pellets Compaction Pressure (kPsi) } \\
\hline
\end{tabular}

Lower $\mathrm{CaO}$ size provides higher surface area for $\mathrm{Ca}$ loss and increased particle-particle contact for solid state reaction. Weight loss may have decreased with compaction due to less solid-gas interfaces (for sublimation) and more solid-solid interfaces (for reaction). The tortuosity may have increased which may decrease mass diffusion of Ca vapor out of the structure (increasing local vapor pressure, which would decrease sublimation rate) and the same would restrict the product $\mathrm{CO}$ out of the structure which locally references the reaction path.

The expectation for solid-solid surface compaction behavior was to have more yield as the reactants are in close proximity, however, this was not what happened. This is because of the restriction in solid-state mass transfer of calcium vapor and $\mathrm{CO}$ released possibly caused by formed $\mathrm{CaC}_{2}$ around reactants and trapped $\mathrm{CO}$. The $\mathrm{CO}$ being the lighter gas is expected to displace heavier vaporize $\mathrm{Ca}$, which means that $\mathrm{Ca}$ vapor diffuses in the gas form, adsorbs on the carbon surface, and then nucleates the desired phase in situ.

Dejun Shi et al. (47) investigated and confirmed restriction in movement of calcium vapor and $\mathrm{CO}$ released during carbide formation. This is possibly caused by formed $\mathrm{CaC}_{2}$ around reactants and trapped $\mathrm{CO}$. They showed this in their work by introducing potassium carbonate as an 
additive during the synthesis of calcium carbide to permit alternative transport route for contact between $\mathrm{CaO}$ and carbon, which was essential for the solid-solid reaction to increase yield of calcium carbide. The role of potassium carbonate was attributed to its melting and dissolution of $\mathrm{CaO}$ which enhanced the mass transfer of the latter to the carbon reactant. The carbonate would melt because it has a low melting temperature in none oxidizing atmosphere and the capillary action of the liquid pulled the particles closer (by reducing diffusion distances) and provided a means for liquid phase transport of $\mathrm{Ca}$ to the carbon interface (by dissolving in the carbonate solution since the liquid phase transport is faster compare to solid-state transport). A $10 \%$ increase in yield was reported for aided reaction in terms of permitting alternative transport route compared to the unaided reaction.

Figures 4.6, 4.7 as well as table 4.5, 4.6, respectively, show the effect of holding time and temperature on $\mathrm{CaC}_{2}$ formation via conventional heating. All curves of the weight loss against time in the temperature range $1500-1700^{\circ} \mathrm{C}$ are exponential. Figure 4.6 and 4.7 show the course of the reactions at four and three temperatures, respectively. It is expected that higher reaction temperatures or longer holding times will yield more $\mathrm{CaC}_{2}$. It is clear that the temperature has a profound influence on the rate of the reaction and densification. The density of the products decreases accordingly as the temperature and holding time is increased (table 4.5 and 4.6). This is believed to be due to the enhanced vapor pressure of calcium, which is a function of temperature and an enhanced diffusivity of the materials. As the temperature is raised, calcium particles melt and disperse such that the vapor pressure of calcium increases to a level that the porous $\mathrm{C} / \mathrm{CaO}$ matrix is penetrated to allow contact with carbon to form calcium carbide. 


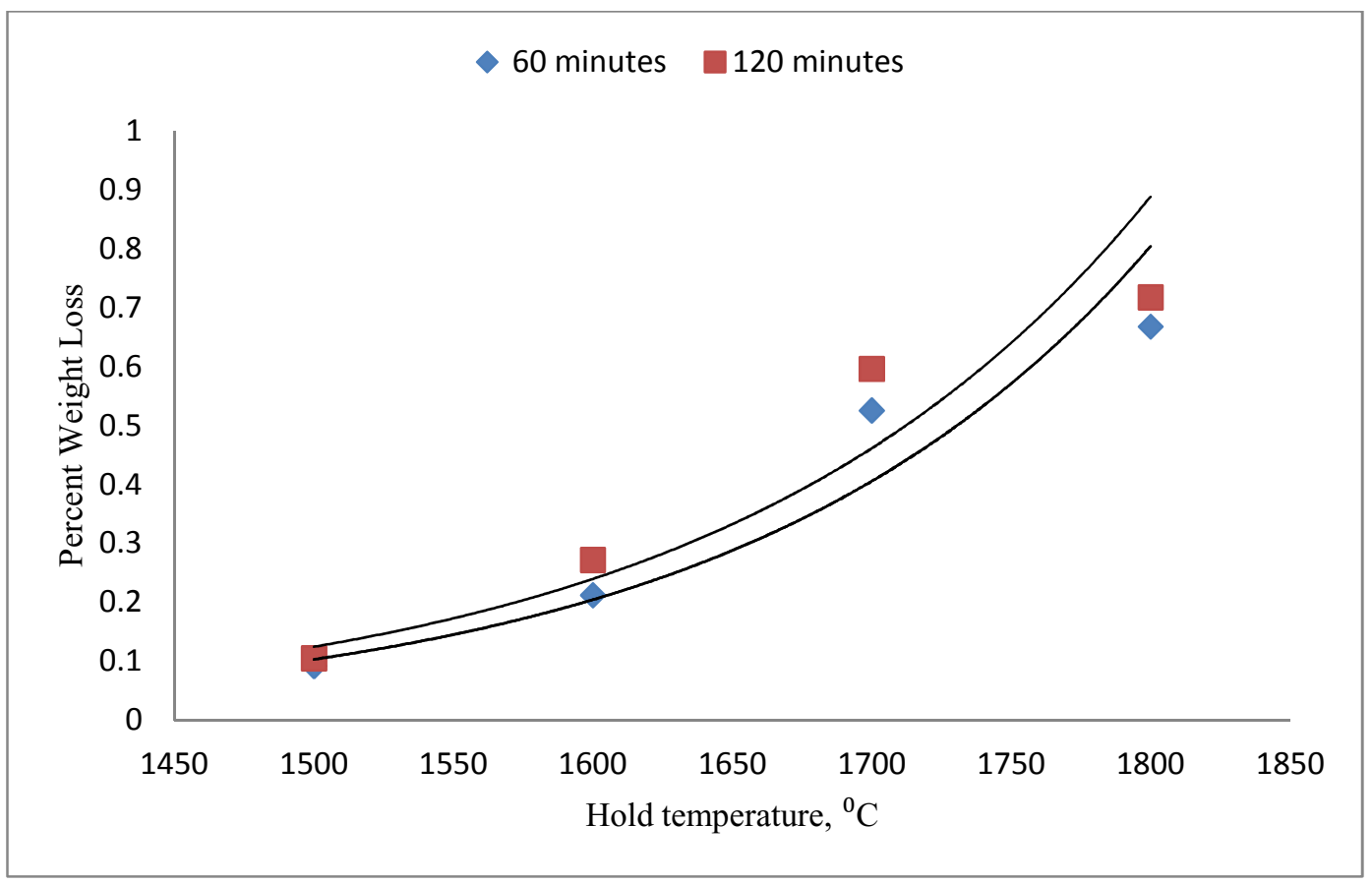

Figure 4.6: Weight loss as a function of temperature. All experiments were held at 60 and 120 minutes.

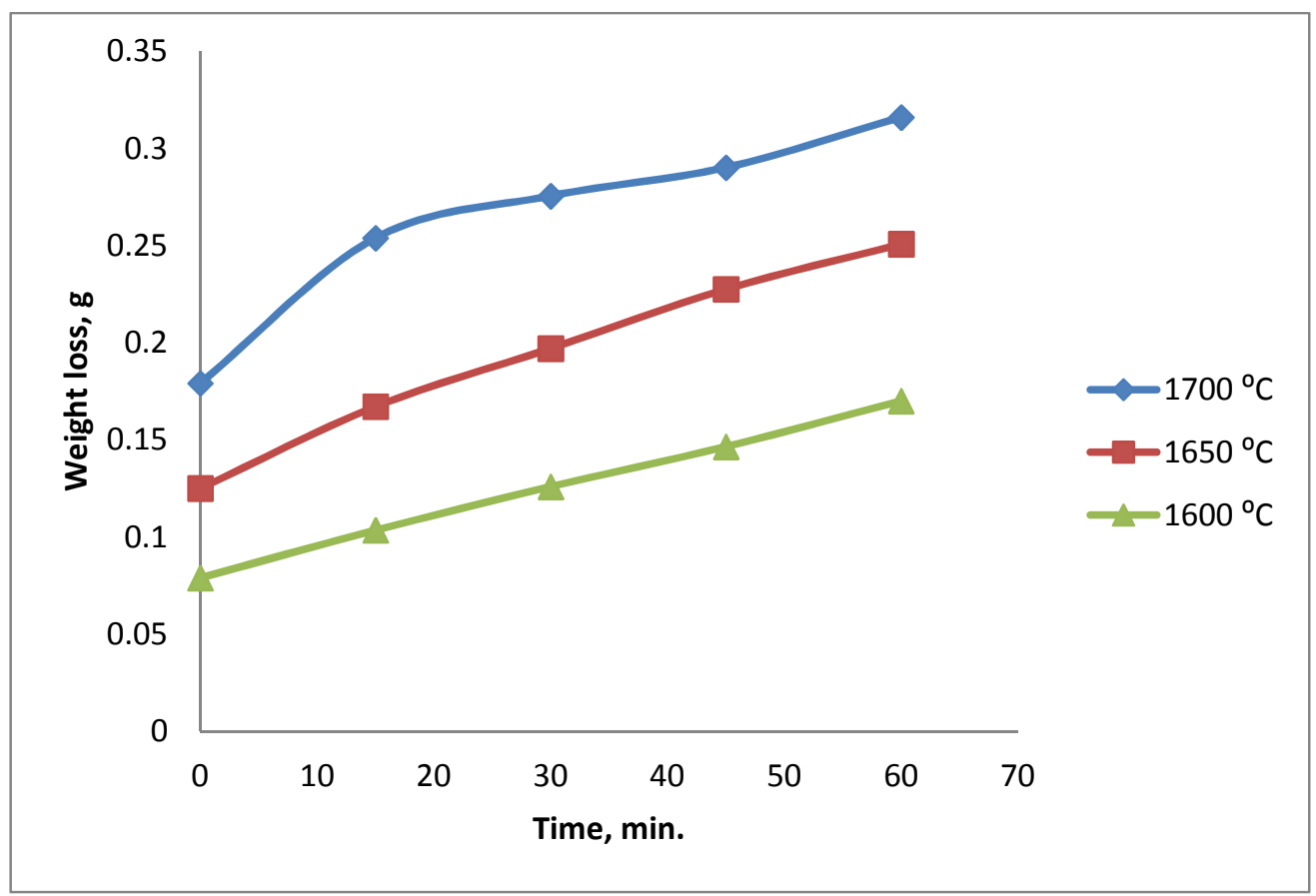

Figure 4.7 Relation between weight loss and exposure time. 
Table 4.5: Temperature study results in conventional furnace.

Reactants: Lime, Graphite (LGM)

Mold material and condition: Zirconia combustible boat crucible and without lid

Furnace type \& atmosphere: $1800^{\circ} \mathrm{C}$ RHTH, Argon gas, $0.25 \mathrm{bar}$

Sample mass and form: $0.55 \mathrm{~g}$, Pellet

Ball milling duration (hours): $24 \& 96$

Uni-axial compaction pressure: $1 \mathrm{kPsi}$.

\begin{tabular}{|c|c|c|c|c|c|c|}
\hline \multirow{2}{*}{\multicolumn{2}{|c|}{$\begin{array}{l}\text { Batch Code Temperature } \\
\text { Loss Apparent Conversion }\end{array}$}} & \multirow{2}{*}{ Time* } & \multirow[t]{2}{*}{ Green Density } & \multirow[t]{2}{*}{ Sintered Density } & \multirow[t]{2}{*}{ Percent } & \multirow[t]{2}{*}{ Weight } \\
\hline & & & & & & \\
\hline Name & ${ }^{\circ} \mathrm{C}$ & $\min$ & $\mathrm{g} / \mathrm{cm}^{3}$ & $\mathrm{~g} / \mathrm{cm}^{3}$ & $\mathrm{~T} \mathrm{D}^{* *}$ & $\%$ \\
\hline \multirow[t]{4}{*}{24 LGM } & 1500 & 560 & 1.91 & 1.73 & 77.93 & 10.49 \\
\hline & 1600 & 593 & 1.90 & 1.53 & 68.92 & 21.21 \\
\hline & 1700 & 627 & 1.91 & 1.01 & 45.50 & 52.56 \\
\hline & 1800 & 660 & 1.90 & 0.82 & 36.94 & 66.82 \\
\hline \multirow[t]{4}{*}{$24 \mathrm{LGM}$} & 1500 & 620 & 1.92 & 1.74 & 78.38 & 14.11 \\
\hline & 1600 & 653 & 1.92 & 1.35 & 60.81 & 28.21 \\
\hline & 1700 & 687 & 1.92 & 0.92 & 41.44 & 59.63 \\
\hline & 1800 & 720 & 1.91 & 0.75 & 33.68 & 71.77 \\
\hline \multirow[t]{4}{*}{96 LGM } & 1500 & 560 & 1.85 & 1.46 & 65.77 & 30.76 \\
\hline & 1600 & 593 & 1.80 & 1.17 & 52.90 & 46.71 \\
\hline & 1700 & 627 & 1.67 & 0.87 & 39.19 & 68.65 \\
\hline & 1800 & 660 & 1.85 & 0.66 & 29.73 & 79.84 \\
\hline
\end{tabular}

* Total time, $\mathrm{t}_{\text {react }}($ minutes $)=\mathrm{t}_{\text {hold }}+\left(\mathrm{t}_{\text {heat }} / 2\right)+\left(\mathrm{t}_{\text {cool }} / 2\right)$

** Based upon the theoretical density (TD) of the tetragonal $\mathrm{CaC}_{2} I\left(2.22 \mathrm{~g} / \mathrm{cm}^{3}\right)$

Table 4.6: Temperature study results in conventional furnace.

Reactants: Lime, Graphite (LGM)

Mold material and condition: Graphite square crucible and with lid

Furnace type \& atmosphere: $1800^{\circ} \mathrm{C}$ RHTH, Argon gas, $0.25 \mathrm{bar}$

Sample mass and form: $0.55 \mathrm{~g}$, Pellet (pre-introduction heat treatment)

Ball milling duration (hours): 504

Uni-axial compaction pressure: $1 \mathrm{kPsi}$.

\begin{tabular}{lccccccc}
\hline $\begin{array}{l}\text { Batch Code } \\
\text { Name }\end{array}$ & $\begin{array}{c}\text { Temperature } \\
{ }^{\circ} \mathrm{C}\end{array}$ & $\begin{array}{c}\text { Time* } \\
\mathrm{min}\end{array}$ & $\begin{array}{c}\text { Green Density } \\
\mathrm{g} / \mathrm{cm}^{3}\end{array}$ & $\begin{array}{c}\text { Sintered Density Percent } \\
\mathrm{g} / \mathrm{cm}^{3}\end{array}$ & $\begin{array}{c}\text { Weight Loss } \\
\text { T D** }\end{array}$ & $\begin{array}{c}\text { AC } \\
\%\end{array}$ \\
\hline 504 LGM & 1500 & 560 & 1.75 & 1.62 & 72.97 & 5.73 & 7.7 \\
& 1600 & 593 & 1.79 & 1.42 & 63.96 & 13.57 & 41.47 \\
& 1700 & 627 & 1.75 & 1.33 & 59.91 & 39.53 & 51.69 \\
\hline
\end{tabular}

AC-Apparent Conversion 
The apparent conversions based on weight loss curves in all experiments are parabolic (figure 4.8).

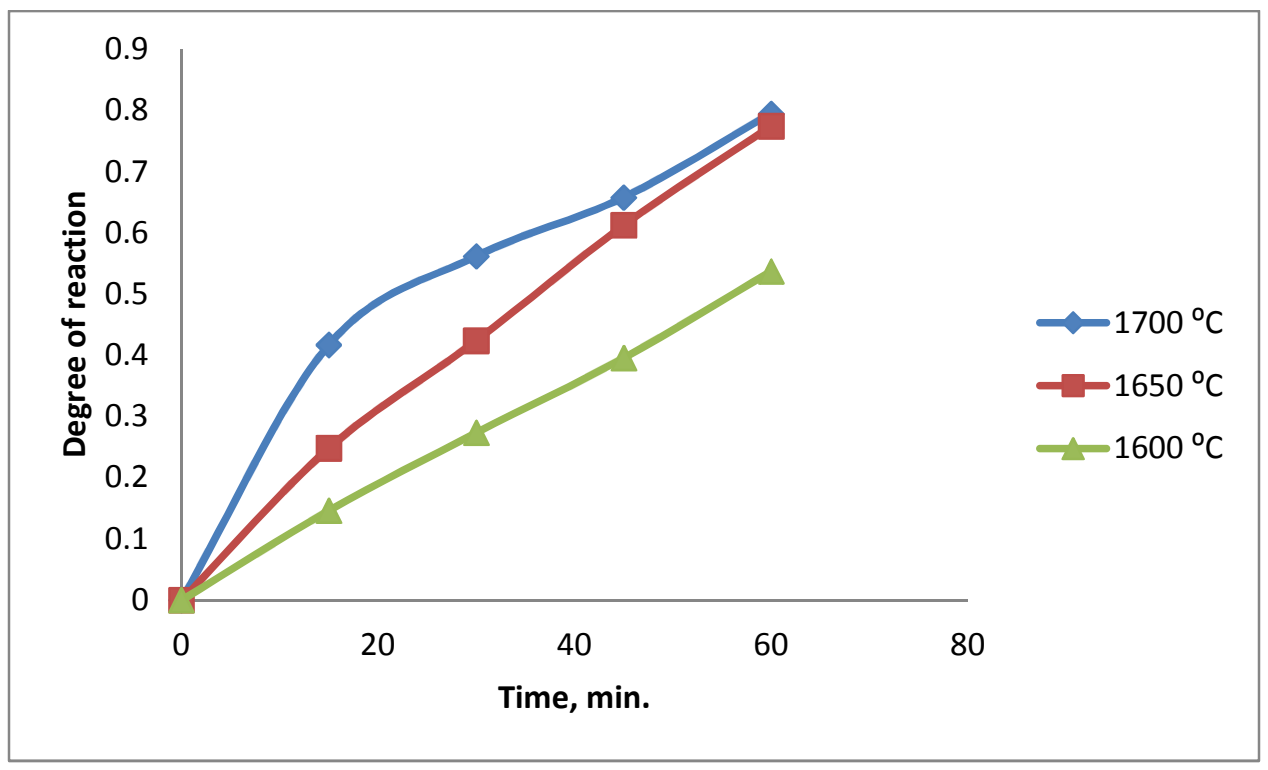

Figure 4.8 Relation between actual conversion and exposure time.

The conversion rate appears to increase exponentially with increasing temperature, which is an indication that the reaction followed a diffusion-controlled mechanism. The rate of solid state reaction when the diffusion process is rate-determining is expressed by the 3-dimentional parabolic model [56]:

$$
\text { Degree of reaction }(=\text { apparent conversion })=(1-\sqrt[3]{(1}-\alpha))^{2}=k t_{\text {react }}
$$

Where, $\alpha$ is the apparent conversion, $\mathrm{k}$ is the reaction rate constant and the total reaction time, $t_{\text {react }}$ is the time the sample was exposed to and it is given by:

$$
t_{\text {react }}=t_{\text {hold }}+\left(\frac{t_{\text {heat }}}{2}\right)+\left(\frac{t_{\text {cool }}}{2}\right)
$$

The three-dimensional diffusional (D3) model is based on the assumption of spherical solid particles. The parabolic kinetics given in equation (4.3) was therefore used to determine the reaction path and the curve shown in figure 4.9. The linearity of this curve indicates an apparent 
diffusion-controlled reaction. The slope of these lines was used to determine the apparent reaction constant, $\mathrm{k}$. The activation energy for the range of temperature considered in this work is $125.66 \mathrm{~kJ} / \mathrm{mol}$; it was obtained by plotting the logarithm apparent reaction kinetic constants shown in the data in figure 4.9 against the reciprocal of the absolute temperatures. The data shown in figure 4.10 were used to compute the activation energy. The slope of the curve was multiplied by the gas constant, $\mathrm{R}$ using the value $8.134 \mathrm{~kJ} / \mathrm{mol}$, to obtain the activation energy.

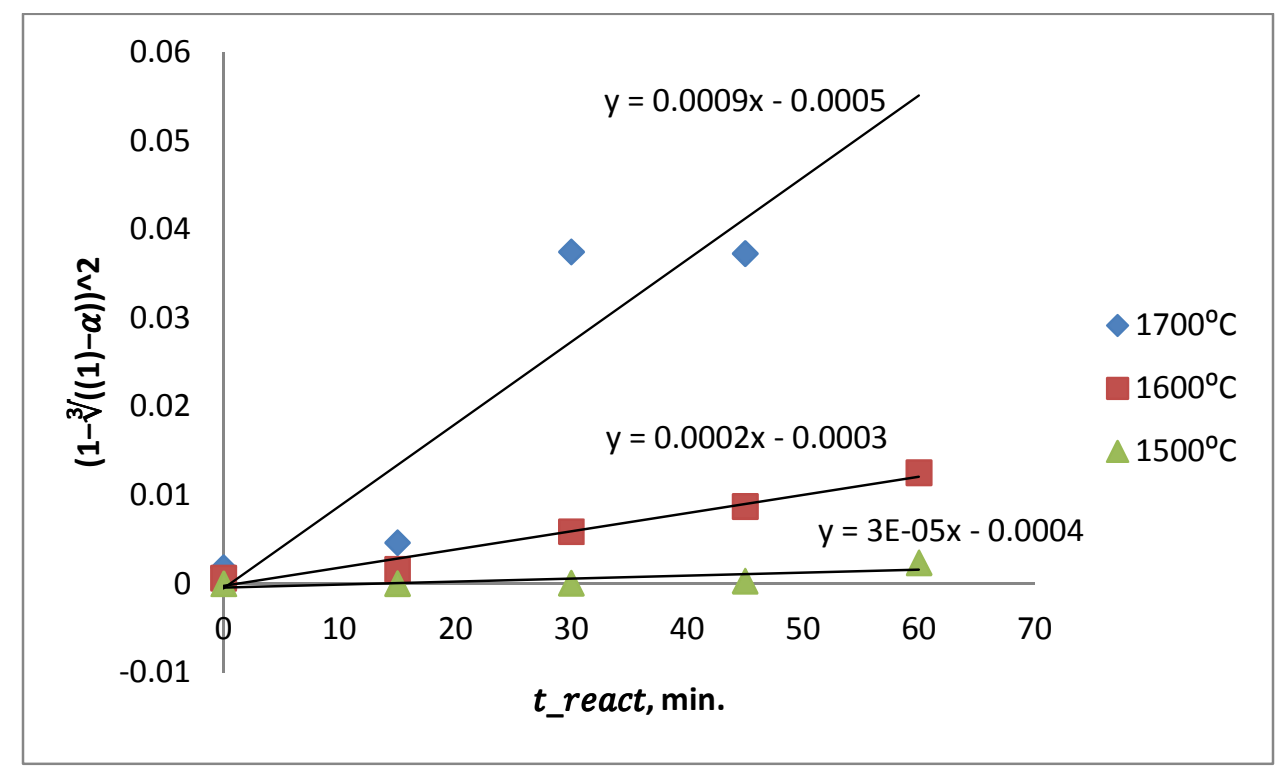

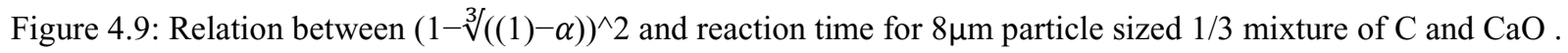




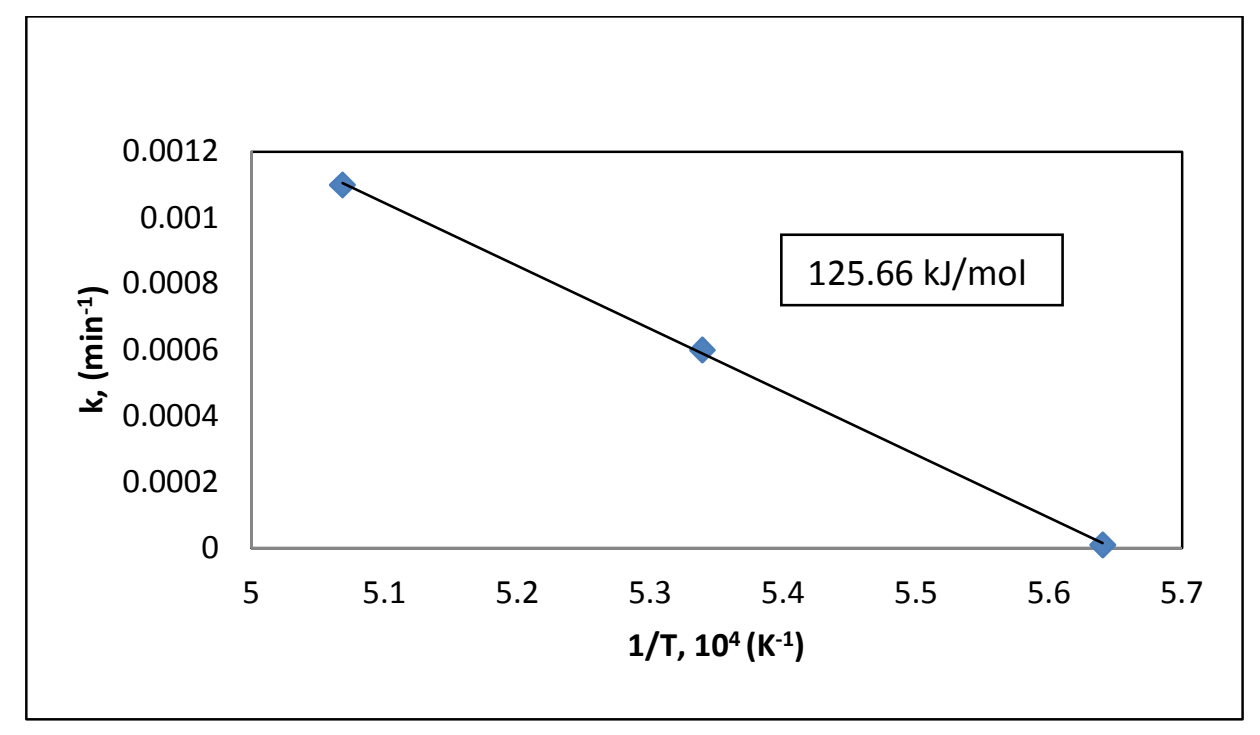

Figure 4.10: Relation between logarithm apparent reaction kinetic constants against the reciprocal of the reaction temperatures.

Tagawa and Sugawara reported $598 \mathrm{~kJ}$ mol-1 at a CO pressure of $50 \mathrm{mmHg}$ and $836 \mathrm{~kJ}$ mol-1 at a $\mathrm{CO}$ pressure of $100 \mathrm{mmHg}$. They reacted granules compressed from fine charcoal and $\mathrm{CaO}$ (at least 74 microns average particle sizes) with the $1 / 3$ molar stoichiometric ratio as defined by reaction II here, $\mathrm{CaO}+3 \mathrm{C} \rightarrow \mathrm{CaC} 2+\mathrm{CO}$, and experimentally fit their data with the diffusion model, $\left(1-(1-\alpha)^{1 / 3}\right)^{2}=\mathrm{kt}[8]$.

Guodong Li et al. also reported their apparent activation energy as about $456 \mathrm{~kJ}$ mol-1 at the lower temperature range and about $256 \mathrm{~kJ}$ mol-1 at the higher-temperature range $(20,140,250$ and 680 microns average particle sizes and bituminous coke as carbon source). They attributed the difference in activation energies to minimal $\mathrm{CO}$ pressure due to the constant purging by Argon [53].

Reduced activation energy for high temperature is usually attributed to presence of $\mathrm{CaC}_{2} \cdot \mathrm{CaO}$ eutectics at temperature greater than $1700^{\circ} \mathrm{C}$ for formation of calcium carbide. The break point at $1640^{\circ} \mathrm{C}$ seems to suggest so here, even though studies by both chemical and x-ray diffraction of 
the reactions completed (Figures $4.11,4.12,4.13,4.14$ and 4.15 ) did not identify any intermediate phase. In figure 4.11 , calcium carbide formed was identified at $1800^{\circ} \mathrm{C}$ with no evidence of any intermediate phases other than unreacted graphite, graphite from carbide that dissolved at higher temperature, calcium oxide and calcium hydroxide. The same observation from figures 4.12-15 show no evidence of any intermediate phase $(\mathrm{s})\left(\mathrm{CaC} \cdot \mathrm{CaO} / \mathrm{CaC}{ }_{2} \cdot \mathrm{CaO}\right)$ but a typical carbide phase that is very difficult to quantify particle size and with no typical shapes of crystals [57] detected. In figure 4.15, the dominant phases were plate-like carbon and calcium oxide, which is an indication of about $6-10 \%$ carbide formed confirmed by XPS, GC and XRD quantification technique used in this work.

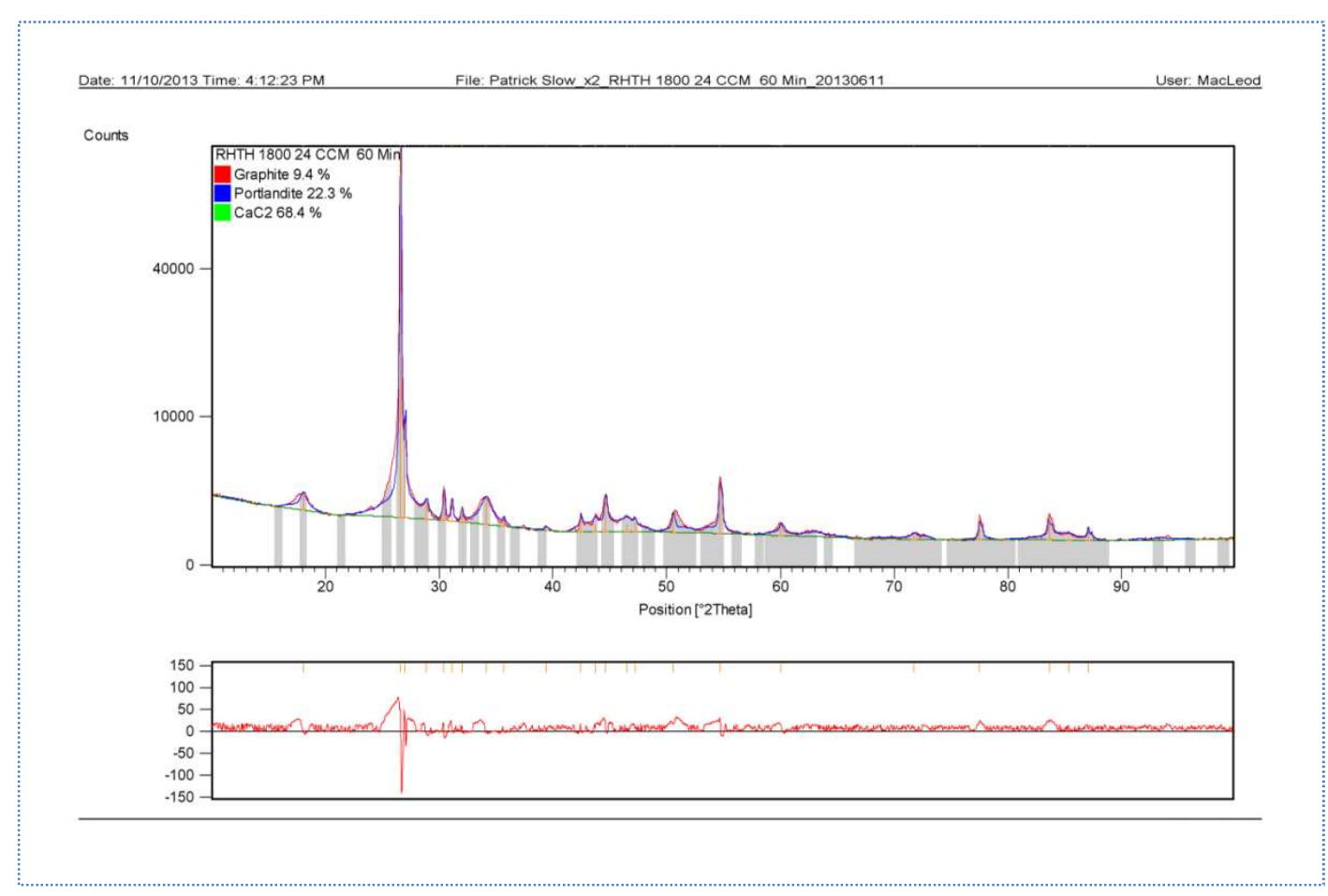

Figure 4.11: XRD of ball-milled lime and carbon (graphite) mixture after reaction- $1800^{\circ} \mathrm{C}$. Three $\mathrm{CaC}_{2}$ modifications identified. 

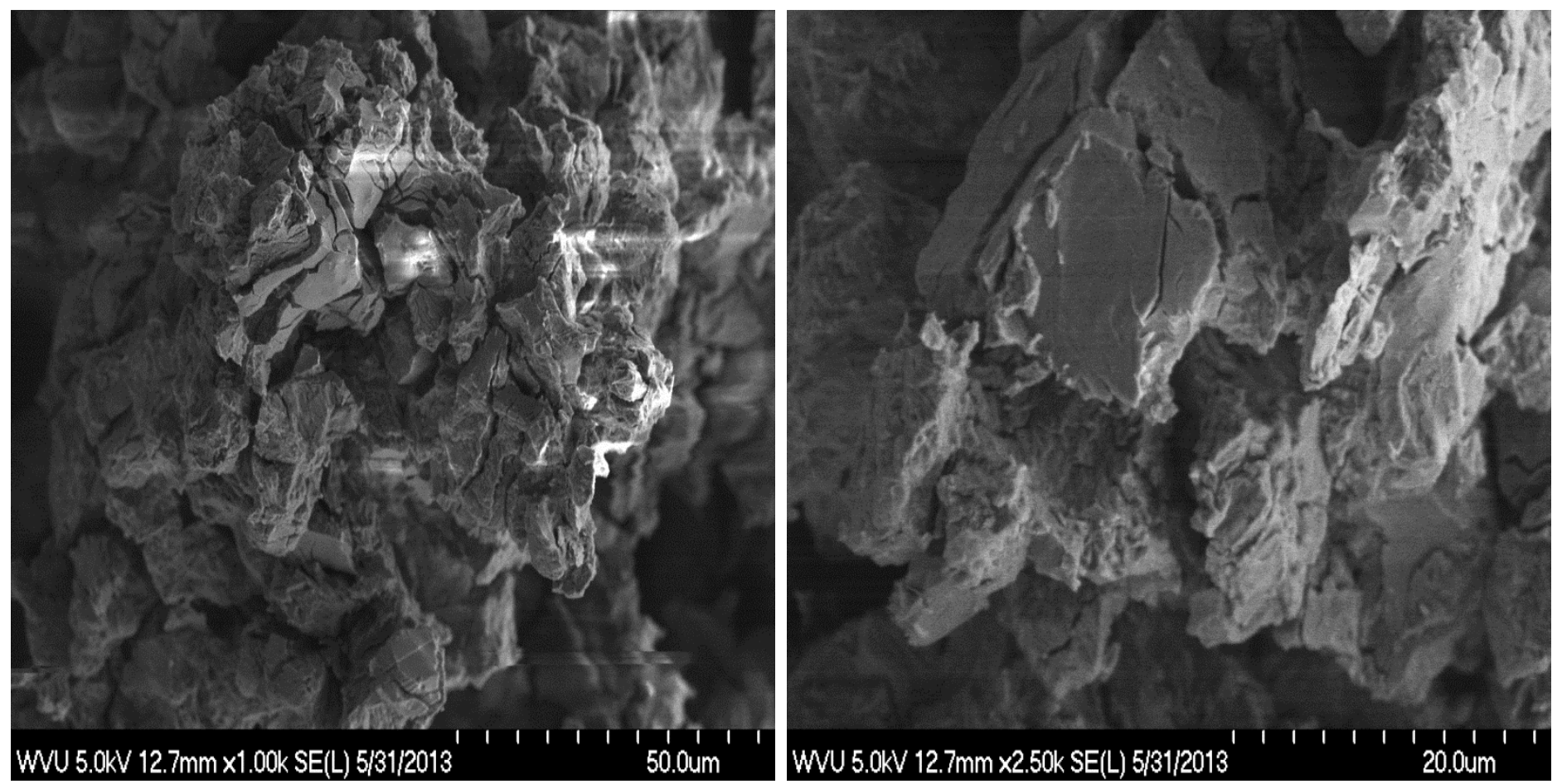

Figure 4.12: SEM of ball-milled lime and carbon (graphite) mixture after reaction @ $1800^{\circ} \mathrm{C}$.

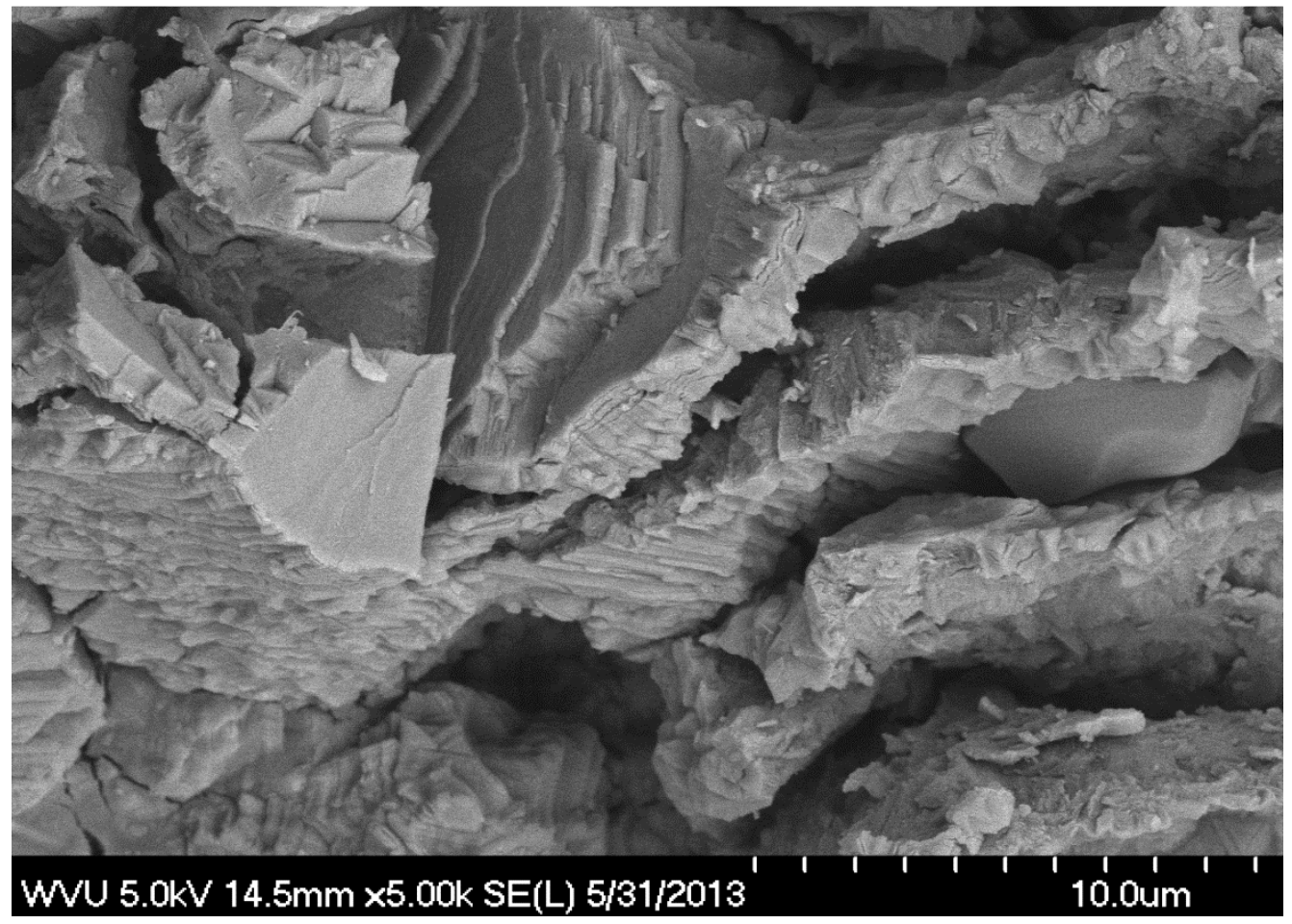

Figure 4.13: SEM of ball-milled lime and carbon (graphite) mixture after reaction @ $1700^{\circ} \mathrm{C}$. 


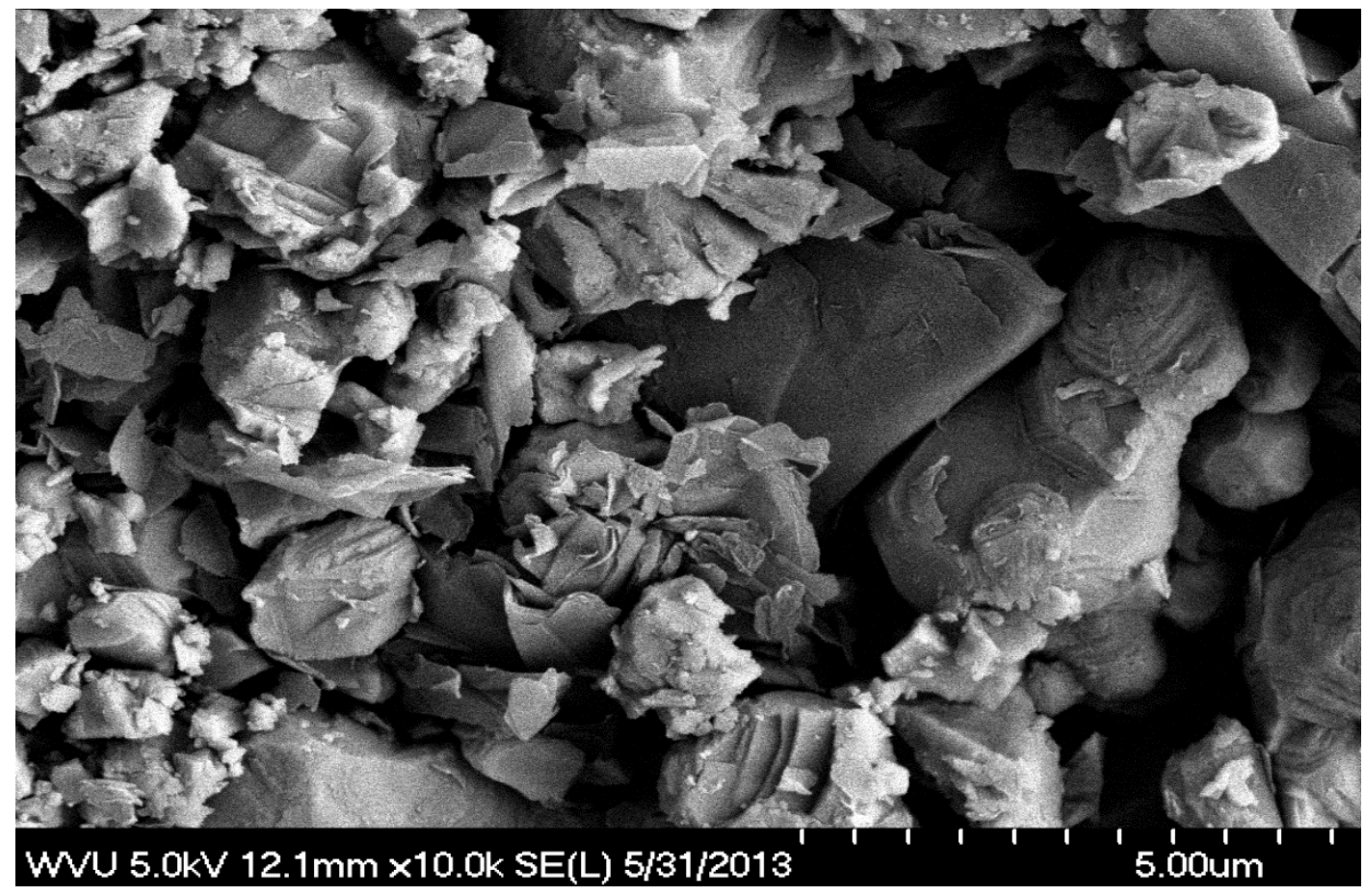

Figure 4.14: SEM of ball-milled lime and carbon (graphite) mixture after reaction @ $1600^{\circ} \mathrm{C}$.

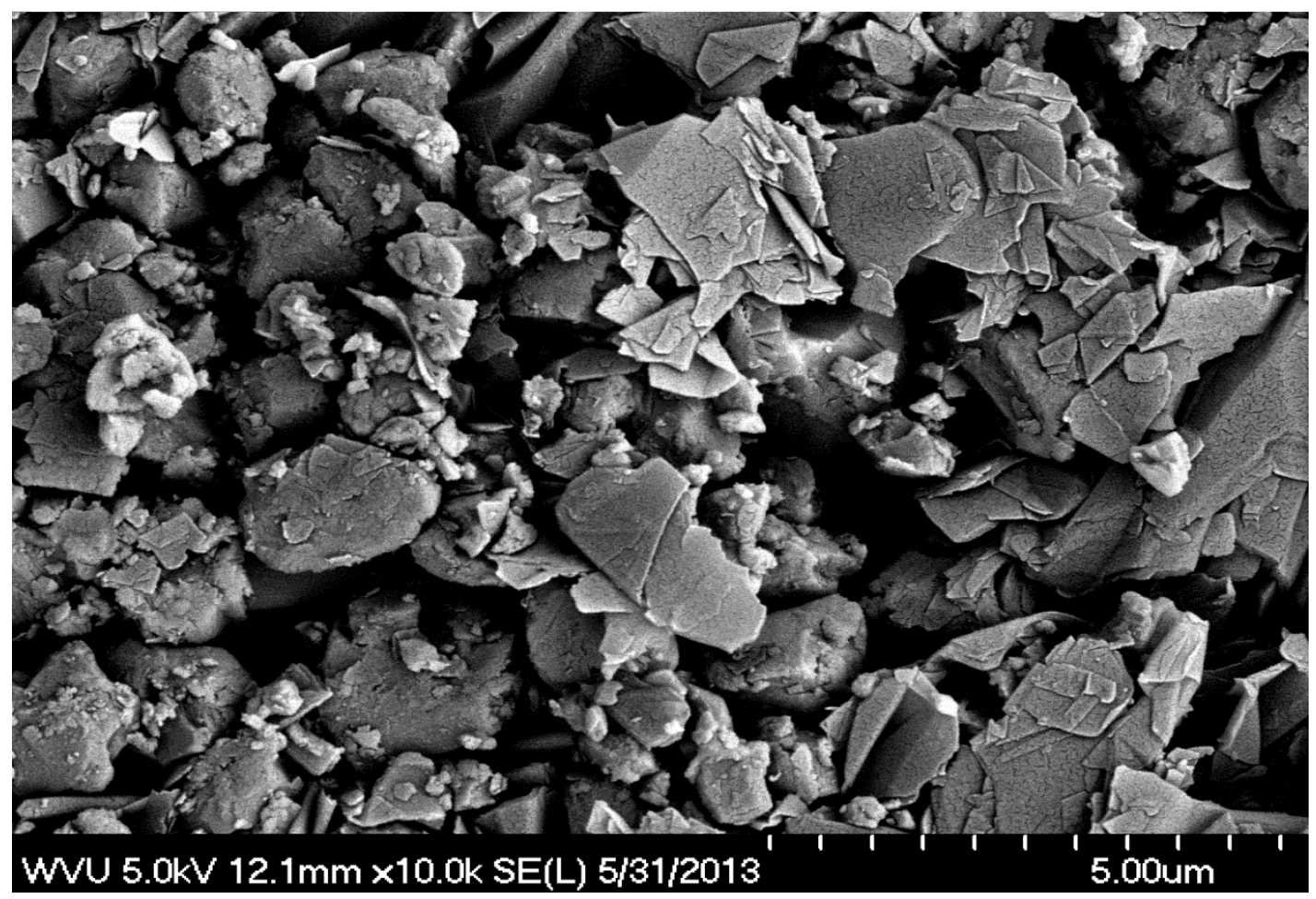

Figure 4.15: SEM of ball-milled lime and carbon (graphite) mixture after reaction @ $1500^{\circ} \mathrm{C}$. 
The reaction completed here seem to have proceeded differently in two temperature range: 1500 to $1640{ }^{\circ} \mathrm{C}$ and 1640 to $1700{ }^{\circ} \mathrm{C}$. The apparent activation energy calculated was found to be approximately $125.66 \mathrm{~kJ} \cdot \mathrm{mol}^{-1}$. The difference in activation energies when compared to that seen in literature might not be unconnected to the minimal $\mathrm{CO}$ pressure in this work as a result of constant purging by Argon gas, and that would yield much less activation energies. The local $\mathrm{CO}$ concentration may be low near the reaction interface, which would allow for the reaction to be completed closer to equilibrium conditions.

Another factor is the influence of the particle size used; the average particle in this work is about 9 times smaller than the starting $\mathrm{C} / \mathrm{CaO}$ materials reported in literature for calcium carbide formation.

The rate limiting factors considered are: 1) diffusion of $\mathrm{CaO}$ into $\mathrm{C}$ reactant, and 2) $\mathrm{CO}$ diffusion through the formed products. The literature understanding on the mass transfer limit for the reactants in terms of direction of the mass transfer, for $\mathrm{CaO}$ toward diffusion towards carbon or from carbon towards $\mathrm{CaO}$, or vice versa, has not been consistent [53]. Liu et al. showed that the mass transfer direction is mainly from $\mathrm{CaO}$ to carbon [53]. This is in agreement with the outcome of our experiments on how various carbon surface areas affect the initial temperature of the reaction. However, real time monitoring of this reaction in an environmental SEM will give a perfect answer to this, and we have it as part of the future works to be completed. 


\section{4: Chemical Reactions and Microwaves Heating}

\subsection{1: Temperature Measurement in Microwave Furnace}

A very important aspect of microwave experiments is the accurate measurement of temperature inside the furnace and this has been of serious concern in this work. There has been no generally agreed standard temperature measurement technique accepted in the microwave synthesis community. It is very important to note that the temperature measurement technique used should not interfere with the microwave field or disturb the thermal distribution of the sample being measured $[54,55]$. In this work, the temperature measurement in the microwave furnace was measured with a digital infrared pyrometer in this work. This is a Minolta-Land Cyclops 152 portable thermometer designed for temperature in the range 500 to $3200^{\circ} \mathrm{C}$. The digital output is made available over an RS-232 communication with a response time $30 \mathrm{~ms}$ (display $0.6 \mathrm{~s}$ ). The accuracy of the reading is $\pm 0.5 \%$. The pyrometer requires that the user manually enter an emissivity value for the specific sample being measured as well as manually focus on the sample through the hole on the lid of the insulation package. The distance from the sample top and the pyrometer gun tip should not exceed $1 \mathrm{~m}$. To ensure accuracy of temperature measured off the sample surface, the emissivity of the sample being tested was calibrated using a conventional furnace. Emissivity compensation is in range 0.1 to 1.0 .

The pellet samples were heated to temperatures from $1000^{\circ} \mathrm{C}$ to $1400^{\circ} \mathrm{C}$ and held for 30 minutes in a conventional furnace. The temperature for the calibration in the conventional furnace were measured with a K-type thermocouple and pyrometric shrinkage disks to determine the approximate emissivity values to be entered in the infrared pyrometer used during the reaction runs. A quartz window was employed in another identical set of experiments, to simulate the conditions during microwave sintering. 
Other conditions of the microwave were also simulated, including using the same sample container to hold the samples. Table 4.7 lists the measured emissivity, for the conditions of these calibration experiments in the conventional furnace, at various temperatures. The measured emissivity did not vary significantly with increasing temperature. The same values of emissivity were used for all microwave reaction experiments $(0.72 \pm 0.02$ at a $95 \%$ confidence interval with quartz window; $0.47 \pm 0.02$ at a $95 \%$ confidence interval without the quartz window). The accuracy of temperature measurement with the infrared pyrometer was determined to be within $5^{\circ} \mathrm{C}$ of the temperature measured with a K-type thermocouple in conventional sintering experiments.

Table 4.7: Infrared pyrometer calibration in conventional furnace for apparent emissivity measurements.

\begin{tabular}{|c|c|c|c|}
\hline \multicolumn{2}{|c|}{ Temperature $\left({ }^{0} \mathrm{C}\right)$} & Without window & With Window \\
\hline Thermocouple & TempChek & 0.70 & 0.46 \\
\hline 1400 & 1405 & 0.70 & 0.46 \\
\hline 1300 & 1398 & 0.71 & 0.46 \\
\hline 1200 & 1195 & 0.72 & 0.49 \\
\hline 1100 & 1104 & 0.75 & $0.47 \pm 002$ \\
\hline 1000 & 999 & $0.72 \pm 002$ & \\
\hline $\begin{array}{l}\text { Average values } \\
\text { (95\% confidence } \\
\text { measured range) }\end{array}$ & & & \\
\hline
\end{tabular}

\subsection{2: Microwaves Reactions}

The use of microwave energy has been attracting significant attention in recent years as an alternative method for processing ceramic materials. One of the most important reasons for this 
is the potential for reductions in manufacturing costs due to shorter processing times and consequent energy savings.

In this present work, we investigated the synthesis of calcium carbide in a multi-mode microwave furnace using a specific insulation package design (with a graphite crucible container to assist in rapid heat up of the reactants). The graphite crucible was used so that the reaction container would be capable to withstand possible shock while the material was processed in an inert atmosphere. Included with this work was a comparison between the reaction kinetics of the microwave processed material versus that of the conventional furnace. These experiments were conducted to verify the basic advantages of microwave application to carbo-thermic chemical reduction reactions with the expectation of developing a new kind of continuous reactor. The precursors used for this work are carbon graphite and calcium oxide materials in both pellet and powder forms. Carbon materials in powder form are very good absorbers of microwaves (easily heated by microwave radiation), and can heat other materials indirectly, or to act as a catalyst and microwave receptor in different heterogeneous reaction. Chen et al (1984) reported carbon to heat to $1000^{\circ} \mathrm{C}$ in $0.2 \mathrm{~min}$. Meanwhile, reagent grades calcium oxide, calcium carbonate, were reportedly classified as inactive, both reached $200^{\circ} \mathrm{C}$ and $130^{\circ} \mathrm{C}$ respectively at the rate of $5^{\circ} \mathrm{C} / \mathrm{min}$.

Even though calcium oxide is almost transparent to microwaves at room temperature, and hence cannot be heated readily, microwave energy will heat carbon powders almost instantaneously provided that the particle size is similar to the depth of penetration. This gives the possibility of heating homogeneous mixtures of lime and carbon from various sources extremely fast and volumetrically with higher energy conversion efficiency than can be achieved via conventional 
furnace heating. Microwave applications to chemical reactions open new possibilities: fast and efficient means of processing ceramic carbide powders; however, special care needs to be taken over the selection of the ceramic system and the reaction environment.

In the experiments completed, the microwave furnace was used to heat the samples to temperatures between 1000 and $1600^{\circ} \mathrm{C}$, as measured by the pyrometer strategically placed to measure the sample surface temperatures. Once the desired temperature was obtained, the power to the furnace was shut off and the samples were allowed to cool rapidly. Similar to conventional tube furnace outcome, the samples were analyzed for weight loss and the amount of calcium carbide present.

In figure 4.16, the effect of holding time and temperature on $\mathrm{CaC}_{2}$ formation for microwave heating were shown. The curve is approximately exponential which suggests a diffusioncontrolled path. Part of the future works for these experiments involved 10 minutes time interval study of reaction of $1 / 3$ mixture of $\mathrm{C} / \mathrm{CaO}$, to understand the actual reaction mechanism.

Figure 4.17 compares the calcium carbide yields obtained in the microwave furnace to those obtained in the tube furnace experiments. Samples were analyzed with the GC to quantify the amount of carbide formed and XRD to identify phases present in the final product. Calculations and data are presented in appendix I. The reacted samples were pre- getting rid of the hydroxide inclusion that erroneously contributes to weight loss usually reported. As presented here, the microwave furnace process leads to significantly greater yields, with higher reaction temperature or longer holding times yielding more $\mathrm{CaC}_{2}$. The weight loss reported here includes both $\mathrm{Ca}$ loss, hydroxide inclusion and the decomposition of formed $\mathrm{CaC}_{2}$. 


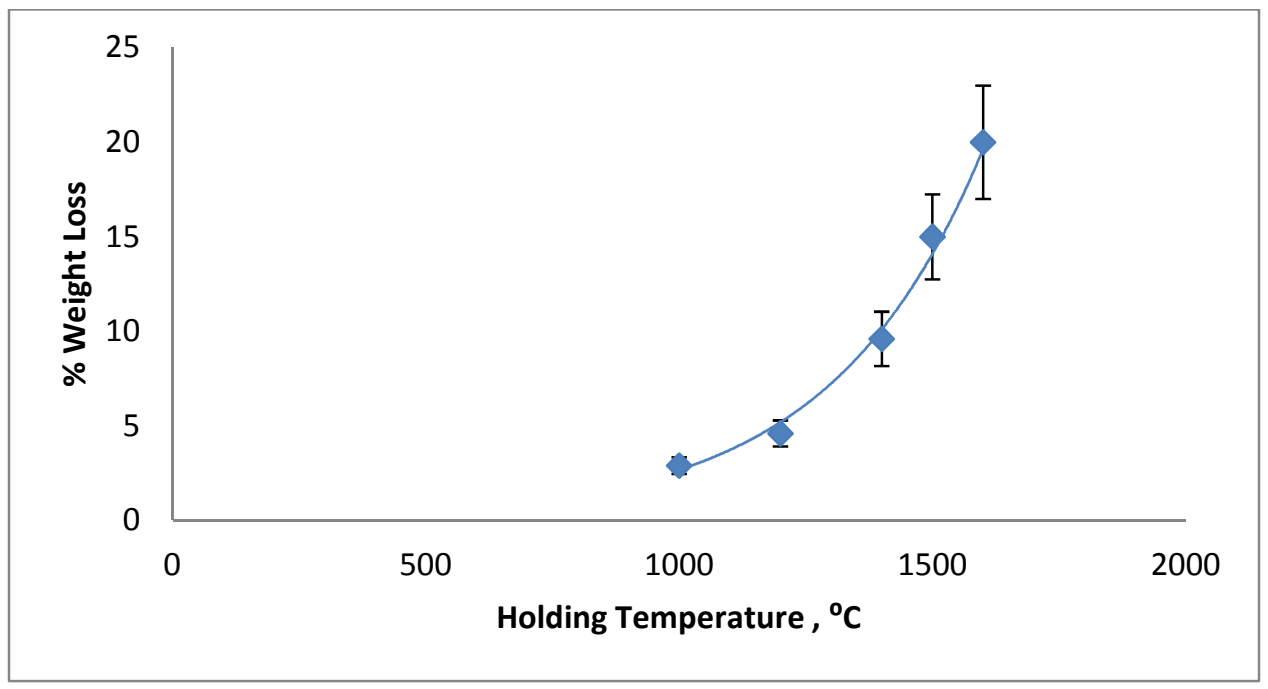

Figure 4.16: Percent weight loss as a function of maximum microwave furnace temperature. $\mathrm{C} / \mathrm{CaO}$ mixture average particle size of approximately $15 \mu \mathrm{m}$.

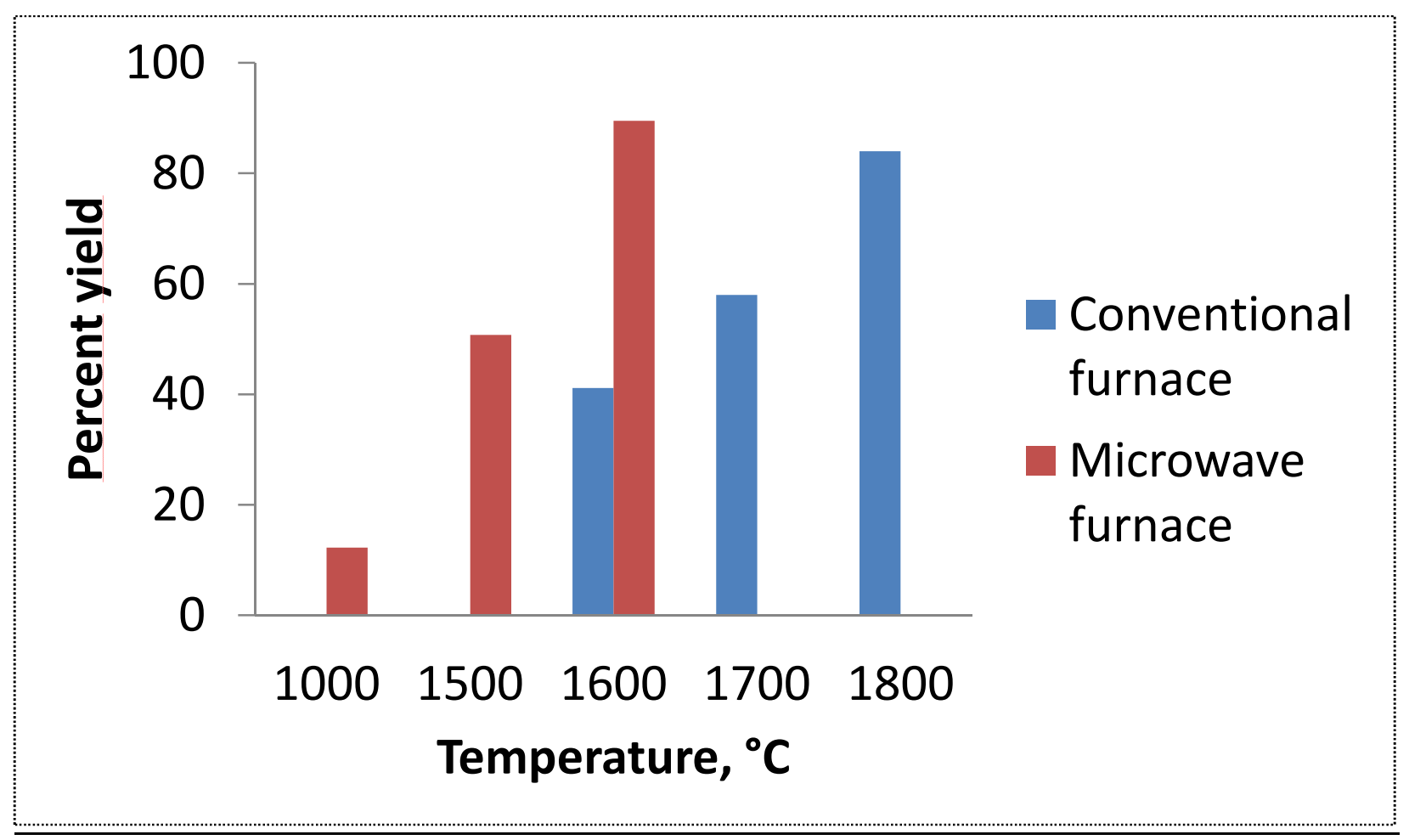

Figure 4.17: Comparison of calcium carbide yields in conventional tube and microwave furnaces. $\mathrm{C} / \mathrm{CaO}$ mixture average particle size of approximately $15 \mu \mathrm{m}$.

It is clear that temperature has a profound influence on the rate of the reaction. Unlike in the conventional heating whereby calcium vaporization and subsequent loss seem to be increased, it 
is however not the case with microwave. Calcium oxide consumption (estimated from both weight loss and XRD) increased fairly exponentially as temperature increased (as shown in figure 4.18). The mode of heating basically influenced the reaction rate significantly, where the intrinsic heating due to microwave coupling resulted in high reaction rates locally (which are different from that of conventional heating).

A significant feature to note in figure 4.18 , which shows the amount of calcium oxide reduced for different temperature levels and time, is the initiation of carbo-thermal reduction at temperatures much lower than expected. From the data obtained, reduction commenced at $1000^{\circ} \mathrm{C}$ for reaction completed inside the microwave furnace. The lowest temperature for carbide formation reported for conventional methods is at $1460^{\circ} \mathrm{C}$ [53]. The use of microwave heating clearly resulted in apparent enhancement in the chemical reaction as expected.

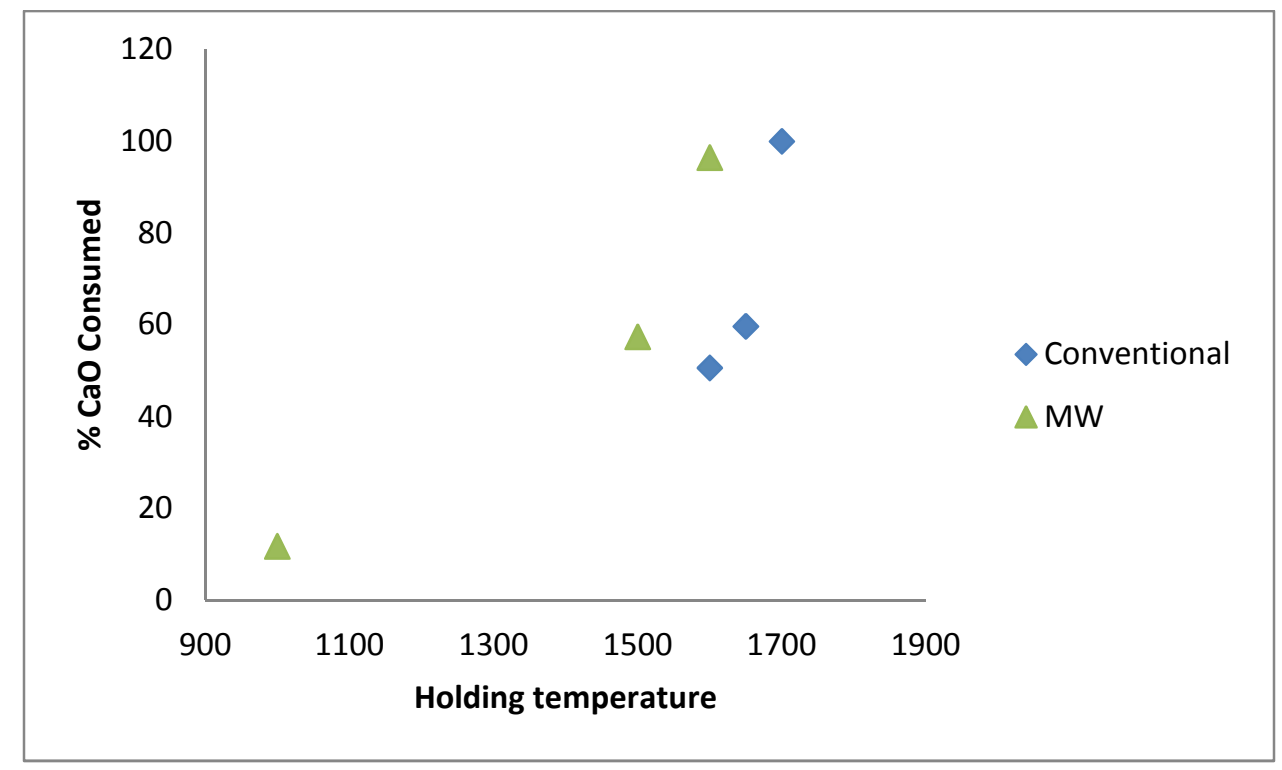

Figure 4.18: Comparison of calcium oxide consumed via microwave (MW) and conventional heating. 
The use of some materials as subsectors (preheaters) during microwave heating depends on the response rate of the material in absorbing microwave energy; this is especially useful to aid increase in heating rate and sintering uniformity for ceramic insulators. These susceptor materials are usually in coarse powder sand but they could actually be in solid block as well, for example a rod like shape, for strategic placement in preferred position inside the heating package. High dielectric loss materials such as silicon carbide with a high response rate are most times used as sintering bed or packaging material for a ceramic insulator that only absorbs microwave energy at elevated temperature. The configuration and arrangement of these susceptors to be used per specific ceramic powder are important factors.

In our work, $\mathrm{C} / \mathrm{CaO}$ mixture was placed inside a graphite crucible enclosed in an alumina insulating casket. We did not have the traditional $\mathrm{SiC}$ or other qualified packing materials as susceptor; the use of graphite as part of the reacting mixture and as crucible seem to have served as microwave absorption platform. The graphite crucible as used act as both container vessel and the preheater in this case. The casket design is simple in design with the primary aim to minimize heat loss and also homogenized heat distribution around the heating environment in the course of the reaction. Another important thing to note in our work is the system of filling and purging the reaction environment as described in the experimental section here. Presence of oxygen could combine with $\mathrm{CO}$ to form $\mathrm{CO}_{2}$ and this further combine with $\mathrm{Ca}(\mathrm{OH})_{2}$ from $\mathrm{CaC} 2$ interaction with air moisture in the process of cooling the furnace to form calcium carbonate as the final or part of the final product . 


\subsection{Conclusion}

Calcium carbide with high purity and density compared to conventional technique was prepared via microwave heating in which lime and carbon were reacted in a graphite cup at higher temperature in an argon environment. Table 5.1 summarize some of the advantages observe from the preliminary experiments performed. The total cycle time was cut by $80 \%$ in the microwave over conventional approach. It is important to mention that the heating rate used in the conventional method was constant at $3^{0} / \mathrm{min}$. while that of the microwave varied from 1$45^{\circ} / \mathrm{min}$. However, when the conventional heating rate was set at $5^{\circ} / \mathrm{min}$., weight loss dropped by approximately $32 \%$ in the conventional reactions completed at $1600^{\circ} \mathrm{C}, 60 \mathrm{~min}$. This thus suggests that the rate of heating does make a difference.

Major challenges experienced while setting up this process were selection of the right insulation package, heating vessel and also controlling random hot spot melting during microwave heating. To ensure accurate recording of temperature for microwave heating, infrared pyrometer was employed in the microwave furnace to read temperature and was calibrated against a conventionally heated furnace.

Table 5.1: Comparison of microwave and conventional carbo-thermic reduction of lime reactions completed.

\begin{tabular}{lcc}
\hline & Microwave & Conventional \\
\hline Reaction Temperature $\left({ }^{0} \mathrm{C}\right)$ & 1600 & 1800 \\
Total Cycle Time (Minutes) & 135 & 1200 \\
Hold time (Minutes) & $0-60$ & $0-60$ \\
\% Conversion & $80-90.99$ & $75-85$ \\
\hline
\end{tabular}


The residual materials after the reactions were calcium oxide, unreacted calcium and free carbon. The types of reaction container condition, insulation package, particle size, pellet compaction load and reaction time and temperatures influenced the products purity and densification.

Overall, the results of this work showed that microwave heating could be used to synthesize $\mathrm{CaC}_{2}$ uniformly and quickly at lower temperatures than conventional method. However, contamination of samples caused by hydrolysis of the highly reactive $\mathrm{CaC}_{2}$ samples formed to atmospheric air, during ejection and transfer into the glove box for further processing, should be eliminated completely. In addition, pre-reaction contamination such as $\mathrm{CaO}$ conversion into $\mathrm{Ca}(\mathrm{OH})_{2}$ could also contribute about $25 \%$ of $\mathrm{H}_{2} \mathrm{O}$ ( amount contribution depends on the level of exposure) picked up during ball-milling and sieving after ball-milling of samples. In our experiments, these contaminations were limited (completely removed before reaction) by introducing additional heat-treatment step before introduction of sample into the furnace and the use of air-tight sample holders during x-ray analyses.

The problems described above were fixed and similar experiments were completed using conventional furnace. The reactions completed in conventional furnaces where fitted in a 3dimensional: Degree of reaction $(=$ apparent conversion $)=(1-\sqrt[3]{(1}-\alpha))^{2}=k t_{\text {react }}$. Here we found the activation energy to be $107 \mathrm{~kJ} / \mathrm{mol}$ and $12 \mathrm{~kJ} / \mathrm{mol}$ for lower and higher temperature range respectively, which was different from what was found in literature. Also we found the apparent reaction kinetic constants at $1500^{\circ} \mathrm{C}, 1600^{\circ} \mathrm{C}$ and $1700^{\circ} \mathrm{C}$ temperature levels as 0.0001 , 0.0041 and 0.006 respectively per minute.

An analytical independent determination of all major constituents of the products after reaction was developed. Rietveld technique as used determined the mass composition for all phases 
present in simple steps, compare to several multi-steps chemical titration and back calculation and estimation and exit $\mathrm{CO}$ monitoring that exclude trapped amount as practiced for decades. The standard deviation was $5 \%$. 


\subsection{Future Work}

This work mainly focused on gathering experimental kinetic data and measures the conversion rates for reaction completed in both conventional tube furnace and microwave furnace. While we have been able to characterize the reaction rates of carbon and calcium oxide precursors with various size ratios and chemistries and also draw a correlation between the particle characteristics and the kinetic constants obtained from the reactions completed, there is also need to perform these experiments in detail.

1) Using other carbon sources such as coal, carbon black and coal char to measure their influence using relatively smaller sizes than what is presently found in the literature. The reactivity of carbon does affect the formation of carbide as we have shown in terms of weight loss at $1500^{\circ} \mathrm{C}, 60$ minutes. It is important to first effectively quantify through a proximate analyses, the amount of carbon content in coal for instance, and the role other volatile materials present play, so as to be able to compare the outcome with carbon graphite. This will be different from what others have done in the past since this reaction will be completed inside the microwave and phase quantification by XRD Rietveld technique. Complete coal and coal-char based reactions at $1500^{\circ} \mathrm{C}-1700^{\circ} \mathrm{C}$ with slow and fast cooling reaction process for both powder and pellet samples. Variables are packing density, morphology, reaction time and thickness.

2) The role of smaller calcium oxide sizes specifically to determine how it influences solid-solid surface contact and mass transfer at that level. The inability to effectively quantify the amount that vaporizes is reasonable for conflicting information on kinetic mechanism of this reaction from literature. 
3) In situ monitoring of the influence of $\mathrm{CO} / \mathrm{Ar}$ mix composition variation on the reaction mechanism.

4) Continue tests in the microwave furnace for enhanced transformation kinetics and low power consumption; completing at 15 minutes holding time interval to determine the reaction rates constant and activation energy $\left(1000-2100^{\circ} \mathrm{C}\right)$.

5) Initiate exploratory real-time reaction monitoring of morphological influence on the reaction mechanism using e-SEM. 


\section{Appendix I}

\section{$\underline{\text { Sample of Calculation }}$}

Calculation is based on the hydrolysis of calcium carbide according to reaction I:

$$
\mathrm{CaC}_{2}(\mathrm{~s})+2 \mathrm{H}_{2} \mathrm{O}(\mathrm{l})<=\mathrm{C}_{2} \mathrm{H}_{2}(\mathrm{~g})+\mathrm{Ca}(\mathrm{OH})_{2}(\mathrm{~s})
$$

1. Amount of acetylene dissolved in solution

Temperature of reaction $=298^{\circ} \mathrm{K}$

Pressure of the system $=0.52 \mathrm{~atm}$

Henry's constant, $\mathrm{H}=1.28 \times 10^{\wedge} 3$

Since $\mathrm{p}=$ H.X,

Mole of $\mathrm{C}_{2} \mathrm{H}_{2}$ dissolved per mole solution $=\frac{0.52}{1.28 \times 10^{3}}$

$$
=0.00040625
$$

Therefore, $\mathrm{C}_{2} \mathrm{H}_{2}$ dissolved in solution $=(0.00040625)\left(\frac{125}{18}\right)$

$$
=0.00282 \text { moles }
$$

2. Calcium carbide estimation

Volume of system $=$ volume of cylinder + volume of flask + volume of pipe connetion

$$
=630 \mathrm{ml}
$$

Gas generated $=\frac{(0.52)(630)}{(82.06)(298)}=0.0134$ moles 
Moles of $\mathrm{C}_{2} \mathrm{H}_{2}$ from the composition obtained from the $\mathrm{GC}=0.0134(0.05)$

Total moles of $\mathrm{C} 2 \mathrm{H} 2=0.0035$

Weight percent of $\mathrm{CaC} 2=(0.0035)(64.08)=0.2236 \mathrm{~g}$

Yield percent $=0.2236 \mathrm{~g} / 0.5555 \mathrm{~g}=40.25$ 


\section{References}

1. http://www.airproducts.com/ /media/Files/PDF/company/safetygram-13.pdf, page 2 Air Products and Chemicals, Inc., 1994

2. P. Schwarzkopf and R. Kieffer, Refractory Hard Metals-Borides, Carbides, Nitrides and Silicides. Mc Millan Co.,NY (1953).

3. M. H. El-Naas, R. J. Munz and F. Ajersch, Solid-Phase Synthesis of Calcium Carbide in a Plasma Reactor, Plasma Chemistry and Plasma Processing Volume 18, Number 3 (1998), 409427.

4. K. Baba and N. Shohata, Manufacture of calcium carbide micropowders by high frequency plasma method, Japanese patent, No. 63112409 (1988).

5. P. Hellmold and $\mathrm{W}$. Gordziel, Investigation of the particular reactions of $\mathrm{CaC}_{2}$ : Investigation of the formation of $\mathrm{CaC}_{2}$ from calcium oxide and carbon, Chem. Technol. 35, 297-300 (1983).

6. Mu, J. Jacob, and Robert A. Hard, A rotary kiln process for making calcium carbide. Industrial \& engineering chemistry research 26.10 (1987): 2063-2069.

7. C. Brookes, C. E. Gall, and R. R. Hudgins, A model for the formation of calcium carbide in solid pellets, Can. J. Chem. Eng. 53, 527-535 (1975).

8. H. Tagawa and H. Sugawara, The kinetics of the formation of calcium carbide in solid-solid reaction, Bull. Chem. Soc. Jpn. 35, 1276-1279 (1962).

9. C.W. Zhu, G. Y. Zhao and V. Hlavacek, A de plasma-fluidized bed reactor for the production of calcium carbide. Journal of materials science 30.9 (1995): 2412-2419.

10. Guodong Li, Qingya Liu, Zhenyu Liu, Z. Conrad Zhang, Chengyue Li, and Weize Wu, Production of Calcium Carbide from Fine Biochars, Angew. Chem. Int. Ed. 2010, 49, 84808483.

11. N. A. Hassine, J. G. Binner and P. Cross, Synthesis of Refractory Metal Carbide Powders via Microwave Carbothermal Reduction, T. E. International Journal of Refractory Metals and Hard Materials vol. 13 issue 6, 1995 p. 353-358.

12. D. Agrawal, Latest developments in microwave materials processing, Materials research innovations, 14 (1) (2010) 3-8.

13. E.T. Thostenson and T. W. Chou, Microwave processing: fundamentals and applications Composites Part A: Applied Science and Manufacturing, 30 (9), pp. 1055-1071(1999).

14. Craig Saltiel, Heat and mass transfer in microwave processing, Advances in heat transfer, 33, (1999) 1-94. 
15. Chris Y. Fang \& Clive A. Randal \& Michael T. Lanagan \& Dinesh K. Agrawal, Microwave processing of electroceramic materials and devices, J Electroceram 22:125-130 (2009).

16. M. Oghbaei, O. Mirzaee, Microwave versus conventional sintering: A review of fundamentals, advantages and applications, Journal of Alloys and Compounds, 494 (2010) 175 189.

17. T. Mazona, M.A. Zaghete, J.A. Varela and E. Longo, Barium strontium titanate nanocrystalline thin films prepared by soft chemical method, Journal of the European Ceramic Society 27 (2007) 3799-3802.

18. W.H. Sutton, Microwave processing of ceramic materials, Ceramic Bulletin; 68(2): (1989) $376-384$.

19. M. A Janney, C.L.Calhoun and H.D.Kimrey, Microwave Sintering of Solid Oxide Fuel Cells- 1.Ziconia -8\% Yttria, J. Am. Ceram. Soc., 75(2), (1992) 341-346.

20. P.D.Ramesh, D.G.Brandon, L. Schachter, Use of partially oxidized SiC particle bed for microwave sintering of low loss ceramics, Mater. Sci. Engg. A., 266 (1-2), (1992) 211-220.

21. Guanghua Liu, Jiangtao Li and Kexin Chen, Combustion Synthesis of Ceramic Powders with Controlled Grain Morphologies, Advances in Ceramics - Synthesis and Characterization, Processing and Specific Applications, Prof. Costas Sikalidis (Ed.), ISBN: 978-953-307-505-1, InTech, (2011). Available at: http://www.intechopen.com/books/advances-in-ceramics-synthesisand-characterization-processing-and-specific-applications/combustion-synthesis-of-ceramicpowders-with-controlled-grain-morphologies.

22. Yasuo Gotoha, Kensaku Fujimuraa, Mikio Koikea, Yutaka Ohkoshia, Masanobu Naguraa, Kensuke Akamatsub and Shigehito Dekib, Synthesis of titanium carbide from a composite of TiO2 nanoparticles/methyl cellulose by carbothermal reduction, Materials Research Bulletin 36 (2001) 2263-2275.

23. C. N. Lin and S. L. Chung, Combustion synthesis method for synthesis of aluminum nitride powder using aluminum containers (II). J. Mater. Res., 19(3), (2004) 3037-3045.

24. http://www.chemsystems.com/reports/search/docs/prospectus/MC06_Acetylene-Pros.pdf

25. F. H. McRitchie and N. N. Ault, Design of a High-Temperature Resistance Furnace. Journal of the American Ceramic Society, (1950), 33: 25-26.

26. http://www.agni.de/chinese/2100C_furnace.htm.

27. Lazau, Peculiarities of ceramics powder synthesis using combustion method, Revue Roumaine de Chimie, 2005, 50(11-12), 919-927.

28. Young-Chul Woo, Ho-Jae Kang and Deug J. Kim, Formation of TiC particle during carbothermal reduction of $\mathrm{TiO}_{2}$, Journal of the European Ceramic Society 27 (2007) 719-722. 
29. M. B. Muller, Structure, properties and reactions of $\mathrm{CaO}$ in burnt lime, Scand. J. Metall. 1990, 19, 64-71. Part 1-3.

30. H.S. Hao, L.H. Xu, Y. Huang, X.M. Zhang, Z.P. Xie, Kinetics mechanism of microwave sintering in ceramic materials, Science in China Series E: Technological Sciences, 52, (2009) $2727-2731$.

31. S. L. McGilla, J. W. Walkiewicza and G. A. Smyresa, The effects of power level on the microwave heating of selected chemicals and minerals, Reno Research, Bureau of Mines, pp. (1988) 247-252.

32. Cheng-Yu Hsieh, Chun-Nan Lin, Shyan-Lung Chung, Jiping Cheng, Dinesh K. Agrawal, Microwave sintering of AIN powder synthesized by a SHS method, Journal of the European Ceramic Society 27 (2007) 343-350.

33. D. M. P. Mingos, D. R. Baghurst, Chem. Soc. Rev. 1991, 20, 1.

34. Johanna Nylén, Sumit Konar, Peter Lazor, Daryn Benson and Ulrich Häussermann1, Structural behavior of the acetylide carbides $\mathrm{Li}_{2} \mathrm{C}_{2}$ and $\mathrm{CaC}_{2}$ at high pressure, The Journal of Chemical Physics 137, 224507 (2012).

35. http://www.atlantachemical.com/learning_center/microwave_heating.htm

36. G. Li, Q. Liu, Z. Liu, Zhang, Z. C., C. Li and W. Wu, Production of Calcium Carbide from Fine Biochars, Chem. Int. Ed., 49: (2010), 8480-8483.

37. N. Makaratat, C. Jaturapitakkul, and T. Laosamathikul, Effects of Calcium Carbide ResidueFly Ash Binder on Mechanical Properties of Concrete. J. Mater. Civ. Eng., 22(11), (2010), 11641170 .

38. L.G. Carreiro et al., Fuel processing technology 91 (2010), 1028-1032.

39. Guodong Li, Qingya Liu, Zhenyu Liu, Z. Conrad Zhang, Chengyue Li, and Weize Wu, Production of calcium carbide from Fine Biochars, Angew. Chem. Int. Ed. (2010) 49, 84808483.

40. J.A. Menéndez, A. Arenillas, B. Fidalgo, Y. Fernández, L. Zubizarreta, E.G. Calvo and J.M. Bermúdez, Microwave heating processes involving carbon materials, Fuel Processing Technology 91 (2010) 1-8.

41. T.T. Chen, J.E. Dutrizac, K.E. Haque, W. Wyslouzil and S. Kashyap, The relative transparency of minerals to microwave radiation, Can. Metall. Quart. 23 (1), 1984: 349-351.

42. N. Kameyama, Electrochemistry: Theory and Application, Vol. III-2, 1956, pp. 134-141.

43. Mukaibo and Yamanaka, Kinetics of the first stage of the reaction proceeding calcium carbide under reduced pressure, J. Chem. Soc. Japan, Ind. Chem. Sect. 56, 1953: pp. 73-75. 
44. M. Knapp and U. Ruschewitz, Structural Phase Transitions in $\mathrm{CaC}_{2}$, Chem. Eur. J. $2001,7,874$.

45. Ruth Ahlers and U. Ruschewitz, On the Synthesis of Binary and Ternary Carbides in a Modified Domestic Microwave Oven, Journal of Inorganic and General Chemistry, Volume 631, Issue 6-7, May 2005: pages 1241-1246.

46. G. A. Meerson, Yu. G. Olesov and V. I. Pryalochnikov, The reduction of zirconium dioxide by calcium carbide and cyanamide, Soviet Atomic Energy Volume 14, Issue 6, 1963 ,pp 586592.

47. Shi, Dejun, Ke Qiao, and Zifeng Yan. Effect of potassium carbonate on catalytic synthesis of calcium carbide at moderate temperature. Frontiers of Chemical Science and Engineering 5.3 (2011): 372-375.

48. http://www.cem.com/page130.html

49. R. Gedye, F. Smith and K. Westerway, The use of microwave ovens for rapid organic synthesis, Tetrahedron letters 1986, 27(3); 279-282.

50. T. Wang, R. Y. Liu, M. L. Zhu and J. S. Zhang, Activation Energy of Self-Heating Process Studied by DSC, Journal of Thermal Analysis and Calorimetry, vol. 70, pp. 507-519, 2002.

51. Chun Lin He, Shao Jian Ma, Xiu Juan Su, Yan Qing Chen and Yu Shi Liang, Calorimetry Study of Microwave Absorption of Some Solid Materials, Journal of Microwave Power and Electromagnetic Energy, 47 (4), 2013, pp. 251-261.

52. G. $\mathrm{Li}$; Q. Liu and Z. $\mathrm{Liu}, \mathrm{CaC}_{2}$ production from pulverized coke and $\mathrm{CaO}$ at low temperatures reaction mechanisms, Ind. Eng. Chem. Res. 2012, 51, 10742-10747.

53. G. Li; Q. Liu and Z. $\mathrm{Liu}, \mathrm{CaC}_{2}$ production from pulverized coke and $\mathrm{CaO}$ at low temperatures influence of minerals in coal-derived coke, Ind. Eng. Chem. Res. 2013, 51, 1074810754.

54. E. Pert, Y. Carmel, A. Birnboim, T. Olorunyolemi, D. Gershon, J. Calame, I.K. Lloyd, and O.C. Wilson, Jr., Temperature Measurements during Microwave Processing: The Significance of Thermocouple Effects, J. Amer. Ceram. Soc., 84 [9] 1981-1986 (2001).

55. R. W. Bruce, A. W. Fliflet, R. P. Fischer, D. Lewis, III, B. A. Bender, G.M. Chow, R. J. Rayne, L. K. Kurihara, and P. E. Shoen, Millimeter-Wave Processing of Alumina Compacts, pp 287-294 in Ceramic Transactions, Vol. 80, Microwave: Theory and Application in Materials Processing IV. Edited by D. E. Clark, W. H. Sutton and D. A Lewis. American Ceramic Society Inc., Westerville, OH, 1997. 
56. Ammar Khawam, and Douglas R. Flanagan, Solid-State Kinetic Models: Basics and Mathematical Fundamentals, J. Phys. Chem. B, 2006, 110 (35), 17315-17328

57. Hahne, B, Gordziel, and W, Meerbote (1990), Some results of microscopic and x-rayinvestigations of calcium carbide, Crystal Research and Technology, 25(3), 313-324.

58. Steven Lee Rowan, Ismail Celik, Jose Escobar Vargas, Suryanarayana Raju Pakalapati, and Matt Targett, Reaction Kinetics Modeling of $\mathrm{CaC}_{2}$ Formation From Coal and Lime, Ind. Eng. Chem. Res., Just Accepted Manuscript : Publication Date (Web): 16 Jan 2014. 
